\title{
Weak decays of triply heavy baryons
}

\author{
Wei Wang and Ji Xu* \\ INPAC, Shanghai Key Laboratory for Particle Physics and Cosmology, \\ MOE Key Laboratory for Particle Physics, Astrophysics and Cosmology, \\ School of Physics and Astronomy, Shanghai Jiao Tong University, \\ Shanghai 200240, China
}

(Received 10 March 2018; published 25 May 2018)

\begin{abstract}
After the experimental establishment of doubly heavy baryons, baryons with three quarks are the last missing pieces of the lowest-lying baryon multiplets in the quark model. In this work, we study semileptonic and nonleptonic weak decays of triply heavy baryons, $\Omega_{c c c}^{++}, \Omega_{c c b}^{+}, \Omega_{c b b}^{0}$, and $\Omega_{b b b}^{-}$. Decay amplitudes for various channels are parametrized in terms of a few SU(3) irreducible amplitudes. We point out that branching fractions for Cabibbo-allowed processes, $\Omega_{c c c}^{++} \rightarrow\left(\Xi_{c c}^{++} \bar{K}^{0}, \Xi_{c c}^{++} K^{-} \pi^{+}, \Omega_{c c}^{+} \pi^{+}, \Xi_{c}^{+} D^{+}\right.$, $\left.\Xi_{c}^{\prime} D^{+}, \Lambda_{c} D^{+} \bar{K}^{0}, \Xi_{c}^{+} D^{0} \pi^{+}, \Xi_{c}^{0} D^{+} \pi^{+}\right)$, may reach a few percent. We suggest our experimental colleagues to perform a search at hadron colliders and the electron and positron collisions in the future, which will presumably lead to discoveries of triply heavy baryons and complete the baryon multiplets. Using the expanded amplitudes, we derive a number of relations for the partial widths that can be examined in the future.
\end{abstract}

DOI: $10.1103 /$ PhysRevD.97.093007

\section{INTRODUCTION}

In the past decades, hadron spectroscopy has experienced a rapid renaissance, predominantly propelled by discoveries of a number of hadron resonances that defy the standard quark model interpretations. These resonant states are generically classified as hadron exotics, and for reviews on recent progress, please see Refs. [1-4]. Among all exotic hadrons, $X(3872)$ plays a most important role due to its special properties. Aside from these unexpected discoveries, there has also been gradual progress in the traditional sector of the charmonium and bottomonium spectroscopy. One of the highlights in recent years is the discovery of $\Xi_{c c}^{++}$by the LHCb Collaboration [5]:

$$
m_{\Xi_{c c}^{++}}=(3621.40 \pm 0.72 \pm 0.27 \pm 0.14) \mathrm{MeV} .
$$

This observed state $\Xi_{c c}^{++}$fills well in the quark model as the lowest-lying $c c u$ baryon [6]. After the tentative establishment of $\Xi_{c c}^{++}$, it is plausible to fill the baryon family with the last missing members, namely, baryons made of three heavy quarks. Charm and bottom quarks are much heavier than the $u, d$, and $s$; thus, baryons with three heavy quarks will refrain from light-quark contaminations, and the study

\footnotetext{
*xuji1991@sjtu.edu.cn
}

Published by the American Physical Society under the terms of the Creative Commons Attribution 4.0 International license. Further distribution of this work must maintain attribution to the author(s) and the published article's title, journal citation, and DOI. Funded by SCOAP ${ }^{3}$. of triply heavy baryons can help us better understand the dynamics of strong interactions.

Previous studies of triply heavy baryons concentrated on three facets: spectroscopy, production, and decays. Most theoretical investigations in the literature, especially in recent years, have focused on the masses and magnetic moments [7-20], while less attention has been paid to the production and decay properties. The only available estimate of the production is conducted in Refs. [21,22], where the cross sections at the LHC with $\sqrt{s}=7 \mathrm{TeV}$ are found to reach the $0.1 \mathrm{nb}$ level depending on different kinematics cuts. In the $b \rightarrow c$ transitions among triply heavy baryons, Ref. [23] has discussed the implications of heavy-quark spin symmetry. Some decay modes of the $\Omega_{c c c}^{++}$were analyzed for the first time in Ref. [24].

The main focus of this paper is to provide a systematic analysis of weak decays of the lowest-lying triply heavy baryons, $\Omega_{c c c, c c b, c b b, b b b}$. The $\Omega_{c c c}$ and $\Omega_{b b b}$ have spin 3/2, while the $\Omega_{c c b}$ and $\Omega_{c b b}$ can be the $J^{P}=1 / 2^{+}$or $J^{P}=$ $3 / 2^{+}$state. As we will show, various types of weak decays of triply heavy baryons occur, but unfortunately, a universal dynamical (factorization) approach cannot be established yet. This gives a barrier for us to predict their decay widths. Instead, we will use an optional theoretical tool to analyze heavy-quark decays, the flavor $\mathrm{SU}(3)$ symmetry [24-55]. One advantage of the $\mathrm{SU}(3)$ analysis is that it is independent of the factorization details. In this work, we consider semileptonic decay channels with one or two hadrons in the final state, while for nonleptonic decays, the two-body and three-body modes will be analyzed. 
The rest of this paper is organized as follows. Section II is devoted to an estimate of the total decay rates or equivalently the lifetimes that will be used in the calculation of branching fractions. In Sec. III, we will collect the representation matrices for various particle multiplets in the SU(3) group. In Sec. IV, semileptonic decay modes with one or two hadrons in the final state are analyzed. In Secs. V, VI, and VII, nonleptonic decays of $\Omega_{c c c}, \Omega_{b b b}$, $\Omega_{c c b}$, and $\Omega_{c b b}$ will be studied in order. In Sec. VIII, we shall present a collection of golden modes that are most likely to discover the triply heavy baryons. Section IX will briefly discuss the SU(3) symmetry-breaking effects. A short summary is given in the last section.

\section{LIFETIMES AND TOTAL DECAY WIDTHS}

Lifetimes, the inverse of total decay rates, are among the most fundamental properties of the involved triply heavy baryons. The decay rate of a triply heavy baryon $H$ into an inclusive final state $X$ can be expressed as a phase-space integral over the matrix element square of the effective Hamiltonian sandwiched between the initial and final state $X$. Summing over all final states with the same quantum numbers, we obtain

$$
\begin{aligned}
\Gamma(H \rightarrow X)= & \frac{1}{2 m_{H}} \sum_{X} \int \prod_{i}\left[\frac{d^{3} p_{i}}{(2 \pi)^{3} 2 E_{i}}\right] \\
& \times(2 \pi)^{4} \delta^{(4)}\left(p_{H}-p_{X}\right)\left|\left\langle X\left|\mathcal{H}_{\mathrm{eff}}^{e w}\right| H\right\rangle\right|^{2},
\end{aligned}
$$

with $p_{X}=\sum_{i} p_{i}$. The $\mathcal{H}_{\text {eff }}^{e w}$ is the effective electroweak Hamiltonian of which the explicit forms can be found in the following sections. With the help of the optical theorem, the total rate is written as

$$
\Gamma(H \rightarrow X)=\frac{1}{2 m_{H}}\langle H|\mathcal{T}| H\rangle,
$$

with the transition operator

$$
\mathcal{T}=\operatorname{Im} i \int d^{4} x T\left[\mathcal{H}_{\mathrm{eff}}^{e w}(x), \mathcal{H}_{\mathrm{eff}}^{e w}(0)\right]
$$

consisting of a nonlocal double insertion of the effective Hamiltonian.

A theoretical tool that describes the decay widths of inclusive decays is heavy-quark expansion, making use of operator product expansion in essence. Contracting the offshell operators in Eq. (4) results in local operators organized by dimensions. Higher-dimensional operators are suppressed by powers of the inverse of heavy-quark mass $1 / m_{Q}$. Up to dimension 6 , the transition operator is then expanded as

$$
\begin{aligned}
\mathcal{T}= & \frac{G_{F}^{2} m_{Q}^{5}}{192 \pi^{3}}\left|V_{\mathrm{CKM}}\right|^{2}\left[c_{3, Q} \bar{Q} Q+\frac{c_{5, Q}}{m_{Q}^{2}} \bar{Q} g_{s} \sigma_{\mu \nu} G^{\mu \nu} Q\right. \\
& \left.+2 \frac{c_{6, Q}}{m_{Q}^{3}}(\bar{Q} q)_{\Gamma}(\bar{q} Q)_{\Gamma}+\cdots\right],
\end{aligned}
$$

where $G_{F}$ is the Fermi constant and $V_{\mathrm{CKM}}$ is the CabibboKobayashi-Maskawa (CKM) matrix elements. The $c_{i}$ s are the corresponding short-distance coefficients that arise from the semileptonic and nonlepetonic heavy-quark decays. These coefficients have been evaluated to a high accuracy in QCD perturbation theory; please see Ref. [56] for a recent review.

The total decay rate is given by matrix elements of these operators:

$$
\begin{aligned}
\Gamma(H)= & \frac{G_{F} m_{Q}^{5}}{192 \pi^{3}}\left|V_{\mathrm{CKM}}\right|^{2}\left(c_{3, Q} \frac{\langle H|\bar{c} c| H\rangle}{2 m_{H}}\right. \\
& +\frac{c_{5, Q}}{m_{Q}^{2}} \frac{\left\langle H\left|\bar{Q} g_{s} \sigma_{\mu \nu} G^{\mu \nu} Q\right| H\right\rangle}{2 m_{H}} \\
& \left.+\frac{c_{6, Q}}{m_{Q}^{3}} \frac{\left\langle H\left|(\bar{Q} q)_{\Gamma}(\bar{q} Q)_{\Gamma}\right| H\right\rangle}{m_{H}}\right) .
\end{aligned}
$$

\section{A. $\Omega_{c c c}^{++}$}

The dimension- 6 operators arise from the interaction between the decaying heavy quark and the spectator quarks. For the $\Omega_{c c c}^{++}$baryon, such operators do not contribute since two charm quarks cannot scatter at leading order. At the lowest order in $1 / m_{c}$, the $\bar{c} c$ operator gives the charm-quark number in the $\Omega_{c c c}^{++}$baryon:

$$
\frac{\left\langle\Omega_{c c c}^{++}|\bar{c} c| \Omega_{c c c}^{++}\right\rangle}{2 m_{\Omega_{c c c}^{++}}}=3+\mathcal{O}\left(1 / m_{c}\right) .
$$

Other matrix elements suppressed by powers of $1 / m_{c}$ request a detailed model calculation in the future and are omitted in this work. The short-distance coefficient $c_{3, c}$ has been calculated as $c_{3, c}=6.29 \pm 0.72$ at leading order (LO) in QCD and $c_{3, c}=11.61 \pm 1.55$ at next-to-leading order (NLO) [56]. Keeping only the leading-order contribution, we obtain the total decay rate and lifetime:

$$
\begin{aligned}
& \Gamma\left(\Omega_{c c c}^{++}\right)= \begin{cases}(2.18 \pm 0.25) \times 10^{-12} \mathrm{GeV}, & \text { LO } \\
(4.03 \pm 0.54) \times 10^{-12} \mathrm{GeV}, & \text { NLO }\end{cases} \\
& \tau\left(\Omega_{c c c}^{++}\right)= \begin{cases}(302 \pm 35) \times 10^{-15} \mathrm{~s}, & \mathrm{LO} \\
(164 \pm 22) \times 10^{-15} \mathrm{~s}, & \mathrm{NLO}\end{cases}
\end{aligned}
$$

In this equation, we have used the charm-quark mass $m_{c}=1.4 \mathrm{GeV}$. This lifetime, especially at NLO, is smaller than the ones for the charmed mesons: 


$$
\begin{aligned}
\tau\left(D^{ \pm}\right) & =(1040 \pm 7) \times 10^{-15} \mathrm{~s} \\
\tau\left(D^{0}\right) & =(410.1 \pm 1.5) \times 10^{-15} \mathrm{~s} \\
\tau\left(D_{s}^{+}\right) & =(500 \pm 7) \times 10^{-15} \mathrm{~s} .
\end{aligned}
$$

\section{B. $\boldsymbol{\Omega}_{b b b}^{-}$}

The heavy bottom quark is heavier than the charm quark, and thus one would expect the heavy-quark expansion to work better in the $\Omega_{b b b}^{-}$decays. A perturbative calculation of the short-distance coefficient [57]

$$
c_{3, b}= \begin{cases}5.29 \pm 0.35, & \mathrm{LO} \\ 6.88 \pm 0.74, & \mathrm{NLO}\end{cases}
$$

also shows better convergence. Using the above results and $m_{b}=4.8 \mathrm{GeV}$, we obtain the lifetime of the triply bottom baryons:

$$
\begin{gathered}
\Gamma\left(\Omega_{b b b}^{-}\right)=\left\{\begin{array}{ll}
(1.47 \pm 0.01) \times 10^{-12} \mathrm{GeV}, & \text { LO } \\
(1.92 \pm 0.02) \times 10^{-12} \mathrm{GeV}, & \text { NLO }
\end{array},\right. \\
\tau\left(\Omega_{b b b}^{-}\right)= \begin{cases}(0.45 \pm 0.03) \times 10^{-12} \mathrm{~s}, & \text { LO } \\
(0.34 \pm 0.04) \times 10^{-12} \mathrm{~s}, & \text { NLO }\end{cases}
\end{gathered}
$$

As one can see, the lifetime is close to one-third of the ones for the bottomed mesons:

$$
\begin{aligned}
\tau\left(B^{-}\right) & =1.638 \times 10^{-12} \mathrm{~s}, \\
\tau\left(B^{0}\right) & =1.52 \times 10^{-12} \mathrm{~s}, \\
\tau\left(B_{s}^{-}\right) & =1.505 \times 10^{-12} \mathrm{~s} .
\end{aligned}
$$

\section{C. $\Omega_{c c b}^{+}$and $\Omega_{c b b}^{0}$}

The decay rates of $\Omega_{c c b}^{+}$and $\Omega_{c b b}^{0}$ receive both contributions from the charm-quark and bottom-quark decays. Using the formulas and inputs given in the last two subsections, we obtain

$$
\begin{gathered}
\Gamma\left(\Omega_{c c b}^{+}\right)=\left\{\begin{array}{ll}
(1.95 \pm 0.20) \times 10^{-12} \mathrm{GeV}, & \mathrm{LO} \\
(3.32 \pm 0.43) \times 10^{-12} \mathrm{GeV}, & \mathrm{NLO}
\end{array},\right. \\
\tau\left(\Omega_{c c b}^{+}\right)= \begin{cases}(338 \pm 35) \times 10^{-15} \mathrm{~s}, & \mathrm{LO} \\
(198 \pm 25) \times 10^{-15} \mathrm{~s}, & \mathrm{NLO},\end{cases} \\
\Gamma\left(\Omega_{c b b}^{0}\right)= \begin{cases}(1.71 \pm 0.15) \times 10^{-12} \mathrm{GeV}, & \mathrm{LO} \\
(2.62 \pm 0.32) \times 10^{-12} \mathrm{GeV}, & \mathrm{NLO}\end{cases} \\
\tau\left(\Omega_{c b b}^{0}\right)= \begin{cases}(384 \pm 33) \times 10^{-15} \mathrm{~s}, & \mathrm{LO} \\
(251 \pm 30) \times 10^{-15} \mathrm{~s}, & \mathrm{NLO}\end{cases}
\end{gathered}
$$

All these results including the lifetimes for $\Omega_{c c c}^{++}$and $\Omega_{b b b}^{-}$ will be needed in the estimate of branching fractions.

\section{PARTICLE MULTIPLETS}

In this section, we will collect the representations for hadron multiplets under the flavor SU(3) group. We start with the baryon sector. Light baryons made of three light quarks can group into an $\mathrm{SU}(3)$ octet and a decuplet. The light baryon decuplet is symmetric in $\mathrm{SU}(3)$ flavor space and is characterized by the following matrix:

$$
\begin{aligned}
\left(T_{10}\right)^{111}=\Delta^{++}, \quad\left(T_{10}\right)^{112}=\left(T_{10}\right)^{121}=\left(T_{10}\right)^{211}=\frac{1}{\sqrt{3}} \Delta^{+}, & \\
\left(T_{10}\right)^{222} & =\Delta^{-}, \quad\left(T_{10}\right)^{122}=\left(T_{10}\right)^{212}=\left(T_{10}\right)^{221}=\frac{1}{\sqrt{3}} \Delta^{0}, \\
\left(T_{10}\right)^{113} & =\left(T_{10}\right)^{131}=\left(T_{10}\right)^{311}=\frac{1}{\sqrt{3}} \Sigma^{\prime+}, \\
\left(T_{10}\right)^{223} & =\left(T_{10}\right)^{232}=\left(T_{10}\right)^{322}=\frac{1}{\sqrt{3}} \Sigma^{\prime-}, \\
\left(T_{10}\right)^{123} & =\left(T_{10}\right)^{132}=\left(T_{10}\right)^{213}=\left(T_{10}\right)^{231}=\left(T_{10}\right)^{312} \\
& =\left(T_{10}\right)^{321}=\frac{1}{\sqrt{6}} \Sigma^{\prime 0}, \\
\left(T_{10}\right)^{133} & =\left(T_{10}\right)^{313}=\left(T_{10}\right)^{331}=\frac{1}{\sqrt{3}} \Xi^{\prime 0}, \\
\left(T_{10}\right)^{233} & =\left(T_{10}\right)^{323}=\left(T_{10}\right)^{332}=\frac{1}{\sqrt{3}} \Xi^{\prime-}, \\
\left(T_{10}\right)^{333} & =\Omega^{-} .
\end{aligned}
$$

The octet has the expression

$$
T_{8}=\left(\begin{array}{ccc}
\frac{1}{\sqrt{2}} \Sigma^{0}+\frac{1}{\sqrt{6}} \Lambda^{0} & \Sigma^{+} & p \\
\Sigma^{-} & -\frac{1}{\sqrt{2}} \Sigma^{0}+\frac{1}{\sqrt{6}} \Lambda^{0} & n \\
\Xi^{-} & \Xi^{0} & -\sqrt{\frac{2}{3}} \Lambda^{0}
\end{array}\right) .
$$

The triply heavy baryons form an $\mathrm{SU}(3)$ singlet, while doubly heavy baryons are an SU(3) triplet:

$$
\begin{aligned}
T_{c c} & =\left(\begin{array}{c}
\Xi_{c c}^{++}(c c u) \\
\Xi_{c c}^{+}(c c d) \\
\Omega_{c c}^{+}(c c s)
\end{array}\right), \quad T_{b c}=\left(\begin{array}{l}
\Xi_{b c}^{+}(b c u) \\
\Xi_{b c}^{0}(b c d) \\
\Omega_{b c}^{0}(b c s)
\end{array}\right), \\
T_{b b} & =\left(\begin{array}{l}
\Xi_{b b}^{0}(b b u) \\
\Xi_{b b}^{-}(b b d) \\
\Omega_{b b}^{-}(b b s)
\end{array}\right) .
\end{aligned}
$$

Singly charmed and bottom baryons with two light quarks can form an antitriplet or sextet. For the antitriplet and sextet, we have the matrix expression 


$$
\begin{aligned}
T_{\mathbf{c} \overline{\mathbf{3}}}= & \left(\begin{array}{ccc}
0 & \Lambda_{c}^{+} & \Xi_{c}^{+} \\
-\Lambda_{c}^{+} & 0 & \Xi_{c}^{0} \\
-\Xi_{c}^{+} & -\Xi_{c}^{0} & 0
\end{array}\right), \\
T_{\mathbf{c 6}}= & \left(\begin{array}{ccc}
\Sigma_{c}^{++} & \frac{1}{\sqrt{2}} \Sigma_{c}^{+} & \frac{1}{\sqrt{2}} \Xi_{c}^{\prime+} \\
\frac{1}{\sqrt{2}} \Sigma_{c}^{+} & \Sigma_{c}^{0} & \frac{1}{\sqrt{2}} \Xi_{c}^{\prime 0} \\
\frac{1}{\sqrt{2}} \Xi_{c}^{\prime+} & \frac{1}{\sqrt{2}} \Xi_{c}^{\prime 0} & \Omega_{c}^{0}
\end{array}\right) .
\end{aligned}
$$

In the meson sector, a light pseudoscalar meson is formed by a light quark and one light antiquark. It forms an octet,

$$
M_{8}=\left(\begin{array}{ccc}
\frac{\pi^{0}}{\sqrt{2}}+\frac{\eta}{\sqrt{6}} & \pi^{+} & K^{+} \\
\pi^{-} & -\frac{\pi^{0}}{\sqrt{2}}+\frac{\eta}{\sqrt{6}} & K^{0} \\
K^{-} & \bar{K}^{0} & -\frac{2 \eta}{\sqrt{6}}
\end{array}\right),
$$

while the singlet $\eta_{1}$ is not considered. Our analysis is also applicable to light vector mesons and other light mesons. The charmed meson forms an SU(3) antitriplet,

$$
D_{i}=\left(\begin{array}{lll}
D^{0}, & D^{+}, & D_{s}^{+}
\end{array}\right)
$$

and the anticharmed meson forms an SU(3) triplet,

$$
\bar{D}^{i}=\left(\bar{D}^{0}, \quad D^{-}, \quad D_{s}^{-}\right)
$$

The above two SU(3) triplets are also applicable to the bottom mesons.

In the following, we will construct the hadron-level effective Hamiltonian for various decay modes. It is necessary to point out that a hadron in the final state must be created by its antiparticle field. For instance, to produce a $\Xi_{c c u}^{++}$, one must use the $\overline{\Xi_{c c u}^{++}}$in the Hamiltonian, and the doubly heavy baryon antitriplet is abbreviated as $\bar{T}_{c c}$.

\section{SEMILEPTONIC DECAY CHANNELS}

\section{A. Semileptonic $\boldsymbol{\Omega}_{c c c}$ decays}

The $c \rightarrow q \bar{\ell} \nu$ transition is induced by the effective electroweak Hamiltonian

$\mathcal{H}_{\text {e.w. }}=\frac{G_{F}}{\sqrt{2}}\left[V_{c q}^{*} \bar{q} \gamma^{\mu}\left(1-\gamma_{5}\right) c \bar{\nu}_{\ell} \gamma_{\mu}\left(1-\gamma_{5}\right) \ell\right]+$ H.c.,

where $q=d, s$ and $\ell=e, \mu$. The $V_{c d}$ and $V_{c s}$ are CKM matrix elements. Heavy-to-light operators form an SU(3) triplet, denoted as $\mathrm{H}_{3}$ with the nonzero components $\left(H_{3}\right)^{2}=V_{c d}^{*},\left(H_{3}\right)^{3}=V_{c s}^{*}$.

At the hadron level, the effective Hamiltonian for threebody and four-body semileptonic $\Omega_{c c c}$ decays can be constructed as

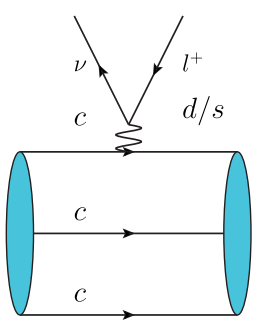

(a)

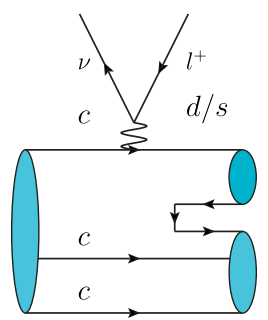

(b)

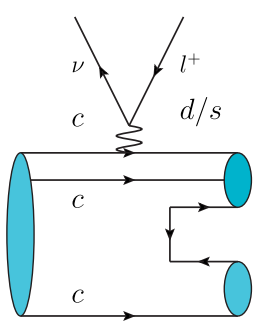

(c)
FIG. 1. Feynman diagrams for semileptonic decays of $\Omega_{c c c}^{++}$. Panel (a) corresponds to three-body decay modes with a doubly charmed baryon in the final state. In panel (b), one additional light meson is generated. The last panel corresponds to the decays into a charmed baryon and charmed meson.

$$
\begin{aligned}
\mathcal{H}_{\mathrm{eff}}= & a_{1} \Omega_{c c c}\left(\bar{T}_{c c}\right)_{i}\left(H_{3}\right)^{i} \bar{\nu}_{\ell} \ell \\
& +a_{2} \Omega_{c c c}\left(\bar{T}_{c c}\right)_{i}\left(M_{8}\right)_{j}^{i}\left(H_{3}\right)^{j} \bar{\nu}_{\ell} \ell \\
& +a_{3} \Omega_{c c c}\left(\bar{T}_{c \overline{3}}\right)_{[i j]} \bar{D}^{i}\left(H_{3}\right)^{j} \bar{\nu}_{\ell} \ell \\
& +a_{4} \Omega_{c c c}\left(\bar{T}_{c 6}\right)_{\{i j\}} \bar{D}^{i}\left(H_{3}\right)^{j} \bar{\nu}_{\ell} \ell,
\end{aligned}
$$

where the $a_{i}$ 's are SU(3) irreducible amplitudes. Feynman diagrams for these modes are given in Fig. 1. Decay amplitudes for various channels can be deduced from the Hamiltonian in Eq. (25), and are collected in Table I.

A few remarks are given in order:

(i) In this table and the following ones, the light pseudoscalar mesons can be replaced by their vector counterparts. For instance, the $K^{0}$ can be replaced by a $K^{* 0}$, which is reconstructed by the $K^{-} \pi^{+}$final state. The $\eta$ meson is difficult to reconstruct at hadron colliders, while the vector $\phi$ meson can be reconstructed in the $K^{+} K^{-}$final state with high efficiency.

(ii) The $c \rightarrow s$ transition is proportional to the $V_{c s} \sim 1$, and the $c \rightarrow d$ transition has a smaller CKM matrix

TABLE I. Amplitudes for semileptonic $\Omega_{c c c}$ decays derived from Eq. (25). The light meson in the final state can be replaced by its vector counterpart. For instance, the $K^{0}$ can be replaced by a $K^{* 0}$ decaying into $K^{-} \pi^{+}$.

\begin{tabular}{lccc}
\hline \hline Channel & Amplitude & Channel & Amplitude \\
\hline$\Omega_{c c c}^{++} \rightarrow \Xi_{c c}^{+} \ell^{+} \nu_{\ell}$ & $a_{1} V_{c d}^{*}$ & $\Omega_{c c c}^{++} \rightarrow \Lambda_{c}^{+} D^{0} \ell^{+} \nu_{\ell}$ & $a_{3} V_{c d}^{*}$ \\
$\Omega_{c c c}^{++} \rightarrow \Omega_{c c}^{+} \ell^{+} \nu_{\ell}$ & $a_{1} V_{c s}^{*}$ & $\Omega_{c c c}^{++} \rightarrow \Xi_{c}^{+} D^{0} \ell^{+} \nu_{\ell}$ & $a_{3} V_{c s}^{*}$ \\
$\Omega_{c c c}^{++} \rightarrow \Xi_{c c}^{++} \pi^{-} \ell^{+} \nu_{\ell}$ & $a_{2} V_{c d}^{*}$ & $\Omega_{c c c}^{++} \rightarrow \Xi_{c}^{0} D^{+} \ell^{+} \nu_{\ell}$ & $a_{3} V_{c s}^{*}$ \\
$\Omega_{c c c}^{++} \rightarrow \Xi_{c c}^{++} K^{-} \ell^{+} \nu_{\ell}$ & $a_{2} V_{c s}^{*}$ & $\Omega_{c c}^{++} \rightarrow \Xi_{c}^{0} D_{s}^{+} \ell^{+} \nu_{\ell}$ & $-a_{3} V_{c d}^{*}$ \\
$\Omega_{c c c}^{++} \rightarrow \Xi_{c c}^{+} \pi^{0} \ell^{+} \nu_{\ell}$ & $-\frac{a_{2} V_{c d}^{*}}{\sqrt{2}}$ & $\Omega_{c c c}^{++} \rightarrow \Sigma_{c}^{+} D^{0} \ell^{+} \nu_{\ell}$ & $\frac{a_{4} V_{c d}^{*}}{\sqrt{2}}$ \\
$\Omega_{c c c}^{++} \rightarrow \Xi_{c c}^{+} \bar{K}^{0} \ell^{+} \nu_{\ell}$ & $a_{2} V_{c s}^{*}$ & $\Omega_{c c}^{++} \rightarrow \Sigma_{c}^{0} D^{+} \ell^{+} \nu_{\ell}$ & $a_{4} V_{c d}^{*}$ \\
$\Omega_{c c c}^{++} \rightarrow \Xi_{c c}^{+} \eta \ell^{+} \nu_{\ell}$ & $\frac{a_{2} V_{c d}^{*}}{\sqrt{6}}$ & $\Omega_{c c c}^{++} \rightarrow \Xi_{c}^{\prime+} D^{0} \ell^{+} \nu_{\ell}$ & $\frac{a_{4} V_{c s}^{*}}{\sqrt{2}}$ \\
$\Omega_{c c c}^{++} \rightarrow \Omega_{c c}^{+} K^{0} \ell^{+} \nu_{\ell}$ & $a_{2} V_{c d}^{*}$ & $\Omega_{c c c}^{++} \rightarrow \Xi_{c}^{\prime 0} D^{+} \ell^{+} \nu_{\ell}$ & $\frac{a_{4} V_{c s}^{*}}{\sqrt{2}}$ \\
$\Omega_{c c c}^{++} \rightarrow \Omega_{c c}^{+} \eta \ell^{+} \nu_{\ell}$ & $-\sqrt{\frac{2}{3} a_{2} V_{c s}^{*}}$ & $\Omega_{c c c}^{++} \rightarrow \Xi_{c}^{\prime 0} D_{s}^{+} \ell^{+} \nu_{\ell}$ & $\frac{a_{4} V_{c d}^{*}}{\sqrt{2}}$ \\
& $\Omega_{c c c}^{++} \rightarrow \Omega_{c}^{0} D_{s}^{+} \ell^{+} \nu_{\ell}$ & $a_{4} V_{c s}^{*}$ \\
\hline \hline
\end{tabular}


element $V_{c d} \sim 0.2$. Inspired by the experimental data on $D$-meson decays [6], we can infer that branching fractions for the $c \rightarrow s$ channels are about a few percent, and the ones for the $c \rightarrow d$ transitions are at the order $10^{-3}$.

(iii) A number of relations for decay widths can be easily read off from Table I. For instance, for the $c \rightarrow s$ decays into a doubly charmed baryon, we have

$$
\begin{aligned}
& \Gamma\left(\Omega_{c c c}^{++} \rightarrow \Xi_{c c}^{++} K^{-} \ell^{+} \nu_{\ell}\right) \\
& \quad=\Gamma\left(\Omega_{c c c}^{++} \rightarrow \Xi_{c c}^{+} \bar{K}^{0} \ell^{+} \nu_{\ell}\right) \\
& \quad=\frac{3}{2} \Gamma\left(\Omega_{c c c}^{++} \rightarrow \Omega_{c c}^{+} \eta \ell^{+} \nu_{\ell}\right) .
\end{aligned}
$$

However, it is necessary to point out that the above relations will be modified due to the different masses of the final hadrons. Once the mass of $\Omega_{c c c}$ is experimentally measured in the future, kinematical corrections can be included, and these relations can be refined.

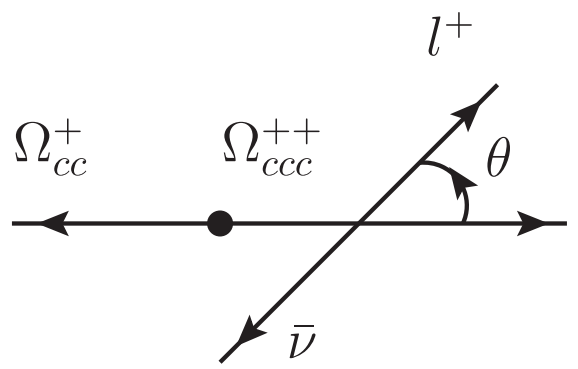

FIG. 2. Kinematics for the $\Omega_{c c c}^{++} \rightarrow \Omega_{c c}^{+} l^{+} \bar{\nu}$ decay. In the $\Omega_{c c c}^{++}$ rest frame, the $\Omega_{c c}^{+}$moves on the opposite of the $z$ axis. In the lepton pair $l^{+} \bar{\nu}$ rest frame, the $l^{+}$flying direction and the $z$ axis intersect with the polar angle $\theta$.

There is only one final hadron in the $\Omega_{c c c}^{++} \rightarrow$ $\Xi_{c c}^{+} / \Omega_{c c}^{+} \ell^{+} \nu_{\ell}$ decays. Leptonic amplitudes can be calculated in electroweak perturbation theory, while the hadronic matrix element can be parametrized in terms of form factors,

$$
\begin{aligned}
\left\langle\Omega_{c c}^{+}\left(p^{\prime}\right)\left|\bar{s} \gamma^{\mu} \gamma_{5} c\right| \Omega_{c c c}^{++}(p)\right\rangle & =\bar{u}\left(p^{\prime}\right)\left[\frac{f_{1} \gamma^{\mu} P^{\alpha}}{m_{\Omega_{c c c}^{++}}-m_{\Omega_{c c}^{+}}}+\frac{f_{2} P^{\mu} P^{\alpha}}{m_{\Omega_{c c c}^{++}}^{2+}-m_{\Omega_{c c}^{+}}^{2}}+\frac{i f_{3} \sigma^{\mu \nu} q_{\nu} P^{\alpha}}{m_{\Omega_{c c c}^{++}}^{2+}}+f_{4} g^{\alpha \mu}\right] u^{\alpha}(p), \\
\left\langle\Omega_{c c}^{+}\left(p^{\prime}\right)\left|\bar{s} \gamma^{\mu} c\right| \Omega_{c c c}^{++}(p)\right\rangle & =\bar{u}\left(p^{\prime}\right)\left[\frac{g_{1} \gamma^{\mu} P^{\alpha}}{m_{\Omega_{c c c}^{++}}+m_{\Omega_{c c}^{+}}}+\frac{g_{2} P^{\mu} P^{\alpha}}{m_{\Omega_{c c c}^{++}}^{2}-m_{\Omega_{c c}^{+}}^{2}}+\frac{i g_{3} \sigma^{\mu \nu} q_{\nu} P^{\alpha}}{m_{\Omega_{c c c}^{+}}^{2}}+g_{4} g^{\alpha \mu}\right] \gamma_{5} u^{\alpha}(p),
\end{aligned}
$$

with $P=p+p^{\prime}$ and $q=p-p^{\prime}$. Here, $u(p)$ is the spinor for the final $1 / 2^{+}$baryon, and $u_{\alpha}\left(p^{\prime}\right)$ is the Rartia-Schwinger vectorial spinor for the initial $3 / 2^{+}$baryon, satisfying

$$
\not p u^{\alpha}(p)=m_{\Omega_{c c}^{++}} u^{\alpha}(p), \quad p_{\alpha} u^{\alpha}(p)=\gamma_{\alpha} u^{\alpha}(p)=0 .
$$

It can be constructed using the polarization vector and a spinor,

$$
u^{\alpha}(p)=\left(\epsilon^{\alpha}-\frac{1}{3}\left(\gamma^{\alpha}+\frac{p^{\alpha}}{m}\right) \phi\right) u(p)
$$

which is a product of the Clebsch-Gordon coefficient. The partial decay widths for $\Omega_{c c c}^{++} \rightarrow \Omega_{c c}^{+} / \Xi_{c c}^{+} l^{+} \bar{\nu}$ are then derived as

$$
\begin{aligned}
\frac{d \Gamma}{d q^{2} d \cos \theta}= & \frac{\sqrt{\lambda} G_{F}^{2} q^{2}}{1024 \pi^{3} m_{I}^{3}}\left(1-\frac{m_{l}^{2}}{q^{2}}\right)^{2}\left(\left(m_{I}+m_{f}\right)^{2}-q^{2}\right)\left|V_{\mathrm{CKM}}\right|^{2}\left\{\left(\left|H_{1,-1 / 2}^{V, 3 / 2}\right|^{2}+\left|H_{1,-1 / 2}^{A, 3 / 2}\right|^{2}+\left|H_{1,1 / 2}^{V, 1 / 2}\right|^{2}+\left|H_{1,1 / 2}^{A, 1 / 2}\right|^{2}\right)\right. \\
& \times\left(1+\cos ^{2} \theta+\frac{m_{l}^{2}}{q^{2}} \sin ^{2} \theta\right)-4 \cos \theta \operatorname{Re}\left[H_{1,-1 / 2}^{V, 3 / 2}\left(H_{1,-1 / 2}^{A, 3 / 2}\right)^{*}+H_{1,1 / 2}^{V, 1 / 2}\left(H_{1,1 / 2}^{A, 1 / 2}\right)^{*}\right]+\left(\left|H_{0,-1 / 2}^{V, 1 / 2}\right|^{2}+\left|H_{0,-1 / 2}^{A, 1 / 2}\right|^{2}\right) \\
& \times\left(2 \sin ^{2} \theta+\frac{2 m_{l}^{2}}{q^{2}} \cos ^{2} \theta\right)+\left(\left|H_{t,-1 / 2}^{V, 1 / 2}\right|^{2}+\left|H_{t,-1 / 2}^{A, 1 / 2}\right|^{2}\right) \frac{2 m_{l}^{2}}{q^{2}}-4 \cos \theta \frac{m_{l}^{2}}{q^{2}}\left(\operatorname { R e } \left[H_{0,-1 / 2}^{V, 1 / 2}\left(H_{t,-1 / 2}^{V, 1 / 2}\right)^{*}\right.\right. \\
& \left.\left.\left.+H_{0,-1 / 2}^{A, 1 / 2}\left(H_{t,-1 / 2}^{A, 1 / 2}\right)^{*}\right]\right)\right\}
\end{aligned}
$$

where the kinematics is chosen as in Fig. 2. $m_{I}=m_{\Omega_{c c}^{+}}$, and $m_{f}=m_{\Omega_{c c}^{+}}$or $m_{\Xi_{c c}^{+}}$. In the above, the $H_{i}$ 's are the hadronic helicity amplitudes: 


$$
\begin{gathered}
H_{1,-1 / 2}^{V, 3 / 2}=H_{-1,1 / 2}^{V,-3 / 2}=f_{4}, \quad H_{1,-1 / 2}^{A, 3 / 2}=-H_{-1,-1 / 2}^{A,-3 / 2}=\frac{g_{4} \sqrt{\lambda}}{\hat{f}_{+}}, \\
H_{0,-1 / 2}^{V, 1 / 2}=H_{0,1 / 2}^{V,-1 / 2}=-\frac{f_{1}\left(1+m_{f}\right) \hat{f}_{-}}{\sqrt{6}\left(1-m_{f}\right) \sqrt{q^{2}}}-\frac{\lambda f_{2}}{\sqrt{6}\left(1-m_{f}^{2}\right) \sqrt{q^{2}}}+\frac{f_{3} \hat{f}_{-} \sqrt{q^{2}}}{\sqrt{6}}+\frac{f_{4}\left(1+q^{2}-m_{f}^{2}\right)}{\sqrt{6} \sqrt{q^{2}}}, \\
H_{0,-1 / 2}^{A, 1 / 2}=-H_{0,1 / 2}^{A,-1 / 2}=\frac{g_{1} \lambda\left(1-m_{f}\right)}{\sqrt{6}\left(1+m_{f}\right) \sqrt{q^{2}}}-\frac{g_{2} \sqrt{\lambda} \hat{f}_{-}}{\sqrt{6}\left(1-m_{f}^{2}\right) \sqrt{q^{2}}}+\frac{g_{3} \sqrt{\lambda} \sqrt{q^{2}}}{\sqrt{6}}+\frac{g_{4} \sqrt{\lambda}\left(1+q^{2}-m_{f}^{2}\right)}{\sqrt{6} \hat{f}_{+} \sqrt{q^{2}}}, \\
H_{t,-1 / 2}^{V, 1 / 2}=H_{t, 1 / 2}^{V,-1 / 2}=-\frac{\left(f_{1}+f_{2}-f_{4}\right) \sqrt{\lambda}}{\sqrt{6 q^{2}}}, \\
H_{t,-1 / 2}^{A, 1 / 2}=-H_{t, 1 / 2}^{A,-1 / 2}=\frac{\left(g_{1}-g_{2}+g_{4}\right) \hat{f}_{-}}{\sqrt{6 q^{2}}}, \\
H_{1,1 / 2}^{V, 1 / 2}=H_{-1,-1 / 2}^{V,-1 / 2}=-\frac{f_{1} \hat{f}_{-}}{\sqrt{3}\left(1-m_{f}\right)}+\frac{f_{3}\left(1+m_{f}\right) \hat{f}_{-}}{\sqrt{3}}+\frac{f_{4}}{\sqrt{3}} \\
H_{1,1 / 2}^{A, 1 / 2}=-H_{-1,-1 / 2}^{A,-1 / 2}=-\frac{g_{1} \sqrt{\lambda}}{\sqrt{3}\left(1+m_{f}\right)}-\frac{g_{3} \sqrt{\lambda}\left(1-m_{f}\right)}{\sqrt{3}}-\frac{f_{4} \sqrt{\lambda}}{\sqrt{3} \hat{f}_{+}}
\end{gathered}
$$

A factor $i \sqrt{2 m_{I}\left(E_{f}+m_{f}\right)}=i \sqrt{\left(m_{I}+m_{f}\right)^{2}-q^{2}}$ has been taken out in the amplitudes. $m_{f}=m_{f} / m_{I}$, and $q^{2}=q^{2} / m_{I}^{2}$. The $\lambda$ function is introduced as

$\lambda \equiv \lambda\left(m_{I}^{2}, m_{f}^{2}, q^{2}\right)=\left(m_{I}^{2}-m_{f}^{2}-q^{2}\right)^{2}-4 m_{f}^{2} q^{2}$,

$\lambda \equiv \lambda\left(1, m_{f}^{2}, q^{2}\right)=\left(1-m_{f}^{2}-q^{2}\right)^{2}-4 m_{f}^{2} q^{2}$,

and the abbreviation has been introduced:

$\hat{f}_{+}=\left(1+m_{f}\right)^{2}-q^{2}, \quad \hat{f}_{-}=\left(1-m_{f}\right)^{2}-q^{2}$.

In the above transitions, the two heavy quarks act as spectators and might be viewed as a diquark system. The quark-diquark correspondence implies that the above transition might be related to the $B_{c} \rightarrow B$ transition, in which the bottom quark acts a spectator. Using the $B_{c} \rightarrow B$ form factors as a very rough approximation [58], we obtain an order-of-magnitude estimate of partial decay widths:

$$
\begin{aligned}
& \Gamma\left(\Omega_{c c c}^{++} \rightarrow \Omega_{c c}^{+} l^{+} \nu\right)=1.41 \times 10^{-13} \mathrm{GeV}, \\
& \Gamma\left(\Omega_{c c c}^{++} \rightarrow \Xi_{c c}^{+} l^{+} \nu\right)=1.29 \times 10^{-14} \mathrm{GeV} .
\end{aligned}
$$

Masses for triply and doubly heavy baryons are taken from the QCD sum rules calculations [13,59]:

$$
\begin{aligned}
m_{\Omega_{c c c}^{++}} & =4.99 \mathrm{GeV}, \quad m_{\Xi_{c c}^{+}}=3.57 \mathrm{GeV}, \\
m_{\Omega_{c c}^{+}} & =3.71 \mathrm{GeV} .
\end{aligned}
$$

Notice the mass for $\Xi_{c c}^{+}$is slightly below the experimental result for $m_{\Xi_{c c}^{++}}$shown in Eq. (1), but this difference will not cause sizable modifications to decay widths. Using the NLO lifetime given in Eq. (13), we can derive the branching fractions:

$$
\begin{aligned}
\mathcal{B}\left(\Omega_{c c c}^{++} \rightarrow \Omega_{c c}^{+} l^{+} \nu\right) & =3.6 \%, \\
\mathcal{B}\left(\Omega_{c c c}^{++} \rightarrow \Xi_{c c}^{+} l^{+} \nu\right) & =0.33 \% .
\end{aligned}
$$

It is worth noting that the CKM matrix element of $\Omega_{c c c}^{++} \rightarrow \Omega_{c c}^{+} l^{+} \nu$ is $\left|V_{\text {cs }}\right|^{2}$ and matrix element of $\Omega_{c c c}^{++} \rightarrow$ $\Xi_{c c}^{+} l^{+} \nu$ is $\left|V_{\text {cd }}\right|^{2}$. Numerically, $\left|V_{\text {cs }}\right|^{2} /\left|V_{\text {cd }}\right|^{2} \sim 20$, but due to the larger phase-space region of the latter process, the ratio of these two branching fractions is about 10 , as one can see in the equations above.

\section{B. Semileptonic $\boldsymbol{\Omega}_{b b b}$ decays}

The $b$-quark decay is controlled by the electroweak Hamiltonian

$\mathcal{H}_{\text {e.e. }}=\frac{G_{F}}{\sqrt{2}}\left[V_{q^{\prime} b} \bar{q}^{\prime} \gamma^{\mu}\left(1-\gamma_{5}\right) b \bar{\ell} \gamma_{\mu}\left(1-\gamma_{5}\right) \nu_{\ell}\right]+$ H.c.,

with $q^{\prime}=u, c$, and here $\ell=e, \mu, \tau$. The $b \rightarrow c$ transition is an $\mathrm{SU}(3)$ singlet, and thus the transition is simply a singlet. The $b \rightarrow u$ transition forms an $\mathrm{SU}(3)$ triplet $H_{3}^{\prime}$ with $\left(H_{3}^{\prime}\right)^{1}=1$ and $\left(H_{3}^{\prime}\right)^{2,3}=0$. The hadron-level Hamiltonian is given as 


$$
\begin{aligned}
\mathcal{H}_{\text {eff }}= & b_{1} \Omega_{b b b} \bar{\Omega}_{c b b} \bar{\ell} \nu_{\ell}+b_{2} \Omega_{b b b}\left(\bar{T}_{b b}\right)_{i} \bar{D}^{i} \bar{\ell} \nu_{\ell} \\
& +b_{3} \Omega_{b b b}\left(\bar{T}_{b c}\right)_{i} \bar{B}^{i} \bar{\ell} \nu_{\ell}+b_{4} \Omega_{b b b}\left(\bar{T}_{b b}\right)_{i}\left(H_{3}^{\prime}\right)^{i} \bar{\ell} \nu_{\ell} \\
& +b_{5} \Omega_{b b b}\left(\bar{T}_{b b}\right)_{i}\left(M_{8}\right)_{j}^{i}\left(H_{3}^{\prime}\right)^{j} \bar{\ell} \nu_{l} \\
& +b_{6} \Omega_{b b b}\left(\bar{T}_{b \overline{3}}\right)_{[i j]} \bar{B}^{i}\left(H_{3}^{\prime}\right)^{j} \bar{\ell} \nu_{\ell} \\
& +b_{7} \Omega_{b b b}\left(\bar{T}_{b 6}\right)_{\{i j\}} \bar{B}^{i}\left(H_{3}^{\prime}\right)^{j} \bar{\ell} \nu_{\ell} .
\end{aligned}
$$

The $b_{i}$ 's are the $\mathrm{SU}(3)$-independent amplitudes like the $a_{i}$ 's in Eq. (25). Feynman diagrams are shown in Fig. 3. Decay amplitudes can be deduced from this Hamiltonian, and the results are given in Table II.

A few remarks are given in order:

(i) The $\Omega_{c b b}$ in the final state can be $1 / 2^{+}$or $3 / 2^{+}$. It is similar for $T_{b b}, T_{b c}$, and others.

(ii) Decay modes induced by the $b \rightarrow c$ transition still contain a triply heavy baryon, which must be detected through its subsequent weak decays.

\section{Semileptonic $\boldsymbol{\Omega}_{c c b}$ decays}

Both the charm quark and bottom quark in $\Omega_{c c b}$ can weakly decay. Thus, the hadron-level Hamiltonian for semileptonic $\Omega_{c c b}$ decays is given as

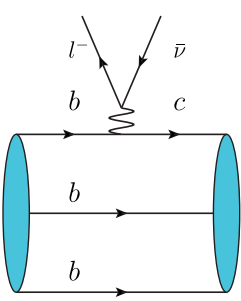

(a)

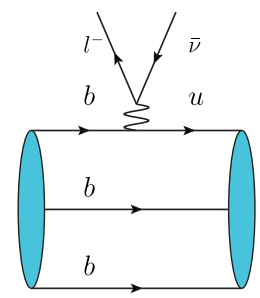

(d)

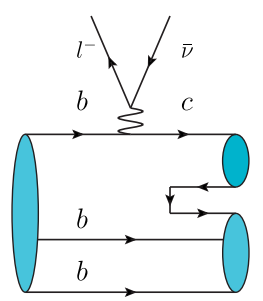

(b)

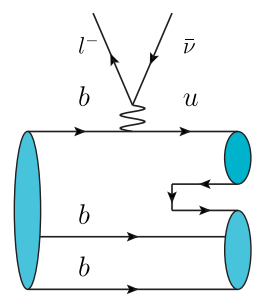

(e)

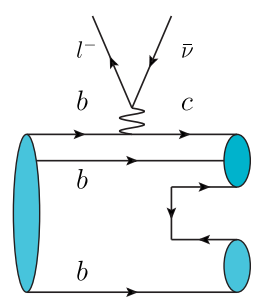

(c)

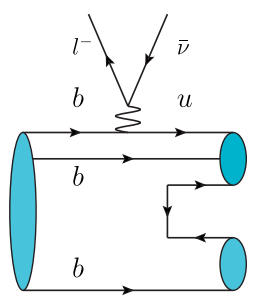

(f)
FIG. 3. Feynman diagrams for semileptonic decays of $\Omega_{b b b}^{-}$. Panel (a) corresponds to three-body decay modes with a doubly charmed baryon in the final state. In panel (b), one additional light meson is generated. The last panel corresponds to the decays into a charmed baryon and charmed meson.

$$
\begin{aligned}
\mathcal{H}_{\mathrm{eff}}= & c_{1} \Omega_{c c b}\left(\bar{T}_{b c}\right)_{i}\left(H_{3}\right)^{i} \bar{\nu}_{\ell} \ell+c_{2} \Omega_{c c b}\left(\bar{T}_{b c}\right)_{i}\left(M_{8}\right)_{j}^{i}\left(H_{3}\right)^{j} \bar{\nu}_{\ell} \ell+c_{3} \Omega_{c c b}\left(\bar{T}_{b \overline{3}}\right)_{[i j]} \bar{D}^{i}\left(H_{3}\right)^{j} \bar{\nu}_{\ell} \ell+c_{4} \Omega_{c c b}\left(\bar{T}_{c \overline{3}}\right)_{[i j]} \bar{B}^{i}\left(H_{3}\right)^{j} \bar{\nu}_{\ell} \ell \\
& +c_{5} \Omega_{c c b}\left(\bar{T}_{b 6}\right)_{\{i j\}} \bar{D}^{i}\left(H_{3}\right)^{j} \bar{\nu}_{\ell} \ell+c_{6} \Omega_{c c b}\left(\bar{T}_{c 6}\right)_{\{i j\}} \bar{B}^{i}\left(H_{3}\right)^{j} \bar{\nu}_{\ell} \ell+c_{7} \Omega_{c c b} \bar{\Omega}_{c c c} \bar{\ell} \nu_{\ell}+c_{8} \Omega_{c c b}\left(\bar{T}_{c c}\right)_{i} \bar{D}^{i} \bar{\ell} \nu_{\ell} \\
& +c_{9} \Omega_{c c b}\left(\bar{T}_{c c}\right)_{i}\left(H_{3}^{\prime}\right)^{i} \bar{\ell} \nu_{\ell}+c_{10} \Omega_{c c b}\left(\bar{T}_{c c}\right)_{i}\left(M_{8}\right)_{j}^{i}\left(H_{3}^{\prime}\right)^{j} \bar{\ell} \nu_{l}+c_{11} \Omega_{c c b}\left(\bar{T}_{c}\right)_{[i j]} \bar{D}^{i}\left(H_{3}^{\prime}\right)^{j} \bar{\ell} \nu_{\ell} \\
& +c_{12} \Omega_{c c b}\left(\bar{T}_{c 6}\right)_{\{i j\}} \bar{D}^{i}\left(H_{3}^{\prime}\right)^{j} \bar{\ell} \nu_{\ell} .
\end{aligned}
$$

Decay amplitudes for different channels are obtained by expanding the above Hamiltonian and are collected in Table III.

\section{Semileptonic $\boldsymbol{\Omega}_{c b b}$ decays}

Similarly, the hadron-level Hamiltonian for semileptonic $\Omega_{c b b}$ decay is given as

$$
\begin{aligned}
\mathcal{H}_{\mathrm{eff}}= & d_{1} \Omega_{c b b} \Omega_{c c b} \bar{\ell} \nu_{\ell}+d_{2} \Omega_{c b b}\left(\bar{T}_{c c}\right)_{i} \bar{B}^{i} \bar{\ell} \nu_{\ell}+d_{3} \Omega_{c b b}\left(\bar{T}_{b c}\right)_{i} \bar{D}^{i} \bar{\ell} \nu_{\ell}+d_{4} \Omega_{c b b}\left(T_{b c}\right)_{i}\left(H_{3}^{\prime}\right)^{i} \bar{\ell} \nu_{\ell}+d_{5} \Omega_{c b b}\left(T_{b c}\right)_{i} M_{j}^{i}\left(H_{3}^{\prime}\right)^{j} \bar{\ell} \nu_{l} \\
& +d_{6} \Omega_{c b b}\left(\bar{T}_{b \overline{3}}\right)_{[i j]} \bar{D}^{i}\left(H_{3}^{\prime}\right)^{j} \bar{\ell} \nu_{\ell}+d_{7} \Omega_{c b b}\left(\bar{T}_{c \overline{3}}\right)_{[i j]} \bar{B}^{i}\left(H_{3}^{\prime}\right)^{j} \bar{\ell} \nu_{\ell}+d_{8} \Omega_{c b b}\left(\bar{T}_{b 6}\right)_{\{i j\}} \bar{D}^{i}\left(H_{3}^{\prime}\right)^{j} \bar{\ell} \nu_{\ell} \\
& +d_{9} \Omega_{c b b}\left(\bar{T}_{c 6}\right)_{\{i j\}} \bar{B}^{i}\left(H_{3}^{\prime}\right)^{j} \bar{\ell} \nu_{\ell}+d_{10} \Omega_{c b b}\left(T_{b b}\right)_{i}\left(H_{3}\right)^{i} \bar{\nu}_{\ell} \ell+d_{11} \Omega_{c b b}\left(T_{b b}\right)_{i} M_{j}^{i}\left(H_{3}\right)^{j} \bar{\nu}_{\ell} \ell \\
& +d_{12} \Omega_{c b b}\left(\bar{T}_{b \overline{3}}\right)_{[i j]} \bar{B}^{i}\left(H_{3}\right)^{j} \bar{\nu}_{\ell} \ell+d_{13} \Omega_{c b b}\left(\bar{T}_{b 6}\right)_{\{i j\}} \bar{B}^{i}\left(H_{3}\right)^{j} \bar{\nu}_{\ell} \ell .
\end{aligned}
$$

TABLE II. Decay amplitudes for $\Omega_{b b b}$ semileptonic decays with one or two hadrons in the final state.

\begin{tabular}{lccccc}
\hline \hline Channel & Amplitude & Channel & Amplitude & Channel & Amplitude \\
\hline$\Omega_{b b b}^{-} \rightarrow \Omega_{c b b}^{0} \ell^{-} \bar{\nu}_{\ell}$ & $b_{1} V_{c b}$ & $\Omega_{b b b}^{-} \rightarrow \Xi_{b b}^{0} \ell^{-} \bar{\nu}_{\ell}$ & $b_{4} V_{u b}$ & & \\
$\Omega_{b b b}^{-} \rightarrow \Xi_{b b}^{0} D^{0} \ell^{-} \bar{\nu}_{\ell}$ & $b_{2} V_{c b}$ & $\Omega_{b b b}^{-} \rightarrow \Xi_{b b}^{-} D^{+} \ell^{-} \bar{\nu}_{\ell}$ & $b_{2} V_{c b}$ & $\Omega_{b b b}^{-} \rightarrow \Omega_{b b}^{-} D_{s}^{+} \ell^{-} \bar{\nu}_{\ell}$ & $b_{2} V_{c b}$ \\
$\Omega_{b b b}^{-} \rightarrow \Xi_{b b}^{0} \bar{B}^{0} \ell^{-} \bar{\nu}_{\ell}$ & $b_{3} V_{c b}$ & $\Omega_{b b b}^{-} \rightarrow \Omega_{b c}^{0} \bar{B}_{s}^{0} \ell^{-} \bar{\nu}_{\ell}$ & $b_{3} V_{c b}$ & $\Omega_{b b b}^{-} \rightarrow \Xi_{b c}^{+} B^{-} \ell^{-} \bar{\nu}_{\ell}$ & $b_{3} V_{c b}$ \\
$\Omega_{b b b}^{-} \rightarrow \Xi_{b b}^{0} \pi^{0} \ell^{-} \bar{\nu}_{\ell}$ & $b_{5} V_{u b}$ & $\Omega_{b b b}^{-} \rightarrow \Xi_{b b}^{0} \eta \ell^{-} \bar{\nu}_{\ell}$ & $\frac{b_{5} V_{u b}}{\sqrt{6}}$ & $\Omega_{b b b}^{-} \rightarrow \Xi_{b b}^{-} \pi^{+} \ell^{-} \bar{\nu}_{\ell}$ & $b_{5} V_{u b}$ \\
$\Omega_{b b b}^{-} \rightarrow \Omega_{b b}^{-} K^{+} \ell^{-} \bar{\nu}_{\ell}$ & $b_{5} V_{u b}$ & $\Omega_{b b b}^{-} \rightarrow \Lambda_{b}^{0} \bar{B}^{0} \ell^{-} \bar{\nu}_{\ell}$ & $-b_{6} V_{u b}$ & $\Omega_{b b b}^{-} \rightarrow \Xi_{b}^{0} \bar{B}_{s}^{0} \ell^{-} \bar{\nu}_{\ell}$ & $-b_{6} V_{u b}$ \\
$\Omega_{b b b}^{-} \rightarrow \Sigma_{b}^{+} B^{-} \ell^{-} \bar{\nu}_{\ell}$ & $b_{7} V_{u b}$ & $\Omega_{b b b}^{-} \rightarrow \Sigma_{b}^{0} \bar{B}^{0} \ell^{-} \bar{\nu}_{\ell}$ & $\frac{b_{7} V_{u b}}{\sqrt{2}}$ & $\Omega_{b b b}^{-} \rightarrow \Xi_{b}^{\prime 0} \bar{B}_{s}^{0} \ell^{-} \bar{\nu}_{\ell}$ & $\frac{b_{7} V_{u b}}{\sqrt{2}}$ \\
\hline \hline
\end{tabular}


TABLE III. Decay amplitudes for $\Omega_{c c b}^{+}$semileptonic decays.

\begin{tabular}{|c|c|c|c|}
\hline Channel & Amplitude & Channel & Amplitude \\
\hline$\Omega_{c c b}^{+} \rightarrow \Omega_{c c c}^{++} \ell^{-} \bar{\nu}_{\ell}$ & $c_{7} V_{c b}$ & $\Omega_{c c b}^{+} \rightarrow \Sigma_{b}^{0} D^{0} \ell^{+} \nu_{\ell}$ & $\frac{c_{5} V_{c d}^{*}}{\sqrt{2}}$ \\
\hline$\Omega_{c c b}^{+} \rightarrow \Xi_{c c}^{++} \ell^{-} \bar{\nu}_{\ell}$ & $c_{9} V_{u b}$ & $\Omega_{c c b}^{+} \rightarrow \Sigma_{b}^{-} D^{+} \ell^{+} \nu_{\ell}$ & $c_{5} V_{c d}^{*}$ \\
\hline$\Omega_{c c b}^{+} \rightarrow \Xi_{b c}^{0} \ell^{+} \nu_{\ell}$ & $c_{1} V_{c d}^{*}$ & $\Omega_{c c b}^{+} \rightarrow \Xi_{b}^{\prime 0} D^{0} \ell^{+} \nu_{\ell}$ & $\frac{c_{5} V_{c s}^{*}}{\sqrt{2}}$ \\
\hline$\Omega_{c c b}^{+} \rightarrow \Omega_{b c}^{0} \ell^{+} \nu_{\ell}$ & $c_{1} V_{c s}^{*}$ & $\Omega_{c c b}^{+} \rightarrow \Xi_{b}^{\prime-} D^{+} \ell^{+} \nu_{\ell}$ & $\frac{c_{5} V_{c s}^{*}}{\sqrt{2}}$ \\
\hline$\Omega_{c c b}^{+} \rightarrow \Xi_{b c}^{+} \pi^{-} \ell^{+} \nu_{\ell}$ & $c_{2} V_{c d}^{*}$ & $\Omega_{c c b}^{+} \rightarrow \Xi_{b}^{\prime-} D_{s}^{+} \ell^{+} \nu_{\ell}$ & $\frac{c_{5} V_{c d}^{*}}{\sqrt{2}}$ \\
\hline$\Omega_{c c b}^{+} \rightarrow \Xi_{b c}^{+} K^{-} \ell^{+} \nu_{\ell}$ & $c_{2} V_{c s}^{*}$ & $\Omega_{c c b}^{+} \rightarrow \Omega_{b}^{-} D_{s}^{+} \ell^{+} \nu_{\ell}$ & $c_{5} V_{c s}^{*}$ \\
\hline$\Omega_{c c b}^{+} \rightarrow \Xi_{b c}^{0} \pi^{0} \ell^{+} \nu_{\ell}$ & $-\frac{c_{2} V_{c d}^{*}}{\sqrt{2}}$ & $\Omega_{c c b}^{+} \rightarrow \Sigma_{c}^{+} B^{-} \ell^{+} \nu_{\ell}$ & $\frac{c_{6} V_{c d}^{*}}{\sqrt{2}}$ \\
\hline$\Omega_{c c b}^{+} \rightarrow \Xi_{b c}^{0} \bar{K}^{0} \ell^{+} \nu_{\ell}$ & $c_{2} V_{c s}^{*}$ & $\Omega_{c c b}^{+} \rightarrow \Sigma_{c}^{0} \bar{B}^{0} \ell^{+} \nu_{\ell}$ & $c_{6} V_{c d}^{*}$ \\
\hline$\Omega_{c c b}^{+} \rightarrow \Xi_{b c}^{0} \eta \ell^{+} \nu_{\ell}$ & $\frac{c_{2} V_{c d}^{*}}{\sqrt{6}}$ & $\Omega_{c c b}^{+} \rightarrow \Xi_{c}^{\prime+} B^{-} \ell^{+} \nu_{\ell}$ & $\frac{c_{6} V_{c s}^{*}}{\sqrt{2}}$ \\
\hline$\Omega_{c c b}^{+} \rightarrow \Omega_{b c}^{0} K^{0} \ell^{+} \nu_{\ell}$ & $c_{2} V_{c d}^{*}$ & $\Omega_{c c b}^{+} \rightarrow \Xi_{c}^{\prime 0} \bar{B}^{0} \ell^{+} \nu_{\ell}$ & $\frac{c_{6} V_{c s}^{*}}{\sqrt{2}}$ \\
\hline$\Omega_{c c b}^{+} \rightarrow \Omega_{b c}^{0} \eta \ell^{+} \nu_{\ell}$ & $-\sqrt{\frac{2}{3}} c_{2} V_{c s}^{*}$ & $\Omega_{c c b}^{+} \rightarrow \Xi_{c}^{\prime 0} \bar{B}_{s}^{0} \ell^{+} \nu_{\ell}$ & $\frac{c_{6} V_{c d}^{*}}{\sqrt{2}}$ \\
\hline$\Omega_{c c b}^{+} \rightarrow \Lambda_{b}^{0} D^{0} \ell^{+} \nu_{\ell}$ & $c_{3} V_{c d}^{*}$ & $\Omega_{c c b}^{+} \rightarrow \Omega_{c}^{0} \bar{B}_{s}^{0} \ell^{+} \nu_{\ell}$ & $c_{6} V_{c s}^{*}$ \\
\hline$\Omega_{c c b}^{+} \rightarrow \Xi_{b}^{0} D^{0} \ell^{+} \nu_{\ell}$ & $c_{3} V_{c s}^{*}$ & $\Omega_{c c b}^{+} \rightarrow \Xi_{c c}^{++} D^{0} \ell^{-} \bar{\nu}_{\ell}$ & $c_{8} V_{c b}$ \\
\hline$\Omega_{c c b}^{+} \rightarrow \Xi_{b}^{-} D^{+} \ell^{+} \nu_{\ell}$ & $c_{3} V_{c s}^{*}$ & $\Omega_{c c b}^{+} \rightarrow \Xi_{c c}^{+} D^{+} \ell^{-} \bar{\nu}_{\ell}$ & $c_{8} V_{c b}$ \\
\hline$\Omega_{c c b}^{+} \rightarrow \Xi_{b}^{-} D_{s}^{+} \ell^{+} \nu_{\ell}$ & $-c_{3} V_{c d}^{*}$ & $\Omega_{c c b}^{+} \rightarrow \Omega_{c c}^{+} D_{s}^{+} \ell^{-} \bar{\nu}_{\ell}$ & $c_{8} V_{c b}$ \\
\hline$\Omega_{c c b}^{+} \rightarrow \Lambda_{c}^{+} B^{-} \ell^{+} \nu_{\ell}$ & $c_{4} V_{c d}^{*}$ & $\Omega_{c c b}^{+} \rightarrow \Xi_{c c}^{++} \pi^{0} \ell^{-} \bar{\nu}_{\ell}$ & $\frac{c_{10} V_{u b}}{\sqrt{2}}$ \\
\hline$\Omega_{c c b}^{+} \rightarrow \Xi_{c}^{+} B^{-} \ell^{+} \nu_{\ell}$ & $c_{4} V_{c s}^{*}$ & $\Omega_{c c b}^{+} \rightarrow \Xi_{c c}^{++} \eta \ell^{-} \bar{\nu}_{\ell}$ & $\frac{c_{10} V_{u b}}{\sqrt{6}}$ \\
\hline$\Omega_{c c b}^{+} \rightarrow \Xi_{c}^{0} \bar{B}^{0} \ell^{+} \nu_{\ell}$ & $c_{4} V_{c s}^{*}$ & $\Omega_{c c b}^{+} \rightarrow \Xi_{c c}^{+} \pi^{+} \ell^{-} \bar{\nu}_{\ell}$ & $c_{10} V_{u b}$ \\
\hline$\Omega_{c c b}^{+} \rightarrow \Xi_{c}^{0} \bar{B}_{s}^{0} \ell^{+} \nu_{\ell}$ & $-c_{4} V_{c d}^{*}$ & $\Omega_{c c b}^{+} \rightarrow \Omega_{c c}^{+} K^{+} \ell^{-} \bar{\nu}_{\ell}$ & $c_{10} V_{u b}$ \\
\hline$\Omega_{c c b}^{+} \rightarrow \Sigma_{c}^{++} D^{0} \ell^{-} \bar{\nu}_{\ell}$ & $c_{12} V_{u b}$ & $\Omega_{c c b}^{+} \rightarrow \Lambda_{c}^{+} D^{+} \ell^{-} \bar{\nu}_{\ell}$ & $-c_{11} V_{u b}$ \\
\hline$\Omega_{c c b}^{+} \rightarrow \Sigma_{c}^{+} D^{+} \ell^{-} \bar{\nu}_{\ell}$ & $\frac{c_{12} V_{u b}}{\sqrt{2}}$ & $\Omega_{c c b}^{+} \rightarrow \Xi_{c}^{+} D_{s}^{+} \ell^{-} \bar{\nu}_{\ell}$ & $-c_{11} V_{u b}$ \\
\hline$\Omega_{c c b}^{+} \rightarrow \Xi_{c}^{\prime+} D_{s}^{+} \ell^{-} \bar{\nu}_{\ell}$ & 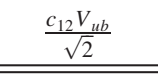 & & \\
\hline
\end{tabular}

Expanding the above equations, we will obtain the decay amplitudes given in Table IV.

\section{NONLEPTONIC $\boldsymbol{\Omega}_{c c c}$ DECAYS}

Nonleptonic charm-quark decays into light quarks are classified into three groups:
TABLE IV. Amplitudes for $\Omega_{c b b}$ semileptonic decay.

\begin{tabular}{|c|c|c|c|}
\hline Channel & Amplitude & Channel & Amplitude \\
\hline$\Omega_{c b b}^{0} \rightarrow \Omega_{c c b}^{+} \ell^{-} \bar{\nu}_{\ell}$ & $d_{1} V_{c b}$ & $\Omega_{c b b}^{0} \rightarrow \Xi_{c c}^{++} B^{-} \ell^{-} \bar{\nu}_{\ell}$ & $d_{2} V_{c b}$ \\
\hline$\Omega_{c b b}^{0} \rightarrow \Xi_{b c}^{+} \ell^{-} \bar{\nu}_{\ell}$ & $d_{4} V_{u b}$ & $\Omega_{c b b}^{0} \rightarrow \Xi_{c c}^{+} \bar{B}^{0} \ell^{-} \bar{\nu}_{\ell}$ & $d_{2} V_{c b}$ \\
\hline$\Omega_{c b b}^{0} \rightarrow \Xi_{b b}^{-} \ell^{+} \nu_{\ell}$ & $d_{10} V_{c d}^{*}$ & $\Omega_{c b b}^{0} \rightarrow \Omega_{c c}^{+} \bar{B}_{s}^{0} \ell^{-} \bar{\nu}_{\ell}$ & $d_{2} V_{c b}$ \\
\hline$\Omega_{c b b}^{0} \rightarrow \Omega_{b b}^{-} \ell^{+} \nu_{\ell}$ & $d_{10} V_{c s}^{*}$ & $\Omega_{c b b}^{0} \rightarrow \Xi_{b c}^{+} D^{0} \ell^{-} \bar{\nu}_{\ell}$ & $d_{3} V_{c b}$ \\
\hline$\Omega_{c b b}^{0} \rightarrow \Xi_{b b}^{0} \pi^{-} \ell^{+} \nu_{\ell}$ & $d_{11} V_{c d}^{*}$ & $\Omega_{c b b}^{0} \rightarrow \Xi_{b c}^{0} D^{+} \ell^{-} \bar{\nu}_{\ell}$ & $d_{3} V_{c b}$ \\
\hline$\Omega_{c b b}^{0} \rightarrow \Xi_{b b}^{0} K^{-} \ell^{+} \nu_{\ell}$ & $d_{11} V_{c s}^{*}$ & $\Omega_{c b b}^{0} \rightarrow \Omega_{b c}^{0} D_{s}^{+} \ell^{-} \bar{\nu}_{\ell}$ & $d_{3} V_{c b}$ \\
\hline$\Omega_{c b b}^{0} \rightarrow \Xi_{b b}^{-} \pi^{0} \ell^{+} \nu_{\ell}$ & $-\frac{d_{11} V_{c d}^{*}}{\sqrt{2}}$ & $\Omega_{c b b}^{0} \rightarrow \Xi_{b c}^{+} \pi^{0} \ell^{-} \bar{\nu}_{\ell}$ & $\frac{d_{5} V_{u b}}{\sqrt{2}}$ \\
\hline$\Omega_{c b b}^{0} \rightarrow \Xi_{b b}^{-} \bar{K}^{0} \ell^{+} \nu_{\ell}$ & $d_{11} V_{c s}^{*}$ & $\Omega_{c b b}^{0} \rightarrow \Xi_{b c}^{+} \eta \ell^{-} \bar{\nu}_{\ell}$ & $\frac{d_{5} V_{u b}}{\sqrt{6}}$ \\
\hline$\Omega_{c b b}^{0} \rightarrow \Xi_{b b}^{-} \eta \ell^{+} \nu_{\ell}$ & $\frac{d_{11} V_{c d}^{*}}{\sqrt{6}}$ & $\Omega_{c b b}^{0} \rightarrow \Xi_{b c}^{0} \pi^{+} \ell^{-} \bar{\nu}_{\ell}$ & $d_{5} V_{u b}$ \\
\hline$\Omega_{c b b}^{0} \rightarrow \Omega_{b b}^{-} K^{0} \ell^{+} \nu_{\ell}$ & $d_{11} V_{c d}^{*}$ & $\Omega_{c b b}^{0} \rightarrow \Omega_{b c}^{0} K^{+} \ell^{-} \bar{\nu}_{\ell}$ & $d_{5} V_{u b}$ \\
\hline$\Omega_{c b b}^{0} \rightarrow \Omega_{b b}^{-} \eta \ell^{+} \nu_{\ell}$ & $-\sqrt{\frac{2}{3}} d_{11} V_{c s}^{*}$ & $\Omega_{c b b}^{0} \rightarrow \Lambda_{b}^{0} D^{+} \ell^{-} \bar{\nu}_{\ell}$ & $-d_{6} V_{u b}$ \\
\hline$\Omega_{c b b}^{0} \rightarrow \Lambda_{b}^{0} B^{-} \ell^{+} \nu_{\ell}$ & $d_{12} V_{c d}^{*}$ & $\Omega_{c b b}^{0} \rightarrow \Xi_{b}^{0} D_{s}^{+} \ell^{-} \bar{\nu}_{\ell}$ & $-d_{6} V_{u b}$ \\
\hline$\Omega_{c b b}^{0} \rightarrow \Xi_{b}^{0} B^{-} \ell^{+} \nu_{\ell}$ & $d_{12} V_{c s}^{*}$ & $\Omega_{c b b}^{0} \rightarrow \Lambda_{c}^{+} \bar{B}^{0} \ell^{-} \bar{\nu}_{\ell}$ & $-d_{7} V_{u b}$ \\
\hline$\Omega_{c b b}^{0} \rightarrow \Xi_{b}^{-} \bar{B}^{0} \ell^{+} \nu_{\ell}$ & $d_{12} V_{c s}^{*}$ & $\Omega_{c b b}^{0} \rightarrow \Xi_{c}^{+} \bar{B}_{s}^{0} \ell^{-} \bar{\nu}_{\ell}$ & $-d_{7} V_{u b}$ \\
\hline$\Omega_{c b b}^{0} \rightarrow \Xi_{b}^{-} \bar{B}_{s}^{0} \ell^{+} \nu_{\ell}$ & $-d_{12} V_{c d}^{*}$ & $\Omega_{c b b}^{0} \rightarrow \Sigma_{b}^{+} D^{0} \ell^{-} \bar{\nu}_{\ell}$ & $d_{8} V_{u b}$ \\
\hline$\Omega_{c b b}^{0} \rightarrow \Sigma_{b}^{0} B^{-} \ell^{+} \nu_{\ell}$ & $\frac{d_{13} V_{c d}^{*}}{\sqrt{2}}$ & $\Omega_{c b b}^{0} \rightarrow \Sigma_{b}^{0} D^{+} \ell^{-} \bar{\nu}_{\ell}$ & $\frac{d_{8} V_{u b}}{\sqrt{2}}$ \\
\hline$\Omega_{c b b}^{0} \rightarrow \Sigma_{b}^{-} \bar{B}^{0} \ell^{+} \nu_{\ell}$ & $d_{13} V_{c d}^{*}$ & $\Omega_{c b b}^{0} \rightarrow \Xi_{b}^{\prime 0} D_{s}^{+} \ell^{-} \bar{\nu}_{\ell}$ & $\frac{d_{8} V_{u b}}{\sqrt{2}}$ \\
\hline$\Omega_{c b b}^{0} \rightarrow \Xi_{b}^{\prime 0} B^{-} \ell^{+} \nu_{\ell}$ & $\frac{d_{13} V_{c s}^{*}}{\sqrt{2}}$ & $\Omega_{c b b}^{0} \rightarrow \Sigma_{c}^{++} B^{-} \ell^{-} \bar{\nu}_{\ell}$ & $d_{9} V_{u b}$ \\
\hline$\Omega_{c b b}^{0} \rightarrow \Xi_{b}^{\prime-} \bar{B}^{0} \ell^{+} \nu_{\ell}$ & $\frac{d_{13} V_{c s}^{*}}{\sqrt{2}}$ & $\Omega_{c b b}^{0} \rightarrow \Sigma_{c}^{+} \bar{B}^{0} \ell^{-} \bar{\nu}_{\ell}$ & $\frac{d_{9} V_{u b}}{\sqrt{2}}$ \\
\hline$\Omega_{c b b}^{0} \rightarrow \Xi_{b}^{\prime-} \bar{B}_{s}^{0} \ell^{+}$ & $\frac{d_{13} V_{c d}^{*}}{\sqrt{2}}$ & $\Omega_{c b b}^{0} \rightarrow \Xi_{c}^{\prime+} \bar{B}_{s}^{0} \ell^{-} \bar{\nu}_{\ell}$ & $\frac{d_{9} V_{u b}}{\sqrt{2}}$ \\
\hline$\Omega_{c b b}^{0} \rightarrow \Omega_{b}^{-} \bar{B}_{s}^{0} \ell^{+} \nu_{\ell}$ & $d_{13} V_{c s}^{*}$ & & \\
\hline
\end{tabular}

$$
c \rightarrow s \bar{d} u, \quad c \rightarrow u \bar{d} d / \bar{s} s, \quad c \rightarrow d \bar{s} u
$$

Feynman diagrams for two-body decays induced by the $c \rightarrow s \bar{d} u$ are given in Fig. 4. In the first two panels, the final state contains a doubly charmed baryon and a light meson, while the last panel corresponds to decays into a charmed baryon and a charmed meson. The first panel is color allowed, while the last two panels are suppressed by $1 / N_{c}$.
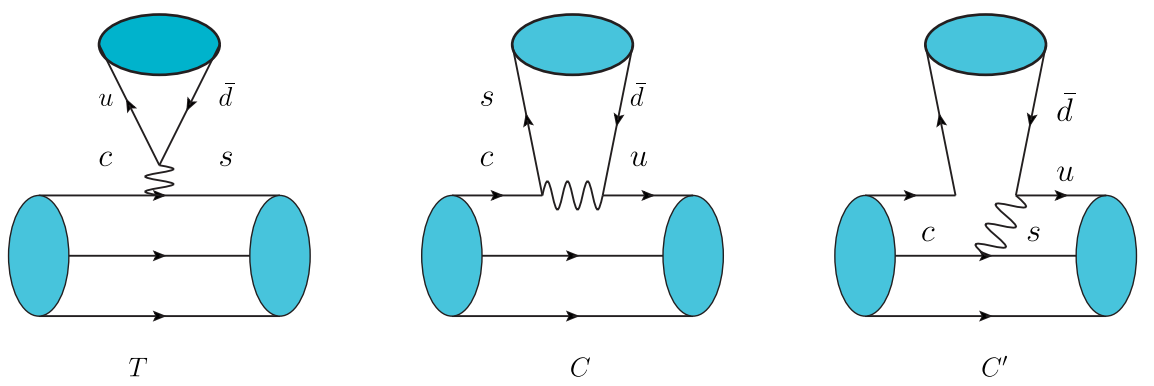

FIG. 4. Feynman diagrams for two-body decay modes induced by Cabibbo-allowed transitions. In the first two panels, the final state contains a doubly charmed baryon and a light meson, described by Eq. (53). The last panel corresponds to decays into a charmed baryon and a charmed meson. The first panel is color allowed, while the last two panels are suppressed by $1 / N_{c}$. 
TABLE V. Amplitudes for $\Omega_{c c c}$ decays into a doubly charmed baryon and a light meson. Cabibbo-allowed, singly Cabibbo-suppressed, and doubly Cabibbo-suppressed decay channels are included in this table and the following ones. The amplitudes $a_{i}$ 's are different than the ones in Table I.

\begin{tabular}{lccc}
\hline \hline Cabibbo-allowed & Amplitude & Singly Cabibbo-suppressed & Amplitude \\
$\Omega_{c c c}^{++} \rightarrow \Xi_{c c}^{++} \bar{K}^{0}$ & $\left(a_{2}-a_{1}\right) V_{u d} V_{c s}^{*}$ & $\Omega_{c c c}^{++} \rightarrow \Xi_{c c}^{++} \pi^{0}$ & $\frac{\left(a_{2}-a_{1}\right) V_{u s} V_{c s}^{*}}{\sqrt{2}}$ \\
$\Omega_{c c c}^{++} \rightarrow \Omega_{c c}^{+} \pi^{+}$ & $\left(a_{1}+a_{2}\right) V_{u d} V_{c s}^{*}$ & $\Omega_{c c c}^{++} \rightarrow \Xi_{c c}^{++} \eta$ & $\sqrt{\frac{3}{2}}\left(a_{1}-a_{2}\right) V_{u s} V_{c s}^{*}$ \\
Doubly Cabibbo-suppressed & Amplitude & $\Omega_{c c c}^{++} \rightarrow \Xi_{c c}^{+} \pi^{+}$ & $\left(a_{1}+a_{2}\right)\left(-V_{u s} V_{c s}^{*}\right)$ \\
$\Omega_{c c c}^{++} \rightarrow \Xi_{c c}^{++} K^{0}$ & $\left(a_{1}-a_{2}\right)\left(-V_{u s} V_{c d}^{*}\right)$ & $\Omega_{c c c}^{++} \rightarrow \Omega_{c c}^{+} K^{+}$ & $\left(a_{1}+a_{2}\right) V_{u s} V_{c s}^{*}$ \\
$\Omega_{c c c}^{++} \rightarrow \Xi_{c c}^{+} K^{+}$ & $\left(a_{1}+a_{2}\right) V_{u s} V_{c d}^{*}$ & & \\
\hline \hline
\end{tabular}

Penguin contributions in charm-quark decays are highly suppressed and thus are neglected in our analysis. Tree operators transform under the flavor SU(3) symmetry as $\mathbf{3} \otimes \overline{\mathbf{3}} \otimes \mathbf{3}=\mathbf{3} \oplus \mathbf{3} \oplus \overline{\mathbf{6}} \oplus \mathbf{1 5}$. For charmquark decays, the vector representation $H_{3}$ will vanish as an approximation. For the $c \rightarrow s u \bar{d}$ transition, we have

$$
\begin{gathered}
\left(H_{\overline{6}}\right)_{2}^{31}=-\left(H_{\overline{6}}\right)_{2}^{13}=V_{u d} V_{c s}^{*}, \\
\left(H_{15}\right)_{2}^{31}=\left(H_{15}\right)_{2}^{13}=V_{u d} V_{c s}^{*},
\end{gathered}
$$

while for the doubly Cabibbo-suppressed $c \rightarrow d u \bar{s}$ transition, we have

$$
\begin{gathered}
\left(H_{\overline{6}}\right)_{3}^{21}=-\left(H_{\overline{6}}\right)_{3}^{12}=V_{u s} V_{c d}^{*}, \\
\left(H_{15}\right)_{3}^{21}=\left(H_{15}\right)_{3}^{12}=V_{u s} V_{c d}^{*} .
\end{gathered}
$$

CKM matrix elements for $c \rightarrow u \bar{d} d$ and $c \rightarrow u \bar{s} s$ transitions are approximately equal in magnitude but different in sign. Here, we take $V_{u d} V_{c d}^{*}=-V_{u s} V_{c s}^{*}$, with both contributions, and one has the nonzero components:

$$
\begin{gathered}
\left(H_{\overline{6}}\right)_{3}^{31}=-\left(H_{\overline{6}}\right)_{3}^{13}=\left(H_{\overline{6}}\right)_{2}^{12}=-\left(H_{\overline{6}}\right)_{2}^{21}=V_{u s} V_{c s}^{*}, \\
\left(H_{15}\right)_{3}^{31}=\left(H_{15}\right)_{3}^{13}=-\left(H_{15}\right)_{2}^{12}=-\left(H_{15}\right)_{2}^{21}=V_{u s} V_{c s}^{*} .
\end{gathered}
$$

\section{A. Decays into a doubly charmed baryon and one (two) light meson(s)}

For decays into a doubly charmed baryon and a light meson, one may derive the effective Hamiltonian

$$
\begin{aligned}
\mathcal{H}_{\text {eff }}= & a_{1} \Omega_{c c c}\left(\bar{T}_{c c}\right)_{i}\left(M_{8}\right)_{j}^{k}\left(H_{\overline{6}}\right)_{k}^{i j} \\
& +a_{2} \Omega_{c c c}\left(\bar{T}_{c c}\right)_{i}\left(M_{8}\right)_{j}^{k}\left(H_{15}\right)_{k}^{i j},
\end{aligned}
$$

the Feynman diagrams of which are given in Fig. 4. It is necessary to stress that the above $\mathrm{SU}(3)$ independent amplitudes $a_{i}$ s are different than the ones in Eq. (25).
Expanding the above equations, we will obtain the decay amplitudes given in Table V. From Eq. (53) and Table V, one can see that there are two SU(3)-independent amplitudes, and thus there exist a few relations for the decay widths. These relations can be directly read off from Table V and can be examined by future experiments.

For the color-allowed decay channel, one may use the factorization approach to predict its decay widths. Using the form factors, we have the decay width

$$
\begin{aligned}
& \Gamma\left(\Omega_{c c c}^{++} \rightarrow \Omega_{c c}^{+} \pi^{+}\right) \\
& \quad=\frac{\sqrt{\lambda} G_{F}^{2}}{64 \pi m_{\Omega_{c c c}^{+}}^{3}}\left|V_{\mathrm{cs}} V_{u d}\right|^{2} f_{\pi}^{2}\left[\left|H_{t,-1 / 2}^{V, 1 / 2}\right|^{2}+\left|H_{t,-1 / 2}^{A, 1 / 2}\right|^{2}\right],
\end{aligned}
$$

where the $H_{i}$ s are helicity amplitudes:

$$
\begin{aligned}
& H_{t,-1 / 2}^{V, 1 / 2}=-\frac{\left(f_{1}+f_{2}-f_{4}\right) \sqrt{\lambda}}{\sqrt{6 m_{\pi}^{2}}}, \\
& H_{t,-1 / 2}^{A, 1 / 2}=\frac{\left(g_{1}-g_{2}+g_{4}\right) \hat{f}_{-}}{\sqrt{6 m_{\pi}^{2}}} .
\end{aligned}
$$

In the above equation, we used $m_{\Omega_{c c}^{+}}=m_{\Omega_{c c}^{+}} / m_{\Omega_{c c}^{+}}$, $m_{\pi}=m_{\pi} / m_{\Omega_{c c c}^{++}}$, and the abbreviations

$$
\begin{aligned}
\lambda \equiv & \lambda\left(m_{\Omega_{c c c}^{++}}^{2}, m_{\Omega_{c c}^{+}}^{2}, m_{\pi}^{2}\right)=\left(m_{\Omega_{c c c}^{++}}^{2}-m_{\Omega_{c c}^{+}}^{2}-m_{\pi}^{2}\right)^{2} \\
& -4 m_{\Omega_{c c}^{+}}^{2} m_{\pi}^{2}, \\
\lambda \equiv & \lambda\left(1, m_{\Omega_{c c}^{+}}^{2}, m_{\pi}^{2}\right)=\left(1-m_{\Omega_{c c}^{+}}^{2}-m_{\pi}^{2}\right)^{2}-4 m_{\Omega_{c c}^{+}}^{2} m_{\pi}^{2}, \\
\hat{f}_{-}= & \left(1-m_{\Omega_{c c}^{+}}\right)^{2}-m_{\pi}^{2} .
\end{aligned}
$$

Employing the same numerical inputs with the previous section, we obtain a very rough estimate of partial decay widths:

$$
\Gamma\left(\Omega_{c c c}^{++} \rightarrow \Omega_{c c}^{+} \pi^{+}\right)=6.20 \times 10^{-14} \mathrm{GeV} .
$$

Here, $f_{\pi}=0.13 \mathrm{GeV}$ is used [6]. With the NLO lifetime given in Eq. (13), we can derive the branching fractions: 
TABLE VI. Amplitudes for $\Omega_{c c c}$ decays into a doubly charmed baryon and two light mesons. The amplitude $b_{2}$ defined in Eq. (59) is not shown since it always accompanies $b_{1}$ in the form $b_{1}-b_{2}$.

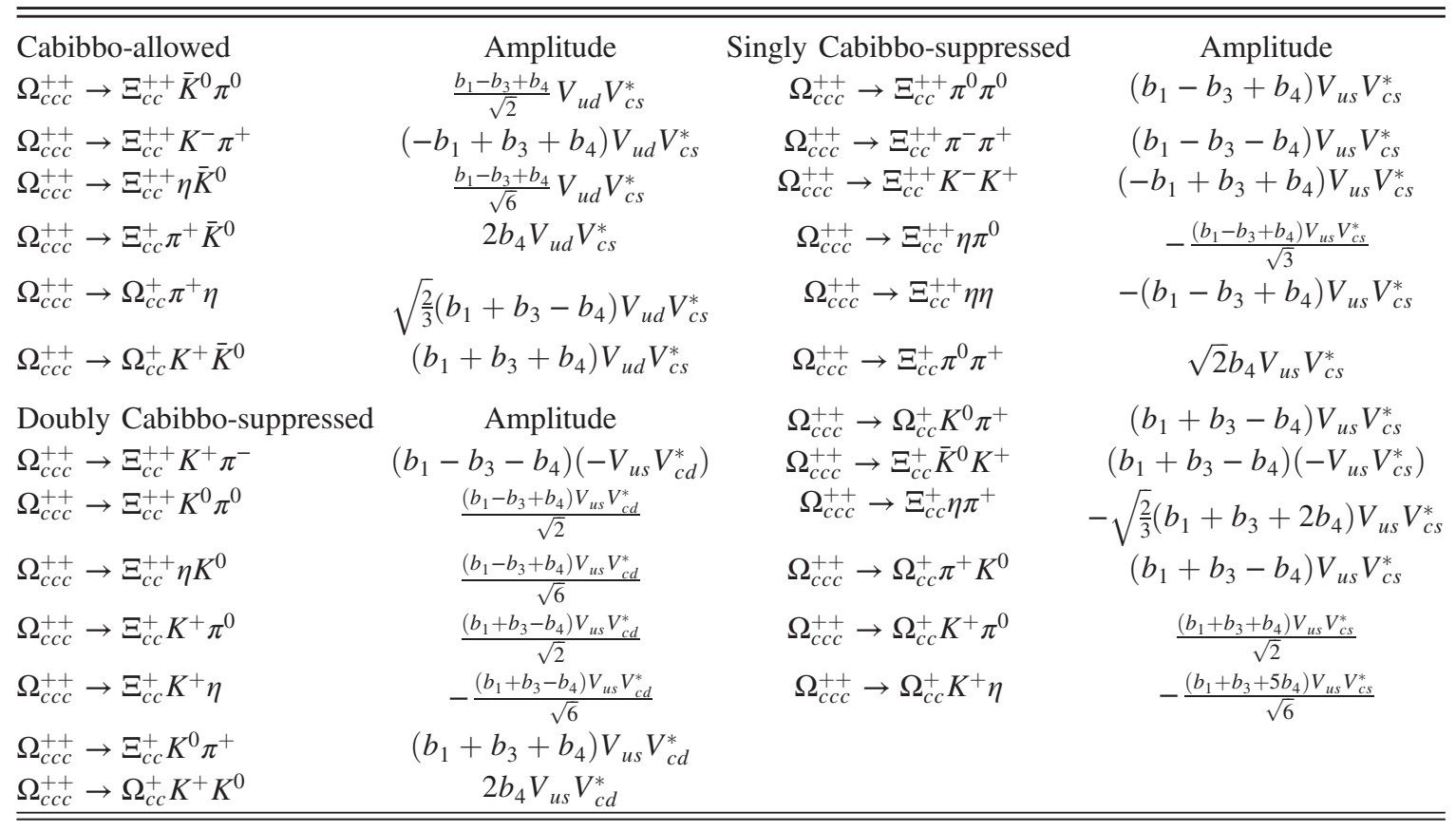

$$
\mathcal{B}\left(\Omega_{c c c}^{++} \rightarrow \Omega_{c c}^{+} \pi^{+}\right)=1.5 \%
$$

Such a large decay branching fraction indicates that this channel is very promising in future experimental searches.

For the reactions with one additional light meson in the final state, one has the Hamiltonian

$$
\begin{aligned}
\mathcal{H}_{\mathrm{eff}}= & b_{1} \Omega_{c c c}\left(\bar{T}_{c c}\right)_{i}\left(M_{8}\right)_{j}^{k}\left(M_{8}\right)_{l}^{j}\left(H_{6}\right)_{k}^{i l} \\
& +b_{2} \Omega_{c c c}\left(\bar{T}_{c c}\right)_{i}\left(M_{8}\right)_{j}^{i}\left(M_{8}\right)_{l}^{k}\left(H_{\overline{6}}\right)_{k}^{j l} \\
& +b_{3} \Omega_{c c c}\left(\bar{T}_{c c}\right)_{i}\left(M_{8}\right)_{j}^{k}\left(M_{8}\right)_{l}^{j}\left(H_{15}\right)_{k}^{i l} \\
& +b_{4} \Omega_{c c c}\left(\bar{T}_{c c}\right)_{i}\left(M_{8}\right)_{j}^{i}\left(M_{8}\right)_{l}^{k}\left(H_{15}\right)_{k}^{j l} .
\end{aligned}
$$

Expanding the above equation, we will obtain the decay amplitudes given in Table VI. The following remarks are in order: (i) From the expanded Hamiltonian, one can find that the amplitudes $b_{1}$ and $b_{2}$ always appear in the combination $b_{1}-b_{2}$. Thus, we have removed the amplitude $b_{2}$ in Table VI.

(ii) For channels with two identical particles, there is a factor $1 / 2$ in the decay width.

\section{B. Decays into a charmed baryon and a charmed meson}

For the two-body decays into a charmed baryon and a charmed meson, the effective Hamiltonian for the decays of $\Omega_{c c c}$ into a singly charmed baryon and a charmed meson is given as

$\mathcal{H}_{\mathrm{eff}}=c_{1} \Omega_{c c c}\left(\bar{T}_{\mathbf{c} \overline{\mathbf{3}}}\right)_{[i j]} \bar{D}^{k}\left(H_{\overline{6}}\right)_{k}^{i j}+c_{2} \Omega_{c c c}\left(\bar{T}_{\mathbf{c 6}}\right)_{[i j]} \bar{D}^{k}\left(H_{15}\right)_{k}^{i j}$

TABLE VII. Amplitudes for $\Omega_{c c c}$ decays into a singly charmed baryon and a charmed meson.

\begin{tabular}{lccc}
\hline \hline Cabibbo-allowed & Amplitude & Singly Cabibbo-suppressed & Amplitude \\
$\Omega_{c c c}^{++} \rightarrow \Xi_{c}^{+} D^{+}$ & $-2 c_{1} V_{u d} V_{c s}^{*}$ & $\Omega_{c c c}^{++} \rightarrow \Lambda_{c}^{+} D^{+}$ & $2 c_{1} V_{u s} V_{c s}^{*}$ \\
$\Omega_{c c c}^{++} \rightarrow \Xi_{c}^{\prime+} D^{+}$ & $\sqrt{2} c_{2} V_{u d} V_{c s}^{*}$ & $\Omega_{c c c}^{++} \rightarrow \Xi_{c}^{+} D_{s}^{+}$ & $-2 c_{1} V_{u s} V_{c s}^{*}$ \\
Doubly Cabibbo-suppressed & Amplitude & $\Omega_{c c c}^{++} \rightarrow \Xi_{c}^{\prime+} D_{s}^{+}$ & $\sqrt{2} c_{2} V_{u s} V_{c s}^{*}$ \\
$\Omega_{c c c}^{++} \rightarrow \Sigma_{c}^{+} D_{s}^{+}$ & $\sqrt{2} c_{2} V_{u s} V_{c d}^{*}$ & $\Omega_{c c c}^{++} \rightarrow \Sigma_{c}^{+} D^{+}$ & $-\sqrt{2} c_{2} V_{u s} V_{c s}^{*}$ \\
$\Omega_{c c c}^{++} \rightarrow \Lambda_{c}^{+} D_{s}^{+}$ & $-2 c_{1} V_{u s} V_{c d}^{*}$ & & \\
\hline \hline
\end{tabular}


TABLE VIII. Amplitudes for three-body $\Omega_{c c c}$ decays into a singly charmed baryon (antitriplet), D meson, and a light meson.

\begin{tabular}{|c|c|c|c|}
\hline Cabibbo-allowed & Amplitude & Singly Cabibbo-suppressed & Amplitude \\
\hline$\Omega_{c c c}^{++} \rightarrow \Lambda_{c}^{+} D^{+} \bar{K}^{0}$ & $\left(-d_{2}+d_{3}+d_{4}-d_{5}\right) V_{u d} V_{c s}^{*}$ & $\Omega_{c c c}^{++} \rightarrow \Lambda_{c}^{+} D^{0} \pi^{+}$ & $\left(2 d_{1}+d_{2}+d_{4}\right) V_{u s} V_{c s}^{*}$ \\
\hline$\Omega_{c c c}^{++} \rightarrow \Xi_{c}^{+} D^{0} \pi^{+}$ & $\left(-2 d_{1}-d_{2}-d_{4}\right) V_{u d} V_{c s}^{*}$ & $\Omega_{c c c}^{++} \rightarrow \Lambda_{c}^{+} D^{+} \pi^{0}$ & $-\frac{\left(2 d_{1}+d_{2}-d_{4}+2 d_{5}\right) V_{u s} S_{c s}^{*}}{\sqrt{2}}$ \\
\hline$\Omega_{c c c}^{++} \rightarrow \Xi_{c}^{+} D^{+} \pi^{0}$ & $\frac{2 d_{1}+d_{3}+d_{5}}{\sqrt{2}} V_{u d} V_{c s}^{*}$ & $\Omega_{c c c}^{++} \rightarrow \Lambda_{c}^{+} D^{+} \eta$ & $\frac{\left(2 d_{1}+3 d_{2}-2 d_{3}-3 d_{4}\right) V_{u s} V_{c s}^{*}}{\sqrt{6}}$ \\
\hline$\Omega_{c c c}^{++} \rightarrow \Xi_{c}^{+} D^{+} \eta$ & $-\frac{2 d_{1}+d_{3}-3 d_{5}}{\sqrt{6}} V_{u d} V_{c s}^{*}$ & $\Omega_{c c c}^{++} \rightarrow \Lambda_{c}^{+} D_{s}^{+} \bar{K}^{0}$ & $\left(2 d_{1}+d_{3}-d_{5}\right) V_{u s} V_{c s}^{*}$ \\
\hline$\Omega_{c c c}^{++} \rightarrow \Xi_{c}^{+} D_{s}^{+} \bar{K}^{0}$ & $\left(-2 d_{1}-d_{2}+d_{4}\right) V_{u d} V_{c s}^{*}$ & $\Omega_{c c c}^{++} \rightarrow \Xi_{c}^{+} D^{0} K^{+}$ & $\left(2 d_{1}+d_{2}+d_{4}\right)\left(-V_{u s} V_{c s}^{*}\right)$ \\
\hline$\Omega_{c c c}^{++} \rightarrow \Xi_{c}^{0} D^{+} \pi^{+}$ & $\left(-d_{2}+d_{3}-d_{4}+d_{5}\right) V_{u d} V_{c s}^{*}$ & $\Omega_{c c c}^{++} \rightarrow \Xi_{c}^{+} D^{+} K^{0}$ & $\left(2 d_{1}+d_{3}-d_{5}\right)\left(-V_{u s} V_{c s}^{*}\right)$ \\
\hline Doubly Cabibbo-suppressed & Amplitude & $\Omega_{c c c}^{++} \rightarrow \Xi_{c}^{0} D_{s}^{+} \pi^{+}$ & $\left(d_{2}-d_{3}+d_{4}-d_{5}\right)\left(-V_{u s} V_{c s}^{*}\right)$ \\
\hline$\Omega_{c c c}^{++} \rightarrow \Lambda_{c}^{+} D^{0} K^{+}$ & $\left(2 d_{1}+d_{2}+d_{4}\right)\left(-V_{u s} V_{c d}^{*}\right)$ & $\Omega_{c c c}^{++} \rightarrow \Xi_{c}^{+} D_{s}^{+} \pi^{0}$ & $\frac{\left(-d_{2}+d_{3}+d_{4}+d_{5}\right) V_{u s} V_{c s}^{*}}{\sqrt{2}}$ \\
\hline$\Omega_{c c c}^{++} \rightarrow \Lambda_{c}^{+} D^{+} K^{0}$ & $\left(2 d_{1}+d_{2}-d_{4}\right)\left(-V_{u s} V_{c d}^{*}\right)$ & $\Omega_{c c c}^{++} \rightarrow \Xi_{c}^{+} D_{s}^{+} \eta$ & $\frac{\left(4 d_{1}+3 d_{2}-d_{3}-3 d_{4}+3 d_{5}\right) V_{u s} V_{c s}^{*}}{\sqrt{6}}$ \\
\hline$\Omega_{c c c}^{++} \rightarrow \Lambda_{c}^{+} D_{s}^{+} \pi^{0}$ & $\sqrt{2} d_{5} V_{u s} V_{c d}^{*}$ & $\Omega_{c c c}^{++} \rightarrow \Xi_{c}^{0} D^{+} K^{+}$ & $\left(d_{2}-d_{3}+d_{4}-d_{5}\right)\left(-V_{u s} V_{c s}^{*}\right)$ \\
\hline$\Omega_{c c c}^{++} \rightarrow \Lambda_{c}^{+} D_{s}^{+} \eta$ & $\sqrt{\frac{2}{3}}\left(2 d_{1}+d_{3}\right) V_{u s} V_{c d}^{*}$ & & \\
\hline$\Omega_{c c c}^{++} \rightarrow \Xi_{c}^{+} D_{s}^{+} K^{0}$ & $\left(d_{2}-d_{3}-d_{4}+d_{5}\right)\left(-V_{u s} V_{c d}^{*}\right)$ & & \\
\hline$\underline{\Omega_{c c c}^{++} \rightarrow \Xi_{c}^{0} D_{s}^{+} K^{+}}$ & $\left(d_{2}-d_{3}+d_{4}-d_{5}\right) V_{u s} V_{c d}^{*}$ & & \\
\hline
\end{tabular}

TABLE IX. Amplitudes for three-body $\Omega_{c c c}$ decays into a singly charmed baryon (sextet), D meson, and a light meson.

\begin{tabular}{|c|c|c|c|}
\hline $\begin{array}{l}\text { Cabibbo-allowed } \\
\Omega_{c c c}^{++} \rightarrow \Sigma_{c}^{++} D^{0} \bar{K}^{0}\end{array}$ & $\begin{array}{c}\text { Amplitude } \\
\left(e_{2}-e_{4}\right) V_{u d} V_{c s}^{*}\end{array}$ & $\begin{array}{c}\text { Singly Cabibbo-suppressed } \\
\Omega_{c c c}^{++} \rightarrow \Sigma_{c}^{++} D^{0} \pi^{0}\end{array}$ & $\begin{array}{l}\text { Amplitude } \\
\underline{\left(e_{2}-e_{4}\right) V_{u s} V_{c s}^{*}}\end{array}$ \\
\hline$\Omega_{c c c}^{++} \rightarrow \Sigma_{c}^{++} D^{+} K^{-}$ & $\left(e_{3}-e_{5}\right) V_{u d} V_{c s}^{*}$ & $\Omega_{c c c}^{++} \rightarrow \Sigma_{c}^{++} D^{0} \eta$ & $-\sqrt{\frac{3}{2}}\left(e_{2}-e_{4}\right) V_{u s} V_{c s}^{*}$ \\
\hline$\Omega_{c c c}^{++} \rightarrow \Sigma_{c}^{+} D^{+} \bar{K}^{0}$ & $\frac{e_{2}+e_{3}-e_{4}-e_{5}}{\sqrt{2}} V_{u d} V_{c s}^{*}$ & $\Omega_{c c c}^{++} \rightarrow \Sigma_{c}^{++} D^{+} \pi^{-}$ & $\left(e_{5}-e_{3}\right) V_{u s} V_{c s}^{*}$ \\
\hline$\Omega_{c c c}^{++} \rightarrow \Xi_{c}^{\prime+} D^{0} \pi^{+}$ & $\frac{2 e_{1}+e_{2}+e_{4}}{\sqrt{2}} V_{u d} V_{c s}^{*}$ & $\Omega_{c c c}^{++} \rightarrow \Sigma_{c}^{++} D_{s}^{+} K^{-}$ & $\left(e_{3}-e_{5}\right) V_{u s} V_{c s}^{*}$ \\
\hline$\Omega_{c c c}^{++} \rightarrow \Xi_{c}^{\prime+} D^{+} \pi^{0}$ & $\frac{1}{2}\left(-2 e_{1}+e_{3}+e_{5}\right) V_{u d} V_{c s}^{*}$ & $\Omega_{c c c}^{++} \rightarrow \Sigma_{c}^{+} D^{0} \pi^{+}$ & $-\frac{\left(2 e_{1}+e_{2}+e_{4}\right) V_{u s} V_{c s}^{*}}{\sqrt{2}}$ \\
\hline$\Omega_{c c c}^{++} \rightarrow \Xi_{c}^{\prime+} D^{+} \eta$ & $\frac{2 e_{1}-e_{3}+3 e_{5}}{2 \sqrt{3}} V_{u d} V_{c s}^{*}$ & $\Omega_{c c c}^{++} \rightarrow \Sigma_{c}^{+} D^{+} \pi^{0}$ & $\frac{1}{2}\left(2 e_{1}+e_{2}-e_{4}-2 e_{5}\right) V_{u s} V_{c s}^{*}$ \\
\hline$\Omega_{c c c}^{++} \rightarrow \Xi_{c}^{\prime+} D_{s}^{+} \bar{K}^{0}$ & $\frac{2 e_{1}+e_{2}-e_{4}}{\sqrt{2}} V_{u d} V_{c s}^{*}$ & $\Omega_{c c c}^{++} \rightarrow \Sigma_{c}^{+} D^{+} \eta$ & $-\frac{\left(2 e_{1}+3 e_{2}+2 e_{3}-3 e_{4}\right) V_{u s} V_{c s}^{*}}{2 \sqrt{3}}$ \\
\hline$\Omega_{c c c}^{++} \rightarrow \Xi_{c}^{\prime 0} D^{+} \pi^{+}$ & $\frac{e_{2}+e_{3}+e_{4}+e_{5}}{\sqrt{2}} V_{u d} V_{c S}^{*}$ & $\Omega_{c c c}^{++} \rightarrow \Sigma_{c}^{+} D_{s}^{+} \bar{K}^{0}$ & $-\frac{\left(2 e_{1}-e_{3}+e_{5}\right) V_{u s} V_{c s}^{*}}{\sqrt{2}}$ \\
\hline$\Omega_{c c c}^{++} \rightarrow \Omega_{c}^{0} D^{+} K^{+}$ & $\left(e_{3}+e_{5}\right) V_{u d} V_{c s}^{*}$ & $\Omega_{c c c}^{++} \rightarrow \Sigma_{c}^{0} D^{+} \pi^{+}$ & $\left(e_{2}+e_{3}+e_{4}+e_{5}\right)\left(-V_{u s} V_{c s}^{*}\right)$ \\
\hline$\Omega_{c c c}^{++} \rightarrow \Omega_{c}^{0} D_{s}^{+} \pi^{+}$ & $\left(e_{2}+e_{4}\right) V_{u d} V_{c s}^{*}$ & $\Omega_{c c c}^{++} \rightarrow \Xi_{c}^{\prime+} D^{0} K^{+}$ & $\frac{\left(2 e_{1}+e_{2}+e_{4}\right) V_{u s} V_{c s}^{*}}{\sqrt{2}}$ \\
\hline Doubly Cabibbo-suppressed & Amplitude & $\Omega_{c c c}^{++} \rightarrow \Omega_{c}^{0} D_{s}^{+} K^{+}$ & $\left(e_{2}+e_{3}+e_{4}+e_{5}\right) V_{u s} V_{c s}^{*}$ \\
\hline$\Omega_{c c c}^{++} \rightarrow \Sigma_{c}^{++} D^{0} K^{0}$ & $\left(e_{2}-e_{4}\right) V_{u s} V_{c d}^{*}$ & $\Omega_{c c c}^{++} \rightarrow \Xi_{c}^{+} D^{+} K^{0}$ & $\frac{\left(2 e_{1}-e_{3}+e_{5}\right) V_{u s} V_{c s}^{*}}{\sqrt{2}}$ \\
\hline$\Omega_{c c c}^{++} \rightarrow \Sigma_{c}^{++} D_{s}^{+} \pi^{-}$ & $\left(e_{3}-e_{5}\right) V_{u s} V_{c d}^{*}$ & $\Omega_{c c c}^{++} \rightarrow \Xi_{c}^{\prime+} D_{s}^{+} \pi^{0}$ & $\frac{1}{2}\left(e_{2}+e_{3}-e_{4}+e_{5}\right) V_{u s} V_{c s}^{*}$ \\
\hline$\Omega_{c c c}^{++} \rightarrow \Sigma_{c}^{+} D^{0} K^{+}$ & $\frac{\left(2 e_{1}+e_{2}+e_{4}\right) V_{u s} V_{c d}^{*}}{\sqrt{2}}$ & $\Omega_{c c c}^{++} \rightarrow \Xi_{c}^{\prime+} D_{s}^{+} \eta$ & $-\frac{\left(4 e_{1}+3 e_{2}+e_{3}-3 e_{4}-3 e_{5}\right) V_{u s} V_{c s}^{*}}{2 \sqrt{3}}$ \\
\hline$\Omega_{c c c}^{++} \rightarrow \Sigma_{c}^{+} D^{+} K^{0}$ & $\frac{\left(2 e_{1}+e_{2}-e_{4}\right) V_{u s} V_{c d}^{*}}{\sqrt{2}}$ & $\Omega_{c c c}^{++} \rightarrow \Xi_{c}^{\prime 0} D^{+} K^{+}$ & $\frac{\left(e_{2}-e_{3}+e_{4}-e_{5}\right) V_{u s} V_{c s}^{*}}{\sqrt{2}}$ \\
\hline$\Omega_{c c c}^{++} \rightarrow \Sigma_{c}^{+} D_{s}^{+} \pi^{0}$ & $e_{5} V_{u s} V_{c d}^{*}$ & $\Omega_{c c c}^{++} \rightarrow \Xi_{c}^{\prime 0} D_{s}^{+} \pi^{+}$ & $-\frac{\left(e_{2}-e_{3}+e_{4}-e_{5}\right) V_{u s} V_{c s}^{*}}{\sqrt{2}}$ \\
\hline$\Omega_{c c c}^{++} \rightarrow \Sigma_{c}^{+} D_{s}^{+} \eta$ & $\frac{\left(e_{3}-2 e_{1}\right) V_{u s} V_{c d}^{*}}{\sqrt{3}}$ & & \\
\hline$\Omega_{c c c}^{++} \rightarrow \Sigma_{c}^{0} D^{+} K^{+}$ & $\left(e_{2}+e_{4}\right) V_{u s} V_{c d}^{*}$ & & \\
\hline$\Omega_{c c c}^{++} \rightarrow \Sigma_{c}^{0} D_{s}^{+} \pi^{+}$ & $\left(e_{3}+e_{5}\right) V_{u s} V_{c d}^{*}$ & & \\
\hline$\Omega_{c c c}^{++} \rightarrow \Xi_{c}^{\prime+} D_{s}^{+} K^{0}$ & $\frac{\left(e_{2}+e_{3}-e_{4}-e_{5}\right) V_{u s} V_{c d}^{*}}{\sqrt{2}}$ & & \\
\hline$\Omega_{c c c}^{++} \rightarrow \Xi_{c}^{\prime 0} D_{s}^{+} K^{+}$ & $\frac{\left(e_{2}+e_{3}+e_{4}+e_{5}\right) V_{u s} V_{c d}^{*}}{\sqrt{2}}$ & & \\
\hline
\end{tabular}


The Feynman diagram is shown in the last panel of Fig. 4, and decay amplitudes are collected in Table VII.

The three-body decays of $\Omega_{c c c}$ can involve an additional light meson in the final state. For the modes with an antitriplet baryon, we have

$$
\begin{aligned}
\mathcal{H}_{\mathrm{eff}}= & d_{1} \Omega_{c c c}\left(\bar{T}_{\mathbf{c} \overline{\mathbf{3}}}\right)_{[i j]} \bar{D}^{k}\left(M_{8}\right)_{k}^{l}\left(H_{\overline{6}}\right)_{l}^{i j} \\
& +d_{2} \Omega_{c c c}\left(\bar{T}_{\mathbf{c} \overline{\mathbf{3}}}\right)_{[i j]} \bar{D}^{j}\left(M_{8}\right)_{k}^{l}\left(H_{\overline{6}}\right)_{l}^{i k} \\
& +d_{3} \Omega_{c c c}\left(\bar{T}_{\mathbf{c} \overline{\mathbf{3}}}\right)_{[i j]} \bar{D}^{l}\left(M_{8}\right)_{k}^{i}\left(H_{\overline{6}}\right)_{l}^{j k} \\
& +d_{4} \Omega_{c c c}\left(\bar{T}_{\mathbf{c} \overline{\mathbf{3}})_{[i j]}} \bar{D}^{j}\left(M_{8}\right)_{k}^{l}\left(H_{15}\right)_{l}^{i k}\right. \\
& +d_{5} \Omega_{c c c}\left(\bar{T}_{\mathbf{c} \overline{\mathbf{3}}}\right)_{[i j]} \bar{D}^{l}\left(M_{8}\right)_{k}^{i}\left(H_{15}\right)_{l}^{j k},
\end{aligned}
$$

while the effective Hamiltonian for a sextet baryon is constructed as

$$
\begin{aligned}
\mathcal{H}_{\mathrm{eff}}= & e_{1} \Omega_{c c c}\left(\bar{T}_{\mathbf{c 6}}\right)_{\{i j\}} \bar{D}^{k}\left(M_{8}\right)_{k}^{l}\left(H_{15}\right)_{l}^{i j} \\
& +e_{2} \Omega_{c c c}\left(\bar{T}_{\mathbf{c 6}}\right)_{\{i j\}} \bar{D}^{j}\left(M_{8}\right)_{k}^{l}\left(H_{15}\right)_{l}^{i k} \\
& +e_{3} \Omega_{c c c}\left(\bar{T}_{\mathbf{c 6}}\right)_{\{i j\}} \bar{D}^{l}\left(M_{8}\right)_{k}^{i}\left(H_{15}\right)_{l}^{j k} \\
& +e_{4} \Omega_{c c c}\left(\bar{T}_{\mathbf{c 6}}\right)_{\{i j\}} \bar{D}^{j}\left(M_{8}\right)_{k}^{l}\left(H_{\overline{6}}\right)_{l}^{i k} \\
& +e_{5} \Omega_{c c c}\left(\bar{T}_{\mathbf{c 6}}\right)_{\{i j\}} \bar{D}^{l}\left(M_{8}\right)_{k}^{i}\left(H_{\overline{6}}\right)_{l}^{j k} .
\end{aligned}
$$

Expanding the above equations, we will obtain the decay amplitudes given in Table VIII for antitriplet baryon and in Table IX for the sextet baryon.

\section{NONLEPTONIC $\Omega_{b b b}^{-}$DECAYS}

For the bottom-quark decay, there are four kinds of quark-level transitions,

$$
\begin{aligned}
b & \rightarrow c \bar{c} d / s, & b & \rightarrow c \bar{u} d / s, \\
b & \rightarrow u \bar{c} d / s, & b & \rightarrow q \bar{q} q,
\end{aligned}
$$

which will be studied in order.

$$
\text { A. } b \rightarrow c \bar{c} d / s
$$

\section{Decays into a charmonium $J / \psi$}

Such decays will have the same topology with the $b \rightarrow$ $s \ell^{+} \ell^{-}$decays. The transition operator $b \rightarrow c \bar{c} d / s$ can form an $\mathrm{SU}(3)$ triplet,

$$
\begin{aligned}
\mathcal{H}_{\text {eff }}= & f_{1} \Omega_{b b b}\left(\bar{T}_{b b}\right)_{i}\left(H_{3}\right)^{i} J / \psi \\
& +f_{2} \Omega_{b b b}\left(\bar{T}_{b b}\right)_{i}\left(M_{8}\right)_{j}^{i}\left(H_{3}\right)^{j} J / \psi \\
& +f_{3} \Omega_{b b b}\left(\bar{T}_{b \overline{3}}\right)_{[i j]} \bar{B}^{i}\left(H_{3}\right)^{j} J / \psi \\
& +f_{4} \Omega_{b b b}\left(\bar{T}_{b 6}\right)_{\{i j\}} \bar{B}^{i}\left(H_{3}\right)^{j} J / \psi,
\end{aligned}
$$

with $\left(H_{3}\right)_{2}=V_{c d}^{*}$ and $\left(H_{3}\right)_{3}=V_{c s}^{*}$. Decay amplitudes for different channels are obtained by expanding the above Hamiltonian and are collected in Table X.
TABLE X. Amplitudes for $\Omega_{b b b}$ decays into a $J / \psi$ and a doubly bottom baryon or two bottom hadrons.

\begin{tabular}{lccc}
\hline \hline Channel & Amplitude & Channel & Amplitude \\
\hline$\Omega_{b b b}^{-} \rightarrow \Xi_{b b}^{-} J / \psi$ & $f_{1} V_{c d}^{*}$ & $\Omega_{b b b}^{-} \rightarrow \Lambda_{b}^{0} B^{-} J / \psi$ & $f_{3} V_{c d}^{*}$ \\
$\Omega_{b b b}^{-} \rightarrow \Omega_{b b}^{-} J / \psi$ & $f_{1} V_{c s}^{*}$ & $\Omega_{b b b}^{-} \rightarrow \Xi_{b}^{0} B^{-} J / \psi$ & $f_{3} V_{c s}^{*}$ \\
$\Omega_{b b b}^{-} \rightarrow \Xi_{b b}^{0} \pi^{-} J / \psi$ & $f_{2} V_{c d}^{*}$ & $\Omega_{b b b}^{-} \rightarrow \Xi_{b}^{-} \bar{B}^{0} J / \psi$ & $f_{3} V_{c s}^{*}$ \\
$\Omega_{b b b}^{-} \rightarrow \Xi_{b b}^{0} K^{-} J / \psi$ & $f_{2} V_{c s}^{*}$ & $\Omega_{b b b}^{-} \rightarrow \Xi_{b}^{-} \bar{B}_{s}^{0} J / \psi$ & $-f_{3} V_{c d}^{*}$ \\
$\Omega_{b b b}^{-} \rightarrow \Xi_{b b}^{-} \pi^{0} J / \psi$ & $-\frac{f_{2} V_{c d}^{*}}{\sqrt{2}}$ & $\Omega_{b b b}^{-} \rightarrow \Sigma_{b}^{0} B^{-} J / \psi$ & $\frac{f_{4} V_{c d}^{*}}{\sqrt{2}}$ \\
$\Omega_{b b b}^{-} \rightarrow \Xi_{b b}^{-} \bar{K}^{0} J / \psi$ & $f_{2} V_{c s}^{*}$ & $\Omega_{b b b}^{-} \rightarrow \Sigma_{b}^{-} \bar{B}^{0} J / \psi$ & $f_{4} V_{c d}^{*}$ \\
$\Omega_{b b b}^{-} \rightarrow \Xi_{b b}^{-} \eta J / \psi$ & $\frac{f_{2} V_{c d}^{*}}{\sqrt{6}}$ & $\Omega_{b b b}^{-} \rightarrow \Xi_{b}^{\prime 0} B^{-} J / \psi$ & $\frac{f_{4} V_{c s}^{*}}{\sqrt{2}}$ \\
$\Omega_{b b b}^{-} \rightarrow \Omega_{b b}^{-} K^{0} J / \psi$ & $f_{2} V_{c d}^{*}$ & $\Omega_{b b b}^{-} \rightarrow \Xi_{b}^{\prime-} \bar{B}^{0} J / \psi$ & $\frac{f_{4} V_{c s}^{*}}{\sqrt{2}}$ \\
$\Omega_{b b b}^{-} \rightarrow \Omega_{b b}^{-} \eta J / \psi$ & $-\sqrt{\frac{2}{3}} f_{2} V_{c s}^{*}$ & $\Omega_{b b b}^{-} \rightarrow \Xi_{b}^{\prime-} \bar{B}_{s}^{0} J / \psi$ & $\frac{f_{4} V_{c d}^{*}}{\sqrt{2}}$ \\
& & $\Omega_{b b b}^{-} \rightarrow \Omega_{b}^{-} \bar{B}_{s}^{0} J / \psi$ & $f_{4} V_{c s}^{*}$ \\
\hline \hline
\end{tabular}

\section{Decays into a triply heavy baryon cbb plus an anticharmed meson}

The $b \rightarrow c \bar{c} d / s$ transition can lead to another type of effective Hamiltonian,

$\mathcal{H}_{\text {eff }}=g_{1} \Omega_{b b b} \bar{\Omega}_{c b b} D_{i}\left(H_{3}\right)^{i}+g_{2} \Omega_{b b b} \bar{\Omega}_{c b b} D_{j}\left(M_{8}\right)_{i}^{j}\left(H_{3}\right)^{i}$,

which corresponds to the decays into triply heavy baryon $\Omega_{c b b}$ plus an anticharmed meson. Decay amplitudes for different channels are obtained by expanding the above Hamiltonian and are collected in Table XI.

\section{B. $b \rightarrow c \bar{u} d / s$ transition}

\section{Decays into a triply heavy baryon cbb plus light mesons}

The operator to produce a charm quark from the $b$-quark decay, $\bar{c} b \bar{q} u$, is given by

$$
\mathcal{H}_{\text {e.w. }}=\frac{G_{F}}{\sqrt{2}} V_{c b} V_{u q}^{*}\left[C_{1} O_{1}^{\bar{c} u}+C_{2} O_{2}^{\bar{c} u}\right]+\text { H.c. }
$$

TABLE XI. Amplitudes for $\Omega_{b b b}$ decays into $\Omega_{c b b}$ and an anticharmed meson.

\begin{tabular}{lccc}
\hline \hline Channel & Amplitude & Channel & Amplitude \\
\hline$\Omega_{b b b}^{-} \rightarrow \Omega_{c b b}^{0} D^{-}$ & $g_{1} V_{c d}^{*}$ & $\Omega_{b b b}^{-} \rightarrow \Omega_{c b b}^{0} D_{s}^{-}$ & $g_{1} V_{c s}^{*}$ \\
$\Omega_{b b b}^{-} \rightarrow \Omega_{c b b}^{0} \bar{D}^{0} \pi^{-}$ & $g_{2} V_{c d}^{*}$ & $\Omega_{b b b}^{-} \rightarrow \Omega_{c b b}^{0} \bar{D}^{0} K^{-}$ & $g_{2} V_{c s}^{*}$ \\
$\Omega_{b b b}^{-} \rightarrow \Omega_{c b b}^{0} D^{-} \pi^{0}$ & $-\frac{g_{2} V_{c d}^{*}}{\sqrt{2}}$ & $\Omega_{b b b}^{-} \rightarrow \Omega_{c b b}^{0} D^{-} \bar{K}^{0}$ & $g_{2} V_{c s}^{*}$ \\
$\Omega_{b b b}^{-} \rightarrow \Omega_{c b b}^{0} D^{-} \eta$ & $\frac{g_{2} V_{c d}^{*}}{\sqrt{6}}$ & $\Omega_{b b b}^{-} \rightarrow \Omega_{c b b}^{0} D_{s}^{-} K^{0}$ & $g_{2} V_{c d}^{*}$ \\
$\Omega_{b b b}^{-} \rightarrow \Omega_{c b b}^{0} D_{s}^{-} \eta$ & $-\sqrt{\frac{2}{3}} g_{2} V_{c s}^{*}$ & & \\
\hline \hline
\end{tabular}


with

$$
\begin{aligned}
& O_{1}^{\bar{c} u}=\bar{q}^{i} \gamma^{\mu}\left(1-\gamma_{5}\right) u^{j} \bar{c}^{j} \gamma_{\mu}\left(1-\gamma_{5}\right) b^{i}, \\
& O_{2}^{\bar{c} u}=\bar{q} \gamma^{\mu}\left(1-\gamma_{5}\right) u \bar{c} \gamma_{\mu}\left(1-\gamma_{5}\right) b .
\end{aligned}
$$

The light quarks in this effective Hamiltonian form an octet with the nonzero entry

$$
\left(H_{\mathbf{8}}\right)_{1}^{2}=V_{c b} V_{u d}^{*}
$$

for the $b \rightarrow c \bar{u} d$ transition and $\left(H_{\mathbf{8}}\right)_{1}^{3}=V_{c b} V_{u s}^{*}$ for the $b \rightarrow c \bar{u} s$ transition,

$$
\mathcal{H}_{\mathrm{eff}}=h_{1} \Omega_{b b b} \bar{\Omega}_{c b b} M_{i}^{j}\left(H_{8}\right)_{j}^{i}+h_{2} \Omega_{b b b} \bar{\Omega}_{c b b} M_{k}^{j} M_{i}^{k}\left(H_{8}\right)_{j}^{i} .
$$

Decay amplitudes for different channels are obtained by expanding the above Hamiltonian and are collected in Table XII.
TABLE XII. Amplitudes for $\Omega_{b b b}$ decays into $\Omega_{c b b}$ and light meson(s).

\begin{tabular}{lccc}
\hline \hline Channel & Amplitude & Channel & Amplitude \\
\hline$\Omega_{b b b}^{-} \rightarrow \Omega_{b b c}^{0} \pi^{-}$ & $h_{1} V_{c b} V_{u d}^{*}$ & $\Omega_{b b b}^{-} \rightarrow \Omega_{b b c}^{0} K^{-}$ & $h_{1} V_{c b} V_{u s}^{*}$ \\
$\Omega_{b b b}^{-} \rightarrow \Omega_{b b c}^{0} \pi^{0} K^{-}$ & $\frac{h_{2} V_{c b} V_{u s}^{*}}{\sqrt{2}}$ & $\Omega_{b b b}^{-} \rightarrow \Omega_{b b c}^{0} \pi^{-} \eta$ & $\sqrt{\frac{2}{3}} h_{2} V_{c b} V_{u d}^{*}$ \\
$\Omega_{b b b}^{-} \rightarrow \Omega_{b b c}^{0} \pi^{-} \bar{K}^{0}$ & $h_{2} V_{c b} V_{u s}^{*}$ & $\Omega_{b b b}^{-} \rightarrow \Omega_{b b c}^{0} K^{0} K^{-}$ & $h_{2} V_{c b} V_{u d}^{*}$ \\
$\Omega_{b b b}^{-} \rightarrow \Omega_{b b c}^{0} K^{-} \eta$ & $-\frac{h_{2} V_{c b} V_{u s s}^{*}}{\sqrt{6}}$ & & \\
\hline \hline
\end{tabular}

\section{Decays into a charmed meson plus a doubly bottom baryon or two bottom hadrons}

If the $c \bar{c}$ are separated, then the final state could be a doubly bottom baryon and a charmed meson. The threebody modes can also include decays into a bottom baryon, a bottom meson, and a charmed meson. Thus, one can have the effective Hamiltonian

$$
\begin{aligned}
\mathcal{H}_{\mathrm{eff}}= & j_{1} \Omega_{b b b}\left(\bar{T}_{b b}\right)_{i} \bar{D}^{j}\left(H_{8}\right)_{j}^{i}+j_{2} \Omega_{b b b}\left(\bar{T}_{b b}\right)_{k} \bar{D}^{k}\left(M_{8}\right)_{i}^{j}\left(H_{8}\right)_{j}^{i}+j_{3} \Omega_{b b b}\left(\bar{T}_{b b}\right)_{i} \bar{D}^{k}\left(M_{8}\right)_{k}^{j}\left(H_{8}\right)_{j}^{i}+j_{4} \Omega_{b b b}\left(\bar{T}_{b b}\right)_{i} \bar{D}^{k}\left(M_{8}\right)_{j}^{i}\left(H_{8}\right)_{k}^{j} \\
& +j_{5} \Omega_{b b b}\left(\bar{T}_{b \overline{3}}\right)_{[i k]} \bar{B}^{j} \bar{D}^{k}\left(H_{8}\right)_{j}^{i}+j_{6} \Omega_{b b b}\left(\bar{T}_{b \overline{3}}\right)_{[i k]} \bar{B}^{k} \bar{D}^{j}\left(H_{8}\right)_{j}^{i}+j_{7} \Omega_{b b b}\left(\bar{T}_{b 6}\right)_{\{i k\}} \bar{B}^{j} \bar{D}^{k}\left(H_{8}\right)_{j}^{i}+j_{8} \Omega_{b b b}\left(\bar{T}_{b b}\right)_{\{i k\}} \bar{B}^{k} \bar{D}^{j}\left(H_{8}\right)_{j}^{i} .
\end{aligned}
$$

\begin{tabular}{|c|c|c|c|}
\hline Channel & Amplitude & Channel & Amplitude \\
\hline$\Omega_{b b b}^{-} \rightarrow \Xi_{b b}^{-} D^{0}$ & $j_{1} V_{c b} V_{u d}^{*}$ & $\Omega_{b b b}^{-} \rightarrow \Lambda_{b}^{0} B^{-} D^{0}$ & $-\left(j_{5}+j_{6}\right) V_{c b} V_{u c}^{*}$ \\
\hline$\Omega_{b b b}^{-} \rightarrow \Omega_{b b}^{-} D^{0}$ & $j_{1} V_{c b} V_{u s}^{*}$ & $\Omega_{b b b}^{-} \rightarrow \Xi_{b}^{0} B^{-} D^{0}$ & $-\left(j_{5}+j_{6}\right) V_{c b} V_{u s}^{*}$ \\
\hline$\Omega_{b b b}^{-} \rightarrow \Xi_{b b}^{0} D^{0} \pi^{-}$ & $\left(j_{2}+j_{4}\right) V_{c b} V_{u d}^{*}$ & $\Omega_{b b b}^{-} \rightarrow \Xi_{b}^{-} B^{-} D^{+}$ & $-j_{5} V_{c b} V_{u s}^{*}$ \\
\hline$\Omega_{b b b}^{-} \rightarrow \Xi_{b b}^{0} D^{0} K^{-}$ & $\left(j_{2}+j_{4}\right) V_{c b} V_{u s}^{*}$ & $\Omega_{b b b}^{-} \rightarrow \Xi_{b}^{-} B^{-} D_{s}^{+}$ & $j_{5} V_{c b} V_{u d}^{*}$ \\
\hline$\Omega_{b b b}^{-} \rightarrow \Xi_{b b}^{-} D^{0} \pi^{0}$ & $\frac{\left(j_{3}-j_{4}\right) V_{c b} V_{u d}^{*}}{\sqrt{2}}$ & $\Omega_{b b b}^{-} \rightarrow \Xi_{b}^{-} \bar{B}^{0} D^{0}$ & $-j_{6} V_{c b} V_{u s}^{*}$ \\
\hline$\Omega_{b b b}^{-} \rightarrow \Xi_{b b}^{-} D^{0} \bar{K}^{0}$ & $j_{4} V_{c b} V_{u s}^{*}$ & $\Omega_{b b b}^{-} \rightarrow \Xi_{b}^{-} \bar{B}_{s}^{0} D^{0}$ & $j_{6} V_{c b} V_{u d}^{*}$ \\
\hline$\Omega_{b b b}^{-} \rightarrow \Xi_{b b}^{-} D^{0} \eta$ & $\frac{\left(j_{3}+j_{4}\right) V_{c b} V_{u d}^{*}}{\sqrt{6}}$ & $\Omega_{b b b}^{-} \rightarrow \Sigma_{b}^{0} B^{-} D^{0}$ & $\frac{\left(j_{7}+j_{8}\right) V_{c b} V_{u d}^{*}}{\sqrt{2}}$ \\
\hline$\Omega_{b b b}^{-} \rightarrow \Xi_{b b}^{-} D^{+} \pi^{-}$ & $\left(j_{2}+j_{3}\right) V_{c b} V_{u d}^{*}$ & $\Omega_{b b b}^{-} \rightarrow \Sigma_{b}^{-} B^{-} D^{+}$ & $j_{7} V_{c b} V_{u d}^{*}$ \\
\hline$\Omega_{b b b}^{-} \rightarrow \Xi_{b b}^{-} D^{+} K^{-}$ & $j_{2} V_{c b} V_{u s}^{*}$ & $\Omega_{b b b}^{-} \rightarrow \Sigma_{b}^{-} \bar{B}^{0} D^{0}$ & $j_{8} V_{c b} V_{u d}^{*}$ \\
\hline$\Omega_{b b b}^{-} \rightarrow \Xi_{b b}^{-} D_{s}^{+} K^{-}$ & $j_{3} V_{c b} V_{u d}^{*}$ & $\Omega_{b b b}^{-} \rightarrow \Xi_{b}^{\prime 0} B^{-} D^{0}$ & $\frac{\left(j_{7}+j_{8}\right) V_{c b} V_{u s}^{*}}{\sqrt{2}}$ \\
\hline$\Omega_{b b b}^{-} \rightarrow \Omega_{b b}^{-} D^{0} \pi^{0}$ & $\frac{j_{3} V_{c b} V_{u s}^{*}}{\sqrt{2}}$ & $\Omega_{b b b}^{-} \rightarrow \Xi_{b}^{\prime-} B^{-} D^{+}$ & $\frac{j_{7} V_{c b} V_{u s}^{*}}{\sqrt{2}}$ \\
\hline$\Omega_{b b b}^{-} \rightarrow \Omega_{b b}^{-} D^{0} K^{0}$ & $j_{4} V_{c b} V_{u d}^{*}$ & $\Omega_{b b b}^{-} \rightarrow \Xi_{b}^{\prime-} B^{-} D_{s}^{+}$ & $\frac{j_{7} V_{c b} V_{u d}^{*}}{\sqrt{2}}$ \\
\hline$\Omega_{b b b}^{-} \rightarrow \Omega_{b b}^{-} D^{0} \eta$ & $\frac{\left(j_{3}-2 j_{4}\right) V_{c b} V_{u s}^{*}}{\sqrt{6}}$ & $\Omega_{b b b}^{-} \rightarrow \Xi_{b}^{\prime-} \bar{B}^{0} D^{0}$ & $\frac{j_{s} V_{c b} V_{u s}^{*}}{\sqrt{2}}$ \\
\hline$\Omega_{b b b}^{-} \rightarrow \Omega_{b b}^{-} D^{+} \pi^{-}$ & $j_{3} V_{c b} V_{u s}^{*}$ & $\Omega_{b b b}^{-} \rightarrow \Xi_{b}^{\prime-} \bar{B}_{s}^{0} D^{0}$ & $\frac{j_{8} V_{c b} V_{u d}^{*}}{\sqrt{2}}$ \\
\hline$\Omega_{b b b}^{-} \rightarrow \Omega_{b b}^{-} D_{s}^{+} \pi^{-}$ & $j_{2} V_{c b} V_{u d}^{*}$ & $\Omega_{b b b}^{-} \rightarrow \Omega_{b}^{-} B^{-} D_{s}^{+}$ & $j_{7} V_{c b} V_{u s}^{*}$ \\
\hline$\Omega_{b b b}^{-} \rightarrow \Omega_{b b}^{-} D_{s}^{+} K^{-}$ & $\left(j_{2}+j_{3}\right) V_{c b} V_{u s}^{*}$ & $\Omega_{b b b}^{-} \rightarrow \Omega_{b}^{-} \bar{B}_{s}^{0} D^{0}$ & $j_{8} V_{c b} V_{u s}^{*}$ \\
\hline
\end{tabular}

Decay amplitudes for different channels are obtained by expanding the above Hamiltonian and are collected in Table XIII.

TABLE XIII. Amplitudes for nonleptonic $\Omega_{b b b}^{-}$decays into a charmed meson plus a doubly bottom baryon or two bottom hadrons. 


\section{CKM-suppressed $\boldsymbol{b} \rightarrow \boldsymbol{u} \bar{c} d / s$ transition}

For the anticharm production, the operator having the quark contents $(\bar{u} b)(\bar{q} c)$ is given by

$$
\mathcal{H}_{\text {e.w. }}=\frac{G_{F}}{\sqrt{2}} V_{u b} V_{c q}^{*}\left[C_{1} O_{1}^{\bar{u} c}+C_{2} O_{2}^{\bar{u} c}\right]+\text { H.c. },
$$

with

$$
\begin{aligned}
& O_{1}^{\bar{u} c}=\bar{q}^{i} \gamma^{\mu}\left(1-\gamma_{5}\right) c^{j} \bar{u}^{j} \gamma_{\mu}\left(1-\gamma_{5}\right) b^{i}, \\
& O_{2}^{\bar{u} c}=\bar{q} \gamma^{\mu}\left(1-\gamma_{5}\right) c \bar{u} \gamma_{\mu}\left(1-\gamma_{5}\right) b .
\end{aligned}
$$

The two light antiquarks form the $\overline{\mathbf{3}}$ and $\mathbf{6}$ representations. The antisymmetric tensor $H_{3}^{\prime \prime}$ and the symmetric tensor $H_{6}$ have nonzero components

$$
\begin{aligned}
& \left(H_{\overline{3}}^{\prime \prime}\right)^{13}=-\left(H_{\overline{3}}^{\prime \prime}\right)^{31}=V_{u b} V_{c s}^{*}, \\
& \left(H_{6}\right)^{13}=\left(H_{6}\right)^{31}=V_{u b} V_{c s}^{*},
\end{aligned}
$$

for the $b \rightarrow u \bar{c} s$ transition. For the transition $b \rightarrow u \bar{c} d$, one requests the interchange of $2 \leftrightarrow 3$ in the subscripts, and $V_{c s}$ is replaced by $V_{c d}$.

The effective Hamiltonian is derived as

$$
\begin{aligned}
\mathcal{H}_{\mathrm{eff}}= & k_{1} \Omega_{b b b}\left(\bar{T}_{b b}\right)_{i} D_{j}\left(H_{\overline{3}}\right)^{i j}+k_{2} \Omega_{b b b}\left(\bar{T}_{b b}\right)_{i} D_{j}\left(H_{6}\right)^{i j}+k_{3} \Omega_{b b b}\left(\bar{T}_{b b}\right)_{i} D_{j}\left(M_{8}\right)_{k}^{i}\left(H_{\overline{3}}\right)^{j k}+k_{4} \Omega_{b b b}\left(\bar{T}_{b b}\right)_{i} D_{j}\left(M_{8}\right)_{k}^{j}\left(H_{\overline{3}}\right)^{i k} \\
& +k_{5} \Omega_{b b b}\left(\bar{T}_{b b}\right)_{i} D_{j}\left(M_{8}\right)_{k}^{i}\left(H_{6}\right)^{j k}+k_{6} \Omega_{b b b}\left(\bar{T}_{b b}\right)_{i} D_{j}\left(M_{8}\right)_{k}^{j}\left(H_{6}\right)^{i k}+k_{7} \Omega_{b b b}\left(\bar{T}_{b \overline{3}}\right)_{[i j]} \bar{B}^{k} D_{k}\left(H_{\overline{3}}\right)^{i j} \\
& +k_{8} \Omega_{b b b}\left(\bar{T}_{b \overline{3}}\right)_{[i k]} \bar{B}^{k} D_{j}\left(H_{\overline{3}}\right)^{i j}+k_{9} \Omega_{b b b}\left(\bar{T}_{b \overline{3}}\right)_{[i k]} \bar{B}^{k} D_{j}\left(H_{6}\right)^{i j}+k_{10} \Omega_{b b b}\left(\bar{T}_{b 6}\right)_{\{i k\}} \bar{B}^{k} D_{j}\left(H_{\overline{3}}\right)^{i j} \\
& +k_{11} \Omega_{b b b}\left(\bar{T}_{b 6}\right)_{\{i j\}} \bar{B}^{k} D_{k}\left(H_{6}\right)^{i j}+k_{12} \Omega_{b b b}\left(\bar{T}_{b 6}\right)_{\{i k\}} \bar{B}^{k} D_{j}\left(H_{6}\right)^{i j} .
\end{aligned}
$$

Decay amplitudes for different channels are obtained by expanding the above Hamiltonian and are collected in Table XIV.

\section{Charmless $b \rightarrow q_{1} \bar{q}_{2} q_{3}$ decays}

\section{Decays into a doubly bottom baryon bbq and a light meson}

The charmless $b \rightarrow q(q=d, s)$ transition is controlled by the weak Hamiltonian $\mathcal{H}_{\text {eff }}$,

$$
\mathcal{H}_{\text {e.w. }}=\frac{G_{F}}{\sqrt{2}}\left\{V_{u b} V_{u q}^{*}\left[C_{1} O_{1}^{\bar{u} u}+C_{2} O_{2}^{\bar{u} u}\right]-V_{t b} V_{t q}^{*}\left[\sum_{i=3}^{10} C_{i} O_{i}\right]\right\}+\text { H.c. }
$$

where $O_{i}$ is a four-quark operator or a moment-type operator. The four-quark operators $O_{i}$ are given as follows:

$$
\begin{aligned}
O_{1}^{\bar{u} u} & =\left(\bar{q}^{i} u^{j}\right)_{V-A}\left(\bar{u}^{j} b^{i}\right)_{V-A}, \quad O_{2}^{\bar{u} u}=(\bar{q} u)_{V-A}(\bar{u} b)_{V-A}, \quad O_{3}=(\bar{q} b)_{V-A} \sum_{q^{\prime}}\left(\bar{q}^{\prime} q^{\prime}\right)_{V-A}, \quad O_{4}=\left(\bar{q}^{i} b^{j}\right)_{V-A} \sum_{q^{\prime}}\left(\bar{q}^{\prime j} q^{\prime i}\right)_{V-A}, \\
O_{5} & =(\bar{q} b)_{V-A} \sum_{q^{\prime}}\left(\bar{q}^{\prime} q^{\prime}\right)_{V+A}, \quad O_{6}=\left(\bar{q}^{i} b^{j}\right)_{V-A} \sum_{q^{\prime}}\left(\bar{q}^{\prime j} q^{\prime i}\right)_{V+A}, \quad O_{7}=\frac{3}{2}(\bar{q} b)_{V-A} \sum_{q^{\prime}} e_{q^{\prime}}\left(\bar{q}^{\prime} q^{\prime}\right)_{V+A}, \\
O_{8} & =\frac{3}{2}\left(\bar{q}^{i} b^{j}\right)_{V-A} \sum_{q^{\prime}} e_{q^{\prime}}\left(\bar{q}^{\prime j} q^{\prime i}\right)_{V+A}, \quad O_{9}=\frac{3}{2}(\bar{q} b)_{V-A} \sum_{q^{\prime}} e_{q^{\prime}}\left(\bar{q}^{\prime} q^{\prime}\right)_{V-A}, \quad O_{10}=\frac{3}{2}\left(\bar{q}^{i} b^{j}\right)_{V-A} \sum_{q^{\prime}} e_{q^{\prime}}\left(\bar{q}^{\prime j} q^{\prime i}\right)_{V-A} \cdot
\end{aligned}
$$

In the above, $q$ denotes a $d$ quark for the $b \rightarrow d$ transition or an $s$ quark for the $b \rightarrow s$ transition, while $q^{\prime}=u, d, s$. $V$ and $A$ denote the vector and axial-vector currents. In the SU(3) group, penguin operators behave as the $\mathbf{3}$ representation, while tree operators can be decomposed in terms of a vector $H_{3}$, a traceless tensor antisymmetric in the upper indices $H_{\overline{6}}$, and a traceless tensor symmetric in the upper indices $H_{15}$.

For the $\Delta S=0(b \rightarrow d)$ decays, the nonzero components of the effective Hamiltonian are

$$
\begin{aligned}
\left(H_{3}\right)^{2} & =1, \quad\left(H_{\overline{6}}\right)_{1}^{12}=-\left(H_{\overline{6}}\right)_{1}^{21}=\left(H_{\overline{6}}\right)_{3}^{23}=-\left(H_{\overline{6}}\right)_{3}^{32}=1, \\
2\left(H_{15}\right)_{1}^{12} & =2\left(H_{15}\right)_{1}^{21}=-3\left(H_{15}\right)_{2}^{22}=-6\left(H_{15}\right)_{3}^{23}=-6\left(H_{15}\right)_{3}^{32}=6,
\end{aligned}
$$

and all other remaining entries are zero. For the $\Delta S=1(b \rightarrow s)$ decays, the nonzero entries in the $H_{\mathbf{3}}, H_{\overline{\mathbf{6}}}$, and $H_{15}$ are obtained from Eq. (77) with the exchange $2 \leftrightarrow 3$. 
TABLE XIV. Amplitudes for the CKM-suppressed $\Omega_{b b b}$ decays into an anticharmed meson.

\begin{tabular}{|c|c|c|c|}
\hline Channel & Amplitude & Channel & Amplitude \\
\hline$\Omega_{b b b}^{-} \rightarrow \Xi_{b b}^{0} D^{-}$ & $\left(k_{1}+k_{2}\right) V_{u b} V_{c d}^{*}$ & $\Omega_{b b b}^{-} \rightarrow \Lambda_{b}^{0} B^{-} \bar{D}^{0}$ & $\left(2 k_{7}+k_{8}-k_{9}\right) V_{u b} V_{c d}^{*}$ \\
\hline$\Omega_{b b b}^{-} \rightarrow \Xi_{b b}^{0} D_{s}^{-}$ & $\left(k_{1}+k_{2}\right) V_{u b} V_{c s}^{*}$ & $\Omega_{b b b}^{-} \rightarrow \Lambda_{b}^{0} \bar{B}^{0} D^{-}$ & $\left(2 k_{7}+k_{8}+k_{9}\right) V_{u b} V_{c d}^{*}$ \\
\hline$\Omega_{b b b}^{-} \rightarrow \Xi_{b b}^{-} \bar{D}^{0}$ & $\left(k_{2}-k_{1}\right) V_{u b} V_{c d}^{*}$ & $\Omega_{b b b}^{-} \rightarrow \Lambda_{b}^{0} \bar{B}^{0} D_{s}^{-}$ & $\left(k_{8}+k_{9}\right) V_{u b} V_{c s}^{*}$ \\
\hline$\Omega_{b b b}^{-} \rightarrow \Omega_{b b}^{-} \bar{D}^{0}$ & $\left(k_{2}-k_{1}\right) V_{u b} V_{c s}^{*}$ & $\Omega_{b b b}^{-} \rightarrow \Lambda_{b}^{0} \bar{B}_{s}^{0} D_{s}^{-}$ & $2 k_{7} V_{u b} V_{c d}^{*}$ \\
\hline$\Omega_{b b b}^{-} \rightarrow \Xi_{b b}^{0} \bar{D}^{0} \pi^{-}$ & $\left(k_{3}+k_{4}+k_{5}+k_{6}\right) V_{u b} V_{c d}^{*}$ & $\Omega_{b b b}^{-} \rightarrow \Xi_{b}^{0} B^{-} \bar{D}^{0}$ & $\left(2 k_{7}+k_{8}-k_{9}\right) V_{u b} V_{c s}^{*}$ \\
\hline$\Omega_{b b b}^{-} \rightarrow \Xi_{b b}^{0} \bar{D}^{0} K^{-}$ & $\left(k_{3}+k_{4}+k_{5}+k_{6}\right) V_{u b} V_{c s}^{*}$ & $\Omega_{b b b}^{-} \rightarrow \Xi_{b}^{0} \bar{B}^{0} D^{-}$ & $2 k_{7} V_{u b} V_{c s}^{*}$ \\
\hline$\Omega_{b b b}^{-} \rightarrow \Xi_{b b}^{0} D^{-} \pi^{0}$ & $-\frac{\left(k_{3}+k_{4}-k_{5}+k_{6}\right) V_{u b} V_{c d}^{*}}{\sqrt{2}}$ & $\Omega_{b b b}^{-} \rightarrow \Xi_{b}^{0} \bar{B}_{s}^{0} D^{-}$ & $\left(k_{8}+k_{9}\right) V_{u b} V_{c d}^{*}$ \\
\hline$\Omega_{b b b}^{-} \rightarrow \Xi_{b b}^{0} D^{-} \bar{K}^{0}$ & $\left(k_{4}+k_{6}\right) V_{u b} V_{c s}^{*}$ & $\Omega_{b b b}^{-} \rightarrow \Xi_{b}^{0} \bar{B}_{s}^{0} D_{s}^{-}$ & $\left(2 k_{7}+k_{8}+k_{9}\right) V_{u b} V_{c s}^{*}$ \\
\hline$\Omega_{b b b}^{-} \rightarrow \Xi_{b b}^{0} D^{-} \eta$ & $\frac{\left(-k_{3}+k_{4}+k_{5}+k_{6}\right) V_{u b} V_{c d}^{*}}{\sqrt{6}}$ & $\Omega_{b b b}^{-} \rightarrow \Xi_{b}^{-} \bar{B}^{0} \bar{D}^{0}$ & $\left(k_{8}-k_{9}\right) V_{u b} V_{c s}^{*}$ \\
\hline$\Omega_{b b b}^{-} \rightarrow \Xi_{b b}^{0} D_{s}^{-} \pi^{0}$ & $\frac{\left(k_{5}-k_{3}\right) V_{u b} V_{c s}^{*}}{\sqrt{2}}$ & $\Omega_{b b b}^{-} \rightarrow \Xi_{b}^{-} \bar{B}_{s}^{0} \bar{D}^{0}$ & $\left(k_{9}-k_{8}\right) V_{u b} V_{c d}^{*}$ \\
\hline$\Omega_{b b b}^{-} \rightarrow \Xi_{b b}^{0} D_{s}^{-} K^{0}$ & $\left(k_{4}+k_{6}\right) V_{u b} V_{c d}^{*}$ & $\Omega_{b b b}^{-} \rightarrow \Sigma_{b}^{+} B^{-} D^{-}$ & $\left(k_{10}+k_{12}\right) V_{u b} V_{c d}^{*}$ \\
\hline$\Omega_{b b b}^{-} \rightarrow \Xi_{b b}^{0} D_{s}^{-} \eta$ & $-\frac{\left(k_{3}+2 k_{4}-k_{5}+2 k_{6}\right) V_{u b} V_{c s}^{*}}{\sqrt{6}}$ & $\Omega_{b b b}^{-} \rightarrow \Sigma_{b}^{+} B^{-} D_{s}^{-}$ & $\left(k_{10}+k_{12}\right) V_{u b} V_{c s}^{*}$ \\
\hline$\Omega_{b b b}^{-} \rightarrow \Xi_{b b}^{-} \bar{D}^{0} \pi^{0}$ & $-\frac{\left(k_{3}+k_{4}+k_{5}-k_{6}\right) V_{u b} V_{c d}^{*}}{\sqrt{2}}$ & $\Omega_{b b b}^{-} \rightarrow \Sigma_{b}^{0} B^{-} \bar{D}^{0}$ & $\frac{\left(-k_{10}+2 k_{11}+k_{12}\right) V_{u b} V_{c d}^{*}}{\sqrt{2}}$ \\
\hline$\Omega_{b b b}^{-} \rightarrow \Xi_{b b}^{-} \bar{D}^{0} \bar{K}^{0}$ & $\left(k_{3}+k_{5}\right) V_{u b} V_{c s}^{*}$ & $\Omega_{b b b}^{-} \rightarrow \Sigma_{b}^{0} \bar{B}^{0} D^{-}$ & $\frac{\left(k_{10}+2 k_{11}+k_{12}\right) V_{u b} V_{c d}^{*}}{\sqrt{2}}$ \\
\hline$\Omega_{b b b}^{-} \rightarrow \Xi_{b b}^{-} \bar{D}^{0} \eta$ & $\frac{\left(k_{3}-k_{4}+k_{5}+k_{6}\right) V_{u b} V_{c d}^{*}}{\sqrt{6}}$ & $\Omega_{b b b}^{-} \rightarrow \Sigma_{b}^{0} \bar{B}^{0} D_{s}^{-}$ & $\frac{\left(k_{10}+k_{12}\right) V_{u b} V_{c s}^{*}}{\sqrt{2}}$ \\
\hline$\Omega_{b b b}^{-} \rightarrow \Xi_{b b}^{-} D^{-} \pi^{+}$ & $\left(-k_{3}-k_{4}+k_{5}+k_{6}\right) V_{u b} V_{c d}^{*}$ & $\Omega_{b b b}^{-} \rightarrow \Sigma_{b}^{0} \bar{B}_{s}^{0} D_{s}^{-}$ & $\sqrt{2} k_{11} V_{u b} V_{c d}^{*}$ \\
\hline$\Omega_{b b b}^{-} \rightarrow \Xi_{b b}^{-} D_{s}^{-} \pi^{+}$ & $\left(k_{5}-k_{3}\right) V_{u b} V_{c s}^{*}$ & $\Omega_{b b b}^{-} \rightarrow \Sigma_{b}^{-} \bar{B}^{0} \bar{D}^{0}$ & $\left(k_{12}-k_{10}\right) V_{u b} V_{c d}^{*}$ \\
\hline$\Omega_{b b b}^{-} \rightarrow \Xi_{b b}^{-} D_{s}^{-} K^{+}$ & $\left(k_{6}-k_{4}\right) V_{u b} V_{c d}^{*}$ & $\Omega_{b b b}^{-} \rightarrow \Xi_{b}^{\prime 0} B^{-} \bar{D}^{0}$ & $\frac{\left(-k_{10}+2 k_{11}+k_{12}\right) V_{u b} V_{c s}^{*}}{\sqrt{2}}$ \\
\hline$\Omega_{b b b}^{-} \rightarrow \Omega_{b b}^{-} \bar{D}^{0} \pi^{0}$ & $\frac{\left(k_{6}-k_{4}\right) V_{u b} V_{c s}^{*}}{\sqrt{2}}$ & $\Omega_{b b b}^{-} \rightarrow \Xi_{b}^{\prime 0} \bar{B}^{0} D^{-}$ & $\sqrt{2} k_{11} V_{u b} V_{c s}^{*}$ \\
\hline$\Omega_{b b b}^{-} \rightarrow \Omega_{b b}^{-} \bar{D}^{0} K^{0}$ & $\left(k_{3}+k_{5}\right) V_{u b} V_{c d}^{*}$ & $\Omega_{b b b}^{-} \rightarrow \Xi_{b}^{\prime 0} \bar{B}_{s}^{0} D^{-}$ & $\frac{\left(k_{10}+k_{12}\right) V_{u b} V_{c d}^{*}}{\sqrt{2}}$ \\
\hline$\Omega_{b b b}^{-} \rightarrow \Omega_{b b}^{-} \bar{D}^{0} \eta$ & $-\frac{\left(2 k_{3}+k_{4}+2 k_{5}-k_{6}\right) V_{u b} V_{c s}^{*}}{\sqrt{6}}$ & $\Omega_{b b b}^{-} \rightarrow \Xi_{b}^{\prime 0} \bar{B}_{s}^{0} D_{s}^{-}$ & $\frac{\left(k_{10}+2 k_{11}+k_{12}\right) V_{u b} V_{c s}^{*}}{\sqrt{2}}$ \\
\hline$\Omega_{b b b}^{-} \rightarrow \Omega_{b b}^{-} D^{-} \pi^{+}$ & $\left(k_{6}-k_{4}\right) V_{u b} V_{c s}^{*}$ & $\Omega_{b b b}^{-} \rightarrow \Xi_{b}^{\prime-} \bar{B}^{0} \bar{D}^{0}$ & $\frac{\left(k_{12}-k_{10}\right) V_{u b} V_{c s}^{*}}{\sqrt{2}}$ \\
\hline$\Omega_{b b b}^{-} \rightarrow \Omega_{b b}^{-} D^{-} K^{+}$ & $\left(k_{5}-k_{3}\right) V_{u b} V_{c d}^{*}$ & $\Omega_{b b b}^{-} \rightarrow \Xi_{b}^{\prime-} \bar{B}_{s}^{0} \bar{D}^{0}$ & $\frac{\left(k_{12}-k_{10}\right) V_{u b} V_{c d}^{*}}{\sqrt{2}}$ \\
\hline$\Omega_{b b b}^{-} \rightarrow \Omega_{b b}^{-} D_{s}^{-} K^{+}$ & $\left(-k_{3}-k_{4}+k_{5}+k_{6}\right) V_{u b} V_{c s}^{*}$ & $\Omega_{b b b}^{-} \rightarrow \Omega_{b}^{-} \bar{B}_{s}^{0} \bar{D}^{0}$ & $\left(k_{12}-k_{10}\right) V_{u b} V_{c s}^{*}$ \\
\hline
\end{tabular}

If the final state contains one light meson, the effective Hamiltonian is given as

$$
\mathcal{H}_{\mathrm{eff}}=l_{1} \Omega_{b b b}\left(\bar{T}_{b b}\right)_{j}\left(M_{8}\right)_{i}^{j}\left(H_{3}\right)^{i}+l_{2} \Omega_{b b b}\left(\bar{T}_{b b}\right)_{i}\left(M_{8}\right)_{j}^{k}\left(H_{\overline{6}}\right)_{k}^{i j}+l_{3} \Omega_{b b b}\left(\bar{T}_{b b}\right)_{i}\left(M_{8}\right)_{j}^{k}\left(H_{15}\right)_{k}^{i j}
$$

Decay amplitudes for different channels are obtained by expanding the above Hamiltonian and are collected in Table XV. With one additional light meson, we have

$$
\begin{aligned}
\mathcal{H}_{\mathrm{eff}}= & l_{4} \Omega_{b b b}\left(\bar{T}_{b b}\right)_{i}\left(M_{8}\right)_{j}^{i}\left(M_{8}\right)_{k}^{j}\left(H_{3}\right)^{k}+l_{5} \Omega_{b b b}\left(\bar{T}_{b b}\right)_{i}\left(M_{8}\right)_{k}^{j}\left(M_{8}\right)_{j}^{k}\left(H_{3}\right)^{i}+l_{6} \Omega_{b b b}\left(\bar{T}_{b b}\right)_{i}\left(M_{8}\right)_{l}^{k}\left(M_{8}\right)_{j}^{l}\left(H_{\overline{6}}\right)_{k}^{i j} \\
& +l_{7} \Omega_{b b b}\left(\bar{T}_{b b}\right)_{i}\left(M_{8}\right)_{j}^{i}\left(M_{8}\right)_{k}^{l}\left(H_{6}\right)_{l}^{j k}+l_{8} \Omega_{b b b}\left(\bar{T}_{b b}\right)_{i}\left(M_{8}\right)_{l}^{k}\left(M_{8}\right)_{j}^{l}\left(H_{15}\right)_{k}^{i j}+l_{9} \Omega_{b b b}\left(\bar{T}_{b b}\right)_{i}\left(M_{8}\right)_{j}^{i}\left(M_{8}\right)_{k}^{l}\left(H_{15}\right)_{l}^{j k} .
\end{aligned}
$$

Decay amplitudes for different channels are obtained by expanding the above Hamiltonian and are collected in Table XVI. A few remarks are given in order: 
TABLE XV. Amplitudes for $\Omega_{b b b}$ decays into a doubly bottom baryon and a light meson. The $b \rightarrow d$ transitions are given in the left columns, and the $b \rightarrow s$ ones are shown in the right columns.

\begin{tabular}{lccc}
\hline \hline Channel & Amplitude & Channel & Amplitude \\
\hline$\Omega_{b b b}^{-} \rightarrow \Xi_{b b}^{0} \pi^{-}$ & $l_{1}+l_{2}+3 l_{3}$ & $\Omega_{b b b}^{-} \rightarrow \Xi_{b b}^{0} K^{-}$ & $l_{1}^{\prime}+l_{2}^{\prime}+3 l_{3}^{\prime}$ \\
$\Omega_{b b b}^{-} \rightarrow \Xi_{b b}^{-} \pi^{0}$ & $-\frac{l_{1}+l_{2}-5 l_{3}}{\sqrt{2}}$ & $\Omega_{b b b}^{-} \rightarrow \Xi_{b b}^{-} \bar{K}^{0}$ & $l_{1}^{\prime}-l_{2}^{\prime}-l_{3}^{\prime}$ \\
$\Omega_{b b b}^{-} \rightarrow \Xi_{b b}^{-} \eta$ & $\frac{l_{1}-3 l_{2}+3 l_{3}}{\sqrt{6}}$ & $\Omega_{b b b}^{-} \rightarrow \Omega_{b b}^{-} \pi^{0}$ & $-\sqrt{2}\left(l_{2}^{\prime}-2 l_{3}^{\prime}\right)$ \\
$\Omega_{b b b}^{-} \rightarrow \Omega_{b b}^{-} K^{0}$ & $l_{1}-l_{2}-l_{3}$ & $\Omega_{b b b}^{-} \rightarrow \Omega_{b b}^{-} \eta$ & $-\sqrt{\frac{2}{3}}\left(l_{1}^{\prime}-3 l_{3}^{\prime}\right)$ \\
\hline \hline
\end{tabular}

(i) In Tables XV and XVI, both the $b \rightarrow d$ and $b \rightarrow s$ channels are included. Since the CKM matrix elements are different, the SU(3) irreducible amplitudes for the $b \rightarrow s$ transition are primed.

(ii) Expanding Eq. (79), one can find the amplitudes $l_{6}$ and $l_{7}$ are not independent and they always appear in the product $l_{6}-l_{7}$. So, in the two tables, we did not show the $l_{7}$.

(iii) Inspired from the $B$-meson decay data, we can infer that the typical branching fractions are at the order $10^{-6}$. Thus, these channels are rare decays and can be studied with a large amount of data. However, the direct $C P$ asymmetries in these channels are typically sizable.

(iv) For the $b \rightarrow q_{1} \bar{q}_{2} q_{3}$ decays, there are two amplitudes with different CKM factors. One can consider the U-spin connected decays with the decay amplitudes for $\bar{B}$ decays,

$$
\begin{aligned}
& A(\Delta S=0, \bar{B})=r\left(V_{u b} V_{u d}^{*} A^{T}+V_{t b} V_{t d}^{*} A^{P}\right), \\
& A(\Delta S=1, \bar{B})=V_{u b} V_{u s}^{*} A^{T}+V_{t b} V_{t s}^{*} A^{P},
\end{aligned}
$$

where $r$ is a constant factor and $A^{T}$ and $A^{P}$ are the amplitudes without the CKM factors. For the $B$ meson decays, we have the amplitudes

$$
\begin{aligned}
& A(\Delta S=0, B)=r\left(V_{u b}^{*} V_{u d} A^{T}+V_{t b}^{*} V_{t d} A^{P}\right), \\
& A(\Delta S=1, B)=V_{u b}^{*} V_{u s} A^{T}+V_{t b}^{*} V_{t s} A^{P},
\end{aligned}
$$

where the weak phases have been reversed. The $C P$-violating quantity $\Delta=\Gamma(\bar{B})-\Gamma(B)$ is then derived as

$$
\begin{aligned}
\Delta(\Delta S=0)= & 2 r^{2}\left(\operatorname{Re}\left[V_{u b} V_{u d}^{*} V_{t b}^{*} V_{t d} A^{T} A^{P *}\right]\right. \\
& \left.-\operatorname{Re}\left[V_{u b}^{*} V_{u d} V_{t b} V_{t d}^{*} A^{T} A^{P *}\right]\right) \\
= & -4 r^{2} \operatorname{Im}\left[V_{u b} V_{u d}^{*} V_{t b}^{*} V_{t d}\right] \operatorname{Im}\left[A^{T} A^{P *}\right],
\end{aligned}
$$

$$
\begin{aligned}
\Delta(\Delta S=1)= & 2\left(\operatorname{Re}\left[V_{u b} V_{u s}^{*} V_{t b}^{*} V_{t s} A^{T} A^{P *}\right]\right. \\
& \left.-\operatorname{Re}\left[V_{u b}^{*} V_{u s} V_{t b} V_{t s}^{*} A^{T} A^{P *}\right]\right) \\
= & -4 \operatorname{Im}\left[V_{u b} V_{u s}^{*} V_{t b}^{*} V_{t s}\right] \operatorname{Im}\left[A^{T} A^{P *}\right] .
\end{aligned}
$$

\begin{tabular}{|c|c|c|c|}
\hline Channel & Amplitude & Channel & Amplitude \\
\hline$\overline{\Omega_{b b b}^{-} \rightarrow \Xi_{b b}^{0} \pi^{-} \pi^{0}}$ & $4 \sqrt{2} l_{9}$ & $\Omega_{b b b}^{-} \rightarrow \Xi_{b b}^{0} \bar{K}^{0} \pi^{-}$ & $l_{4}^{\prime}+l_{6}^{\prime}+3 l_{8}^{\prime}-l_{9}^{\prime}$ \\
\hline$\Omega_{b b b}^{-} \rightarrow \Xi_{b b}^{0} K^{-} K^{0}$ & $l_{4}+l_{6}+3 l_{8}-l_{9}$ & $\Omega_{b b b}^{-} \rightarrow \Xi_{b b}^{0} K^{-} \pi^{0}$ & $\frac{l_{4}^{\prime}+l_{6}^{\prime}+3 l_{8}^{\prime}+7 l_{9}^{\prime}}{\sqrt{2}}$ \\
\hline$\Omega_{b b b}^{-} \rightarrow \Xi_{b b}^{0} \eta \pi^{-}$ & $\sqrt{\frac{2}{3}}\left(l_{4}+l_{6}+3 l_{8}+3 l_{9}\right)$ & $\Omega_{b b b}^{-} \rightarrow \Xi_{b b}^{0} K^{-} \eta$ & $-\frac{l_{4}^{\prime}+l_{6}^{\prime}+3 l_{l}^{\prime}-9 l_{9}^{\prime}}{\sqrt{6}}$ \\
\hline$\Omega_{b b b}^{-} \rightarrow \Xi_{b b}^{-} \pi^{0} \pi^{0}$ & $l_{4}+2 l_{5}-l_{6}+l_{8}-5 l_{9}$ & $\Omega_{b b b}^{-} \rightarrow \Xi_{b b}^{-} \pi^{+} K^{-}$ & $l_{4}^{\prime}-l_{6}^{\prime}-l_{8}^{\prime}+3 l_{9}^{\prime}$ \\
\hline$\Omega_{b b b}^{-} \rightarrow \Xi_{b b}^{-} \pi^{-} \pi^{+}$ & $l_{4}+2 l_{5}-l_{6}+l_{8}+3 l_{9}$ & $\Omega_{b b b}^{-} \rightarrow \Xi_{b b}^{-} \bar{K}^{0} \pi^{0}$ & $\frac{-l_{4}^{\prime \prime}+l_{6}^{\prime}+l_{8}^{\prime}+5 l_{9}^{\prime}}{\sqrt{2}}$ \\
\hline$\Omega_{b b b}^{-} \rightarrow \Xi_{b b}^{-} \bar{K}^{0} K^{0}$ & $l_{4}+2 l_{5}+l_{6}-3 l_{8}-l_{9}$ & $\Omega_{b b b}^{-} \rightarrow \Xi_{b b}^{-} \bar{K}^{0} \eta$ & $\frac{-l_{4}^{\prime}+l_{6}^{\prime}+l_{8}^{\prime}+5 l_{9}^{\prime}}{\sqrt{6}}$ \\
\hline$\Omega_{b b b}^{-} \rightarrow \Xi_{b b}^{-} K^{-} K^{+}$ & $2\left(l_{5}+l_{8}\right)$ & $\Omega_{b b b}^{-} \rightarrow \Omega_{b b}^{-} \pi^{+} \pi^{-}$ & $2\left(l_{5}^{\prime}+l_{8}^{\prime}\right)$ \\
\hline$\Omega_{b b b}^{-} \rightarrow \Xi_{b b}^{-} \eta \pi^{0}$ & $\frac{-l_{4}-l_{6}+5 l_{8}+l_{9}}{\sqrt{3}}$ & $\Omega_{b b b}^{-} \rightarrow \Omega_{b b}^{-} \pi^{0} \pi^{0}$ & $2 l_{5}^{\prime}+2 l_{8}^{\prime}$ \\
\hline$\Omega_{b b b}^{-} \rightarrow \Xi_{b b}^{-} \eta \eta$ & $\frac{1}{3}\left(l_{4}+3\left(2 l_{5}+l_{6}-l_{8}+l_{9}\right)\right)$ & $\Omega_{b b b}^{-} \rightarrow \Omega_{b b}^{-} \pi^{0} \eta$ & $-\frac{2\left(l_{6}^{\prime}-2 l_{8}^{\prime}+2 l_{9}^{\prime}\right)}{\sqrt{3}}$ \\
\hline$\Omega_{b b b}^{-} \rightarrow \Omega_{b b}^{-} \pi^{0} K^{0}$ & $\frac{-l_{4}+l_{6}+l_{8}+5 l_{9}}{\sqrt{2}}$ & $\Omega_{b b b}^{-} \rightarrow \Omega_{b b}^{-} K^{+} K^{-}$ & $l_{4}^{\prime}+2 l_{5}^{\prime}-l_{6}^{\prime}+l_{8}^{\prime}+3 l_{9}^{\prime}$ \\
\hline$\Omega_{b b b}^{-} \rightarrow \Omega_{b b}^{-} K^{+} \pi^{-}$ & $l_{4}-l_{6}-l_{8}+3 l_{9}$ & $\Omega_{b b b}^{-} \rightarrow \Omega_{b b}^{-} \bar{K}^{0} K^{0}$ & $l_{4}^{\prime}+2 l_{5}^{\prime}+l_{6}^{\prime}-3 l_{8}^{\prime}-l_{9}^{\prime}$ \\
\hline$\Omega_{b b b}^{-} \rightarrow \Omega_{b b}^{-} \eta K^{0}$ & $\frac{-l_{4}+l_{6}+l_{8}+5 l_{9}}{\sqrt{6}}$ & $\Omega_{b b b}^{-} \rightarrow \Omega_{b b}^{-} \eta \eta$ & $2\left(\frac{2 l_{4}^{\prime}}{3}+l_{5}^{\prime}-l_{8}^{\prime}-2 l_{9}^{\prime}\right)$ \\
\hline
\end{tabular}

Because of the property of the CKM matrix element $\operatorname{Im}\left[V_{u b} V_{u d}^{*} V_{t b}^{*} V_{t d}\right]=-\operatorname{Im}\left[V_{u b} V_{u s}^{*} V_{t b}^{*} V_{t s}\right]$, one has

$$
\Delta(\Delta S=0)=-r^{2} \Delta(\Delta S=1) .
$$

TABLE XVI. Amplitudes for $\Omega_{b b b}$ decays into a doubly bottom baryon and two light mesons. The $b \rightarrow d$ transitions are given in the left columns, and the $b \rightarrow s$ ones are shown in the right columns. The amplitude $l_{7}$ is neglected since it correlates with $l_{6}$. 
TABLE XVII. Amplitudes for $\Omega_{b b b}$ decays into a bottom baryon (antitriplet).

\begin{tabular}{|c|c|c|c|}
\hline Channel & Amplitude & Channel & Amplitude \\
\hline$\Omega_{b b b}^{-} \rightarrow \Lambda_{b}^{0} B^{-}$ & $m_{1}+2 m_{2}$ & $\Omega_{b b b}^{-} \rightarrow \Xi_{b}^{0} B^{-}$ & $m_{1}^{\prime}+2 m_{2}^{\prime}$ \\
\hline$\Omega_{b b b}^{-} \rightarrow \Xi_{b}^{-} \bar{B}_{s}^{0}$ & $2 m_{2}-m_{1}$ & $\Omega_{b b b}^{-} \rightarrow \Xi_{b}^{-} \bar{B}^{0}$ & $m_{1}^{\prime}-2 m_{2}^{\prime}$ \\
\hline$\Omega_{b b b}^{-} \rightarrow \Lambda_{b}^{0} B^{-} \pi^{0}$ & $\frac{m_{3}-m_{4}+2 m_{5}-m_{6}-5 m_{8}+6 m_{9}}{\sqrt{2}}$ & $\Omega_{b b b}^{-} \rightarrow \Lambda_{b}^{0} B^{-} \bar{K}^{0}$ & $m_{4}^{\prime}-m_{6}^{\prime}-m_{7}^{\prime}+m_{8}^{\prime}-3 m_{9}^{\prime}$ \\
\hline$\Omega_{b b b}^{-} \rightarrow \Lambda_{b}^{0} B^{-} \eta$ & $\frac{m_{3}+m_{4}+2 m_{5}-3 m_{6}-2 m_{7}-3 m_{8}}{\sqrt{6}}$ & $\Omega_{b b b}^{-} \rightarrow \Lambda_{b}^{0} \bar{B}^{0} K^{-}$ & $-m_{4}^{\prime}-m_{6}^{\prime}-m_{7}^{\prime}+3 m_{8}^{\prime}-m_{9}^{\prime}$ \\
\hline$\Omega_{b b b}^{-} \rightarrow \Lambda_{b}^{0} \bar{B}^{0} \pi^{-}$ & $m_{3}-m_{4}+2 m_{5}-m_{6}+3 m_{8}-2 m_{9}$ & $\Omega_{b b b}^{-} \rightarrow \Xi_{b}^{0} B^{-} \pi^{0}$ & $\frac{m_{3}^{\prime}+2 m_{5}^{\prime}-2 m_{6}^{\prime}-m_{7}^{\prime}-4 m_{8}^{\prime}+3 m_{9}^{\prime}}{\sqrt{2}}$ \\
\hline$\Omega_{b b b}^{-} \rightarrow \Lambda_{b}^{0} \bar{B}_{s}^{0} K^{-}$ & $m_{3}+2 m_{5}+m_{7}-m_{9}$ & $\Omega_{b b b}^{-} \rightarrow \Xi_{b}^{0} B^{-} \eta$ & $\frac{m_{3}^{\prime}-2 m_{4}^{\prime}+2 m_{5}^{\prime}+m_{7}^{\prime}-6 m_{8}^{\prime}+9 m_{9}^{\prime}}{\sqrt{6}}$ \\
\hline$\Omega_{b b b}^{-} \rightarrow \Xi_{b}^{0} B^{-} K^{0}$ & $m_{4}-m_{6}-m_{7}+m_{8}-3 m_{9}$ & $\Omega_{b b b}^{-} \rightarrow \Xi_{b}^{0} \bar{B}^{0} \pi^{-}$ & $m_{3}^{\prime}+2 m_{5}^{\prime}+m_{7}^{\prime}-m_{9}^{\prime}$ \\
\hline$\Omega_{b b b}^{-} \rightarrow \Xi_{b}^{0} \bar{B}_{s}^{0} \pi^{-}$ & $-m_{4}-m_{6}-m_{7}+3 m_{8}-m_{9}$ & $\Omega_{b b b}^{-} \rightarrow \Xi_{b}^{0} \bar{B}_{s}^{0} K^{-}$ & $m_{3}^{\prime}-m_{4}^{\prime}+2 m_{5}^{\prime}-m_{6}^{\prime}+3 m_{8}^{\prime}-2 m_{9}^{\prime}$ \\
\hline$\Omega_{b b b}^{-} \rightarrow \Xi_{b}^{-} B^{-} K^{+}$ & $-m_{3}+2 m_{5}+m_{7}-3 m_{9}$ & $\Omega_{b b b}^{-} \rightarrow \Xi_{b}^{-} B^{-} \pi^{+}$ & $m_{3}^{\prime}-2 m_{5}^{\prime}-m_{7}^{\prime}+3 m_{9}^{\prime}$ \\
\hline$\Omega_{b b b}^{-} \rightarrow \Xi_{b}^{-} \bar{B}^{0} K^{0}$ & $-m_{3}+m_{4}+2 m_{5}-m_{6}+m_{8}+2 m_{9}$ & $\Omega_{b b b}^{-} \rightarrow \Xi_{b}^{-} \bar{B}^{0} \pi^{0}$ & $-\frac{m_{3}^{\prime}-2 m_{5}^{\prime}+2 m_{6}^{\prime}+m_{7}^{\prime}+4 m_{8}^{\prime}-m_{9}^{\prime}}{\sqrt{2}}$ \\
\hline$\Omega_{b b b}^{-} \rightarrow \Xi_{b}^{-} \bar{B}_{s}^{0} \pi^{0}$ & $\frac{m_{4}+m_{6}+m_{7}+5 m_{8}+m_{9}}{\sqrt{2}}$ & $\Omega_{b b b}^{-} \rightarrow \Xi_{b}^{-} \bar{B}^{0} \eta$ & $\frac{m_{3}^{\prime}-2 m_{4}^{\prime}-2 m_{5}^{\prime}-m_{7}^{\prime}-6 m_{8}^{\prime}-3 m_{9}^{\prime}}{\sqrt{6}}$ \\
\hline$\Omega_{b b b}^{-} \rightarrow \Xi_{b}^{-} \bar{B}_{s}^{0} \eta$ & $\frac{2 m_{3}-m_{4}-4 m_{5}+3 m_{6}+m_{7}+3 m_{8}-3 m_{9}}{\sqrt{6}}$ & $\Omega_{b b b}^{-} \rightarrow \Xi_{b}^{-} \bar{B}_{s}^{0} \bar{K}^{0}$ & $m_{3}^{\prime}-m_{4}^{\prime}-2 m_{5}^{\prime}+m_{6}^{\prime}-m_{8}^{\prime}-2 m_{9}^{\prime}$ \\
\hline
\end{tabular}

TABLE XVIII. Amplitudes for $\Omega_{b b b}$ decays into a bottom baryon (sextet).

\begin{tabular}{|c|c|c|c|}
\hline Channel & Amplitude & Channel & Amplitude \\
\hline$\Omega_{b b b}^{-} \rightarrow \Sigma_{b}^{0} B^{-}$ & $\frac{n_{1}+6 n_{2}}{\sqrt{2}}$ & $\Omega_{b b b}^{-} \rightarrow \Xi_{b}^{\prime 0} B^{-}$ & $\frac{n_{1}^{\prime}+6 n_{2}^{\prime}}{\sqrt{2}}$ \\
\hline$\Omega_{b b b}^{-} \rightarrow \Sigma_{b}^{-} \bar{B}^{0}$ & $n_{1}-2 n_{2}$ & $\Omega_{b b b}^{-} \rightarrow \Xi_{b}^{\prime-} \bar{B}^{0}$ & $\frac{n_{1}^{\prime}-2 n_{2}^{\prime}}{\sqrt{2}}$ \\
\hline$\Omega_{b b b}^{-} \rightarrow \Xi_{b}^{\prime-} \bar{B}_{s}^{0}$ & $\frac{n_{1}-2 n_{2}}{\sqrt{2}}$ & $\Omega_{b b b}^{-} \rightarrow \Omega_{b}^{-} \bar{B}_{s}^{0}$ & $n_{1}^{\prime}-2 n_{2}^{\prime}$ \\
\hline$\Omega_{b b b}^{-} \rightarrow \Sigma_{b}^{+} B^{-} \pi^{-}$ & $n_{4}+3 n_{6}+3 n_{7}+n_{8}+n_{9}$ & $\Omega_{b b b}^{-} \rightarrow \Sigma_{b}^{+} B^{-} K^{-}$ & $n_{4}^{\prime}+3 n_{6}^{\prime}+3 n_{7}^{\prime}+n_{8}^{\prime}+n_{9}^{\prime}$ \\
\hline$\Omega_{b b b}^{-} \rightarrow \Sigma_{b}^{0} B^{-} \pi^{0}$ & $\frac{1}{2}\left(n_{3}-n_{4}+6 n_{5}+5 n_{6}-n_{8}-2 n_{9}\right)$ & $\Omega_{b b b}^{-} \rightarrow \Sigma_{b}^{0} B^{-} \bar{K}^{0}$ & $\frac{n_{4}^{\prime}-n_{6}^{\prime}+3 n_{7}^{\prime}-n_{8}^{\prime}+n_{9}^{\prime}}{\sqrt{2}}$ \\
\hline$\Omega_{b b b}^{-} \rightarrow \Sigma_{b}^{0} B^{-} \eta$ & $\frac{n_{3}+n_{4}+6 n_{5}+3 n_{6}+6 n_{7}-3 n_{8}}{2 \sqrt{3}}$ & $\Omega_{b b b}^{-} \rightarrow \Sigma_{b}^{0} \bar{B}^{0} K^{-}$ & $\frac{n_{4}^{\prime}+3 n_{6}^{\prime}-n_{7}^{\prime}+n_{8}^{\prime}-n_{9}^{\prime}}{\sqrt{2}}$ \\
\hline$\Omega_{b b b}^{-} \rightarrow \Sigma_{b}^{0} \bar{B}^{0} \pi^{-}$ & $\frac{n_{3}+n_{4}+6 n_{5}+3 n_{6}-2 n_{7}+n_{8}}{\sqrt{2}}$ & $\Omega_{b b b}^{-} \rightarrow \Sigma_{b}^{-} \bar{B}^{0} \bar{K}^{0}$ & $n_{4}^{\prime}-n_{6}^{\prime}-n_{7}^{\prime}-n_{8}^{\prime}-n_{9}^{\prime}$ \\
\hline$\Omega_{b b b}^{-} \rightarrow \Sigma_{b}^{0} \bar{B}_{s}^{0} K^{-}$ & $\frac{n_{3}+6 n_{5}-n_{7}+n_{9}}{\sqrt{2}}$ & $\Omega_{b b b}^{-} \rightarrow \Xi_{b}^{\prime 0} B^{-} \pi^{0}$ & $\frac{1}{2}\left(n_{3}^{\prime}+6 n_{5}^{\prime}+4 n_{6}^{\prime}+3 n_{7}^{\prime}-2 n_{8}^{\prime}-n_{9}^{\prime}\right)$ \\
\hline$\Omega_{b b b}^{-} \rightarrow \Sigma_{b}^{-} B^{-} \pi^{+}$ & $n_{3}-2 n_{5}+3 n_{7}-n_{9}$ & $\Omega_{b b b}^{-} \rightarrow \Xi_{b}^{\prime 0} B^{-} \eta$ & $\frac{n_{3}^{\prime}-2 n_{4}^{\prime}+6 n_{5}^{\prime}+6 n_{6}^{\prime}-3 n_{7}^{\prime}-3 n_{9}^{\prime}}{2 \sqrt{3}}$ \\
\hline$\Omega_{b b b}^{-} \rightarrow \Sigma_{b}^{-} \bar{B}^{0} \pi^{0}$ & $-\frac{n_{3}+n_{4}-2 n_{5}-5 n_{6}-2 n_{7}+n_{8}}{\sqrt{2}}$ & $\Omega_{b b b}^{-} \rightarrow \Xi_{b}^{\prime 0} \bar{B}^{0} \pi^{-}$ & $\frac{n_{3}^{\prime}+6 n_{5}^{\prime}-n_{7}^{\prime}+n_{9}^{\prime}}{\sqrt{2}}$ \\
\hline$\Omega_{b b b}^{-} \rightarrow \Sigma_{b}^{-} \bar{B}^{0} \eta$ & $\frac{n_{3}+n_{4}-2 n_{5}+3 n_{6}-2 n_{7}-3 n_{8}}{\sqrt{6}}$ & $\Omega_{b b b}^{-} \rightarrow \Xi_{b}^{\prime 0} \bar{B}_{s}^{0} K^{-}$ & $\frac{n_{3}^{\prime}+n_{4}^{\prime}+6 n_{5}^{\prime}+3 n_{6}^{\prime}-2 n_{7}^{\prime}+n_{8}^{\prime}}{\sqrt{2}}$ \\
\hline$\Omega_{b b b}^{-} \rightarrow \Sigma_{b}^{-} \bar{B}_{s}^{0} \bar{K}^{0}$ & $n_{3}-2 n_{5}-n_{7}+n_{9}$ & $\Omega_{b b b}^{-} \rightarrow \Xi_{b}^{\prime-} B^{-} \pi^{+}$ & $\frac{n_{3}^{\prime}-2 n_{5}^{\prime}+3 n_{7}^{\prime}-n_{9}^{\prime}}{\sqrt{2}}$ \\
\hline$\Omega_{b b b}^{-} \rightarrow \Xi_{b}^{\prime 0} B^{-} K^{0}$ & $\frac{n_{4}-n_{6}+3 n_{7}-n_{8}+n_{9}}{\sqrt{2}}$ & $\Omega_{b b b}^{-} \rightarrow \Xi_{b}^{\prime-} \bar{B}^{0} \pi^{0}$ & $\frac{1}{2}\left(-n_{3}^{\prime}+2 n_{5}^{\prime}+4 n_{6}^{\prime}+n_{7}^{\prime}-2 n_{8}^{\prime}-n_{9}^{\prime}\right)$ \\
\hline$\Omega_{b b b}^{-} \rightarrow \Xi_{b}^{\prime 0} \bar{B}_{s}^{0} \pi^{-}$ & $\frac{n_{4}+3 n_{6}-n_{7}+n_{8}-n_{9}}{\sqrt{2}}$ & $\Omega_{b b b}^{-} \rightarrow \Xi_{b}^{\prime-} \bar{B}^{0} \eta$ & $\frac{n_{3}^{\prime}-2 n_{4}^{\prime}-2 n_{5}^{\prime}+6 n_{6}^{\prime}+n_{7}^{\prime}+3 n_{9}^{\prime}}{2 \sqrt{3}}$ \\
\hline$\Omega_{b b b}^{-} \rightarrow \Xi_{b}^{\prime-} B^{-} K^{+}$ & $\frac{n_{3}-2 n_{5}+3 n_{7}-n_{9}}{\sqrt{2}}$ & $\Omega_{b b b}^{-} \rightarrow \Xi_{b}^{\prime-} \bar{B}_{s}^{0} \bar{K}^{0}$ & $\frac{n_{3}^{\prime}+n_{4}^{\prime}-2 n_{5}^{\prime}-n_{6}^{\prime}-2 n_{7}^{\prime}-n_{8}^{\prime}}{\sqrt{2}}$ \\
\hline$\Omega_{b b b}^{-} \rightarrow \Xi_{b}^{\prime-} \bar{B}^{0} K^{0}$ & $\frac{n_{3}+n_{4}-2 n_{5}-n_{6}-2 n_{7}-n_{8}}{\sqrt{2}}$ & $\Omega_{b b b}^{-} \rightarrow \Omega_{b}^{-} B^{-} K^{+}$ & $n_{3}^{\prime}-2 n_{5}^{\prime}+3 n_{7}^{\prime}-n_{9}^{\prime}$ \\
\hline$\Omega_{b b b}^{-} \rightarrow \Xi_{b}^{\prime-} \bar{B}_{s}^{0} \pi^{0}$ & $\frac{1}{2}\left(-n_{4}+5 n_{6}+n_{7}-n_{8}+n_{9}\right)$ & $\Omega_{b b b}^{-} \rightarrow \Omega_{b}^{-} \bar{B}^{0} K^{0}$ & $n_{3}^{\prime}-2 n_{5}^{\prime}-n_{7}^{\prime}+n_{9}^{\prime}$ \\
\hline$\Omega_{b b b}^{-} \rightarrow \Xi_{b}^{\prime-} \bar{B}_{s}^{0} \eta$ & $\frac{-2 n_{3}+n_{4}+4 n_{5}+3 n_{6}+n_{7}-3 n_{8}-3 n_{9}}{2 \sqrt{3}}$ & $\Omega_{b b b}^{-} \rightarrow \Omega_{b}^{-} \bar{B}_{s}^{0} \pi^{0}$ & $\sqrt{2}\left(2 n_{6}^{\prime}-n_{8}^{\prime}\right)$ \\
\hline$\Omega_{b b b}^{-} \rightarrow \Omega_{b}^{-} \bar{B}_{s}^{0} K^{0}$ & $n_{4}-n_{6}-n_{7}-n_{8}-n_{9}$ & $\Omega_{b b b}^{-} \rightarrow \Omega_{b}^{-} \bar{B}_{s}^{0} \eta$ & $-\sqrt{\frac{2}{3}}\left(n_{3}^{\prime}+n_{4}^{\prime}-2 n_{5}^{\prime}-3 n_{6}^{\prime}-2 n_{7}^{\prime}\right)$ \\
\hline
\end{tabular}




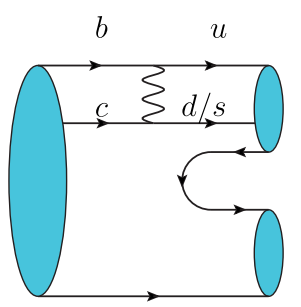

(a)

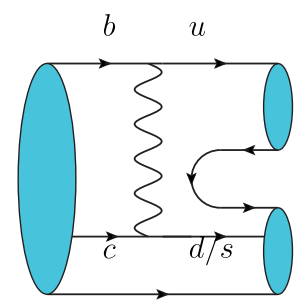

(b)
FIG. 5. Feynman diagrams for $\mathrm{W}$ exchange. The spectator is a bottom or a charm quark. If the final $u$ quark is replaced by a charm quark, the W-exchange contribution is an SU(3) triplet, and this triplet contribution is been incorporated in the $b \rightarrow q_{1} \bar{q}_{2} q_{3}$.
Since the $C P$-violating quantity is related to the $C P$ asymmetry $\Delta=A_{C P} \Gamma$, there exists a relation between the $C P$ asymmetry and decay widths $[29,32,60]$ :

$$
\frac{A_{C P}(\Delta S=0)}{A_{C P}(\Delta S=1)}=-r^{2} \frac{\Gamma(\Delta S=1)}{\Gamma(\Delta S=0)} .
$$

Such channel pairs include $\left[\Omega_{b b b}^{-} \rightarrow \Xi_{b b}^{0} \pi^{-}, \Omega_{b b b}^{-} \rightarrow\right.$ $\left.\Xi_{b b}^{0} K^{-}\right],\left[\Omega_{b b b}^{-} \rightarrow \Omega_{b b}^{-} K^{0}, \Omega_{b b b}^{-} \rightarrow \Omega_{b b}^{-} \bar{K}^{0}\right]$, etc. The future experimental data will be valuable to test flavor SU(3) symmetry and the CKM mechanism for $C P$ violation.

\section{Decays into a bottom meson and a bottom baryon bqq}

If the bottom baryon is an antitriplet, we have the effective Hamiltonian for two-body and three-body decays:

$$
\begin{aligned}
\mathcal{H}_{\mathrm{eff}}= & m_{1} \Omega_{b b b}\left(\bar{T}_{\mathbf{b} \overline{\mathbf{3}}}\right)_{[i j]} \bar{B}^{i}\left(H_{3}\right)^{j}+m_{2} \Omega_{b b b}\left(\bar{T}_{\mathbf{b} \overline{\mathbf{3}}}\right)_{[i j]} \bar{B}^{k}\left(H_{\overline{6}}\right)_{k}^{i j}+m_{3} \Omega_{b b b}\left(\bar{T}_{\mathbf{b} \overline{\mathbf{3}}}\right)_{[i j]} \bar{B}^{k}\left(M_{8}\right)_{k}^{i}\left(H_{3}\right)^{j} \\
& +m_{4} \Omega_{b b b}\left(\bar{T}_{\mathbf{b} \overline{\mathbf{3}}}\right)_{[i j]} \bar{B}^{i}\left(M_{8}\right)_{k}^{j}\left(H_{3}\right)^{k}+m_{5} \Omega_{b b b}\left(\bar{T}_{\mathbf{b} \overline{\mathbf{3}}}\right)_{[i j]} \bar{B}^{l}\left(M_{8}\right)_{l}^{k}\left(H_{\overline{6}}\right)_{k}^{i j}+m_{6} \Omega_{b b b}\left(\bar{T}_{\mathbf{b} \overline{\mathbf{3}}}\right)_{[i j]} \bar{B}^{i}\left(M_{8}\right)_{l}^{k}\left(H_{\overline{6}}\right)_{k}^{j l} \\
& +m_{7} \Omega_{b b b}\left(\bar{T}_{\mathbf{b} \overline{\mathbf{3}}}\right)_{[i j]} \bar{B}^{k}\left(M_{8}\right)_{l}^{i}\left(H_{\overline{6}}\right)_{k}^{j l}+m_{8} \Omega_{b b b}\left(\bar{T}_{\mathbf{b} \overline{\mathbf{3}}}\right)_{[i j]} \bar{B}^{j}\left(M_{8}\right)_{k}^{l}\left(H_{15}\right)_{l}^{i k}+m_{9} \Omega_{b b b}\left(\bar{T}_{\mathbf{b} \overline{\mathbf{3}}}\right)_{[i j]} \bar{B}^{l}\left(M_{8}\right)_{k}^{i}\left(H_{15}\right)_{l}^{j k} .
\end{aligned}
$$

Decay amplitudes for different channels are obtained by expanding the above Hamiltonian and are collected in Table XVII.

In the case of a sextet, we have

$$
\begin{aligned}
\mathcal{H}_{\mathrm{eff}}= & n_{1} \Omega_{b b b}\left(\bar{T}_{\mathbf{b 6}}\right)_{\{i j\}} \bar{B}^{i}\left(H_{3}\right)^{j}+n_{2} \Omega_{b b b}\left(\bar{T}_{\mathbf{b 6}}\right)_{\{i j\}} \bar{B}^{k}\left(H_{15}\right)_{k}^{i j}+n_{3} \Omega_{b b b}\left(\bar{T}_{\mathbf{b 6}}\right)_{\{i j\}} \bar{B}^{k}\left(M_{8}\right)_{k}^{i}\left(H_{3}\right)^{j} \\
& +n_{4} \Omega_{b b b}\left(\bar{T}_{\mathbf{b 6}}\right)_{\{i j\}} \bar{B}^{i}\left(M_{8}\right)_{k}^{j}\left(H_{3}\right)^{k}+n_{5} \Omega_{b b b}\left(\bar{T}_{\mathbf{b 6}}\right)_{\{i j\}} \bar{B}^{l}\left(M_{8}\right)_{l}^{k}\left(H_{15}\right)_{k}^{i j}+n_{6} \Omega_{b b b}\left(\bar{T}_{\mathbf{b 6}}\right)_{\{i j\}} \bar{B}^{i}\left(M_{8}\right)_{l}^{k}\left(H_{15}\right)_{k}^{j l} \\
& +n_{7} \Omega_{b b b}\left(\bar{T}_{\mathbf{b 6}}\right)_{\{i j\}} \bar{B}^{k}\left(M_{8}\right)_{l}^{i}\left(H_{15}\right)_{k}^{j l}+n_{8} \Omega_{b b b}\left(\bar{T}_{\mathbf{b 6}}\right)_{\{i j\}} \bar{B}^{j}\left(M_{8}\right)_{k}^{l}\left(H_{\overline{6}}\right)_{l}^{i k}+n_{9} \Omega_{b b b}\left(\bar{T}_{\mathbf{b 6}}\right)_{\{i j\}} \bar{B}^{l}\left(M_{8}\right)_{k}^{i}\left(H_{\overline{6}}\right)_{l}^{j k} .
\end{aligned}
$$

Decay amplitudes for different channels are obtained by expanding the above Hamiltonian and are collected in Table XVIII.

\section{NONLEPTONIC $\Omega_{c c b}$ AND $\Omega_{c b b}$ DECAYS}

For the mixed triply heavy baryons, $\Omega_{c c b}$ and $\Omega_{c b b}$, most of their weak decays can be obtained from the $\Omega_{c c c}$ and $\Omega_{b b b}$ decay channels with some replacements. For instance, decays of $\Omega_{c c b}$ induced by the charm quark can be obtained from the ones of $\Omega_{c c c}$ by replacing one charmed meson by the corresponding bottom meson, a charmed baryon by the corresponding bottom baryon, or a doubly charmed baryon $H_{c c}$ by its counterpart $H_{b c}$.

In addition, there are new decay modes, which are induced by W-exchange transition shown in Fig. $5,{ }^{1} b c \rightarrow u d$ or $b c \rightarrow u s$, with two heavy quarks annihilating into two light quarks. The spectator quark is a bottom or charm quark. These diagrams are dynamically suppressed by factors of $1 / m_{b, c}$. The electroweak Hamiltonian is similar to Eq. (73). Taking $\Omega_{c b b}$ as the example, one should notice that the final state contains only one heavy bottom quark. Thus, at the hadron level, the final state can be a bottom baryon (antitriplet) that has

$$
\begin{aligned}
\mathcal{H}_{\mathrm{eff}}= & a_{1} \Omega_{c b b}\left(\bar{T}_{b \overline{3}}\right)_{[i k]}\left(M_{8}\right)_{j}^{k}\left(H_{\overline{3}}^{\prime \prime}\right)^{[i j]}+a_{2} \Omega_{c b b}\left(\bar{T}_{b \overline{3}}\right)_{[i j]}\left(M_{8}\right)_{l}^{k}\left(M_{8}\right)_{k}^{l}\left(H_{\overline{3}}^{\prime \prime}\right)^{[i j]}+a_{3} \Omega_{c b b}\left(\bar{T}_{b \overline{3}}\right)_{[i k]}\left(M_{8}\right)_{l}^{k}\left(M_{8}\right)_{j}^{l}\left(H_{\overline{3}}^{\prime \prime}\right)^{[i j]} \\
& +a_{4} \Omega_{c b b}\left(\bar{T}_{b \overline{3}}\right)_{[k l]}\left(M_{8}\right)_{i}^{k}\left(M_{8}\right)_{j}^{l}\left(H_{\overline{3}}^{\prime \prime}\right)^{[i j]}+a_{5} \Omega_{c b b}\left(\bar{T}_{b \overline{3}}\right)_{[i k]}\left(M_{8}\right)_{j}^{k}\left(H_{6}^{\prime \prime}\right)^{\{i j\}}+a_{6} \Omega_{c b b}\left(\bar{T}_{b \overline{3}}\right)_{[i k]}\left(M_{8}\right)_{l}^{k}\left(M_{8}\right)_{j}^{l}\left(H_{6}^{\prime \prime}\right)^{\{i j\}} .
\end{aligned}
$$

Decay amplitudes for different channels are obtained by expanding the above Hamiltonian and are collected in Table XIX.

\footnotetext{
${ }^{1}$ If the final $u$ quark is replaced by a charm quark, the $\mathrm{W}$-exchange contribution is an $\mathrm{SU}(3)$ triplet, and this triplet contribution is incorporated in the $b \rightarrow q_{1} \bar{q}_{2} q_{3}$. In this case, the CKM matrix element is $V_{c b} V_{c d / c s}^{*}$.
} 
TABLE XIX. Amplitudes for $\Omega_{c b b}$ decays into a singly bottom baryon (triplet).

\begin{tabular}{|c|c|c|c|}
\hline Channel & Amplitude & Channel & Amplitude \\
\hline$\Omega_{c b b}^{0} \rightarrow \Lambda_{b}^{0} \pi^{0}$ & $-\sqrt{2} a_{5} V_{c d}^{*}$ & $\Omega_{c b b}^{0} \rightarrow \Xi_{b}^{0} K^{0}$ & $\left(a_{1}+a_{5}\right) V_{c d}^{*}$ \\
\hline$\Omega_{c b b}^{0} \rightarrow \Lambda_{b}^{0} \bar{K}^{0}$ & $\left(a_{1}+a_{5}\right) V_{c s}^{*}$ & $\Omega_{c b b}^{0} \rightarrow \Xi_{b}^{0} \eta$ & $-\frac{\left(a_{1}+3 a_{5}\right) V_{c s}^{*}}{\sqrt{6}}$ \\
\hline$\Omega_{c b b}^{0} \rightarrow \Lambda_{b}^{0} \eta$ & $\sqrt{\frac{2}{3}} a_{1} V_{c d}^{*}$ & $\Omega_{c b b}^{0} \rightarrow \Xi_{b}^{-} \pi^{+}$ & $\left(a_{1}-a_{5}\right) V_{c s}^{*}$ \\
\hline$\Omega_{c b b}^{0} \rightarrow \Xi_{b}^{0} \pi^{0}$ & $\frac{\left(a_{1}-a_{5}\right) V_{c s}^{*}}{\sqrt{2}}$ & $\Omega_{c b b}^{0} \rightarrow \Xi_{b}^{-} K^{+}$ & $\left(a_{5}-a_{1}\right) V_{c d}^{*}$ \\
\hline$\Omega_{c b b}^{0} \rightarrow \Lambda_{b}^{0} \pi^{0} \pi^{0}$ & $2\left(2 a_{2}+a_{3}-a_{4}\right) V_{c d}^{*}$ & $\Omega_{c b b}^{0} \rightarrow \Xi_{b}^{0} K^{+} \pi^{-}$ & $\left(a_{3}-2 a_{4}+a_{6}\right) V_{c d}^{*}$ \\
\hline$\Omega_{c b b}^{0} \rightarrow \Lambda_{b}^{0} \pi^{0} \bar{K}^{0}$ & $-\frac{\left(a_{3}-2 a_{4}+a_{6}\right) V_{c s}^{*}}{\sqrt{2}}$ & $\Omega_{c b b}^{0} \rightarrow \Xi_{b}^{0} K^{0} \pi^{0}$ & $-\frac{\left(a_{3}-2 a_{4}+a_{6}\right) V_{c d}^{*}}{\sqrt{2}}$ \\
\hline$\Omega_{c b b}^{0} \rightarrow \Lambda_{b}^{0} \pi^{-} \pi^{+}$ & $2\left(2 a_{2}+a_{3}-a_{4}\right) V_{c d}^{*}$ & $\Omega_{c b b}^{0} \rightarrow \Xi_{b}^{0} \bar{K}^{0} K^{0}$ & $\left(4 a_{2}+a_{3}+a_{6}\right) V_{c s}^{*}$ \\
\hline$\Omega_{c b b}^{0} \rightarrow \Lambda_{b}^{0} K^{+} K^{-}$ & $\left(4 a_{2}+a_{3}-a_{6}\right) V_{c d}^{*}$ & $\Omega_{c b b}^{0} \rightarrow \Xi_{b}^{0} K^{-} K^{+}$ & $2\left(2 a_{2}+a_{3}-a_{4}\right) V_{c s}^{*}$ \\
\hline$\Omega_{c b b}^{0} \rightarrow \Lambda_{b}^{0} K^{0} \bar{K}^{0}$ & $\left(4 a_{2}+a_{3}+a_{6}\right) V_{c d}^{*}$ & $\Omega_{c b b}^{0} \rightarrow \Xi_{b}^{0} \eta K^{0}$ & $-\frac{\left(a_{3}-2 a_{4}+a_{6}\right) V_{c d}^{*}}{\sqrt{6}}$ \\
\hline$\Omega_{c b b}^{0} \rightarrow \Lambda_{b}^{0} \bar{K}^{0} \eta$ & $-\frac{\left(a_{3}-2 a_{4}+a_{6}\right) V_{c s}^{*}}{\sqrt{6}}$ & $\Omega_{c b b}^{0} \rightarrow \Xi_{b}^{0} \eta \eta$ & $\frac{1}{3}\left(12 a_{2}+5 a_{3}-4 a_{4}+3 a_{6}\right) V_{c s}^{*}$ \\
\hline$\Omega_{c b b}^{0} \rightarrow \Lambda_{b}^{0} K^{-} \pi^{+}$ & $\left(a_{3}-2 a_{4}+a_{6}\right) V_{c s}^{*}$ & $\Omega_{c b b}^{0} \rightarrow \Xi_{b}^{-} \pi^{+} K^{0}$ & $\left(-a_{3}+2 a_{4}+a_{6}\right) V_{c d}^{*}$ \\
\hline$\Omega_{c b b}^{0} \rightarrow \Lambda_{b}^{0} \eta \pi^{0}$ & $-\frac{2 a_{6} V_{c d}^{*}}{\sqrt{3}}$ & $\Omega_{c b b}^{0} \rightarrow \Xi_{b}^{-} \pi^{+} \eta$ & $\sqrt{\frac{2}{3}}\left(a_{3}-2 a_{4}-a_{6}\right) V_{c s}^{*}$ \\
\hline$\Omega_{c b b}^{0} \rightarrow \Lambda_{b}^{0} \eta \eta$ & $\frac{2}{3}\left(6 a_{2}+a_{3}+a_{4}\right) V_{c d}^{*}$ & $\Omega_{c b b}^{0} \rightarrow \Xi_{b}^{-} K^{+} \pi^{0}$ & $\frac{\left(-a_{3}+2 a_{4}+a_{6}\right) V_{c d}^{*}}{\sqrt{2}}$ \\
\hline$\Omega_{c b b}^{0} \rightarrow \Xi_{b}^{0} \pi^{+} \pi^{-}$ & $\left(4 a_{2}+a_{3}-a_{6}\right) V_{c s}^{*}$ & $\Omega_{c b b}^{0} \rightarrow \Xi_{b}^{-} K^{+} \eta$ & $\frac{\left(a_{3}-2 a_{4}-a_{6}\right) V_{c d}^{*}}{\sqrt{6}}$ \\
\hline$\Omega_{c b b}^{0} \rightarrow \Xi_{b}^{0} \pi^{0} \pi^{0}$ & $\left(4 a_{2}+a_{3}-a_{6}\right) V_{c s}^{*}$ & $\Omega_{c b b}^{0} \rightarrow \Xi_{b}^{-} \bar{K}^{0} K^{+}$ & $\left(a_{3}-2 a_{4}-a_{6}\right) V_{c s}^{*}$ \\
\hline$\Omega_{c b b}^{0} \rightarrow \Xi_{b}^{0} \pi^{0} \eta$ & $\frac{\left(a_{3}-2 a_{4}-a_{6}\right) V_{c s}^{*}}{\sqrt{3}}$ & & \\
\hline
\end{tabular}

The final state can be a bottom baryon (sextet) that has

$$
\begin{aligned}
\mathcal{H}_{\mathrm{eff}}= & b_{1} \Omega_{c b b}\left(\bar{T}_{b 6}\right)_{\{i k\}}\left(M_{8}\right)_{j}^{k}\left(H_{\overline{3}}^{\prime \prime}\right)^{[i j]}+b_{2} \Omega_{c b b}\left(\bar{T}_{b 6}\right)_{\{i k\}}\left(M_{8}\right)_{l}^{k}\left(M_{8}\right)_{j}^{l}\left(H_{\overline{3}}^{\prime \prime}\right)^{[i j]}+b_{3} \Omega_{c b b}\left(\bar{T}_{b 6}\right)_{\{i k\}}\left(M_{8}\right)_{j}^{k}\left(H_{6}^{\prime \prime}\right)^{\{i j\}} \\
& +b_{5} \Omega_{c b b}\left(\bar{T}_{b 6}\right)_{\{i j\}}\left(M_{8}\right)_{l}^{k}\left(M_{8}\right)_{k}^{l}\left(H_{6}^{\prime \prime}\right)^{\{i j\}}+b_{6} \Omega_{c b b}\left(\bar{T}_{b 6}\right)_{\{i k\}}\left(M_{8}\right)_{l}^{k}\left(M_{8}\right)_{j}^{l}\left(H_{6}^{\prime \prime}\right)^{\{i j\}}+b_{7} \Omega_{c b b}\left(\bar{T}_{b 6}\right)_{\{k l\}}\left(M_{8}\right)_{i}^{k} M_{j}^{l}\left(H_{6}^{\prime \prime}\right)^{\{i j\}} .
\end{aligned}
$$

Decay amplitudes for different channels are obtained by expanding the above Hamiltonian and are collected in Table XX.

If the final state contains a bottom meson and a light baryon (octet), we have

$$
\begin{aligned}
\mathcal{H}_{\mathrm{eff}}= & c_{1} \Omega_{c b b} \bar{B}^{k} \epsilon_{i j l}\left(\bar{T}_{8}\right)_{k}^{l}\left(H_{\overline{3}}^{\prime \prime}\right)^{[i j]}+c_{2} \Omega_{c b b} \bar{B}^{i} \epsilon_{i j l}\left(\bar{T}_{8}\right)_{k}^{l}\left(H_{\overline{3}}^{\prime \prime}\right)^{[j k]}+c_{3} \Omega_{c b b} \bar{B}^{m} \epsilon_{i j l}\left(\bar{T}_{8}\right)_{k}^{l} M_{m}^{k}\left(H_{\overline{3}}^{\prime \prime}\right)^{[i j]} \\
& +c_{4} \Omega_{c b b} \bar{B}^{j} \epsilon_{i j l}\left(\bar{T}_{8}\right)_{k}^{l} M_{m}^{k}\left(H_{\overline{3}}^{\prime \prime}\right)^{[i m]}+c_{5} \Omega_{c b b} \bar{B}^{k} \epsilon_{i j l}\left(\bar{T}_{8}\right)_{k}^{l} M_{m}^{j}\left(H_{\overline{3}}^{\prime \prime}\right)^{[i m]}+c_{6} \Omega_{c b b} \bar{B}^{m} \epsilon_{i j l}\left(\bar{T}_{8}\right)_{k}^{l} M_{m}^{j}\left(H_{\overline{3}}^{\prime \prime}\right)^{[i k]} \\
& +c_{7} \Omega_{c b b} \bar{B}^{i} \epsilon_{i j l}\left(\bar{T}_{8}\right)_{k}^{l} M_{m}^{j}\left(H_{\overline{3}}^{\prime \prime}\right)^{[k m]}+c_{8} \Omega_{c b b} \bar{B}^{i} \epsilon_{i j l}\left(\bar{T}_{8}\right)_{k}^{l}\left(H_{6}^{\prime \prime}\right)^{\{j k\}}+c_{9} \Omega_{c b b} \bar{B}^{j} \epsilon_{i j l}\left(\bar{T}_{8}\right)_{k}^{l} M_{m}^{k}\left(H_{6}^{\prime \prime}\right)^{\{i m\}} \\
& +c_{10} \Omega_{c b b} \bar{B}^{k} \epsilon_{i j l}\left(\bar{T}_{8}\right)_{k}^{l} M_{m}^{j}\left(H_{6}^{\prime \prime}\right)^{\{i m\}}+c_{11} \Omega_{c b b} \bar{B}^{m} \epsilon_{i j l}\left(\bar{T}_{8}\right)_{k}^{l} M_{m}^{j}\left(H_{6}^{\prime \prime}\right)^{\{i k\}}+c_{12} \Omega_{c b b} \bar{B}^{i} \epsilon_{i j l}\left(\bar{T}_{8}\right)_{k}^{l} M_{m}^{j}\left(H_{6}^{\prime \prime}\right)^{\{k m\}} .
\end{aligned}
$$

Decay amplitudes for different channels are obtained by expanding the above Hamiltonian and are collected in Table XXI. One can find the amplitudes $c_{1}$ and $c_{2}$ are not independent; they always appear in the product $2 c_{1}-c_{2}$, So, in Table XXI, we do not show $c_{2}$.

If the final state contains a bottom meson and a light baryon (decuplet), we have

$$
\begin{aligned}
\mathcal{H}_{\mathrm{eff}}= & d_{1} \Omega_{c b b} \bar{B}^{j}\left(\bar{T}_{10}\right)_{i j k} M_{m}^{k}\left(H_{\overline{3}}^{\prime \prime}\right)^{[i m]}+d_{2} \Omega_{c b b} \bar{B}^{k}\left(\bar{T}_{10}\right)_{i j k} M_{m}^{j}\left(H_{\overline{3}}^{\prime \prime}\right)^{[i m]}+d_{3} \Omega_{c b b} \bar{B}^{i}\left(\bar{T}_{10}\right)_{i j k} M_{m}^{j}\left(H_{\overline{3}}^{\prime \prime}\right)^{[k m]} \\
& +d_{4} \Omega_{c b b} \bar{B}^{k}\left(\bar{T}_{10}\right)_{i j k}\left(H_{6}^{\prime \prime}\right)^{\{i j\}}+d_{5} \Omega_{c b b} \bar{B}^{i}\left(\bar{T}_{10}\right)_{i j k}\left(H_{6}^{\prime \prime}\right)^{\{j k\}}+d_{6} \Omega_{c b b} \bar{B}^{m}\left(\bar{T}_{10}\right)_{i j k} M_{m}^{k}\left(H_{6}^{\prime \prime}\right)^{\{i j\}} \\
& +d_{7} \Omega_{c b b} \bar{B}^{j}\left(\bar{T}_{10}\right)_{i j k} M_{m}^{k}\left(H_{6}^{\prime \prime}\right)^{\{i m\}}+d_{8} \Omega_{c b b} \bar{B}^{k}\left(\bar{T}_{10}\right)_{i j k} M_{m}^{j}\left(H_{6}^{\prime \prime}\right)^{\{i m\}} \\
& +d_{9} \Omega_{c b b} \bar{B}^{m}\left(\bar{T}_{10}\right)_{i j k} M_{m}^{j}\left(H_{6}^{\prime \prime}\right)^{\{i k\}}+d_{10} \Omega_{c b b} \bar{B}^{i}\left(\bar{T}_{10}\right)_{i j k} M_{m}^{j}\left(H_{6}^{\prime \prime}\right)^{\{k m\}} .
\end{aligned}
$$


TABLE XX. Amplitudes for $\Omega_{c b b}$ decays into a singly bottom baryon (sextet).

\begin{tabular}{|c|c|c|c|}
\hline Channel & Amplitude & Channel & Amplitude \\
\hline$\Omega_{c b b}^{0} \rightarrow \Sigma_{b}^{+} \pi^{-}$ & $\left(b_{1}+b_{4}\right) V_{c d}^{*}$ & $\Omega_{c b b}^{0} \rightarrow \Xi_{b}^{\prime 0} \pi^{0}$ & $\frac{1}{2}\left(b_{4}-b_{1}\right) V_{c s}^{*}$ \\
\hline$\Omega_{c b b}^{0} \rightarrow \Sigma_{b}^{+} K^{-}$ & $\left(b_{1}+b_{4}\right) V_{c s}^{*}$ & $\Omega_{c b b}^{0} \rightarrow \Xi_{b}^{\prime 0} K^{0}$ & $\frac{\left(b_{1}+b_{4}\right) V_{c d}^{*}}{\sqrt{2}}$ \\
\hline$\Omega_{c b b}^{0} \rightarrow \Sigma_{b}^{0} \pi^{0}$ & $-b_{1} V_{c d}^{*}$ & $\Omega_{c b b}^{0} \rightarrow \Xi_{b}^{\prime 0} \eta$ & $-\frac{\left(3 b_{1}+b_{4}\right) V_{c s}^{*}}{2 \sqrt{3}}$ \\
\hline$\Omega_{c b b}^{0} \rightarrow \Sigma_{b}^{0} \bar{K}^{0}$ & $\frac{\left(b_{1}+b_{4}\right) V_{c s}^{*}}{\sqrt{2}}$ & $\Omega_{c b b}^{0} \rightarrow \Xi_{b}^{\prime-} \pi^{+}$ & $\frac{\left(b_{4}-b_{1}\right) V_{c s}^{*}}{\sqrt{2}}$ \\
\hline$\Omega_{c b b}^{0} \rightarrow \Sigma_{b}^{0} \eta$ & $\frac{b_{4} V_{c d}^{*}}{\sqrt{3}}$ & $\Omega_{c b b}^{0} \rightarrow \Xi_{b}^{\prime-} K^{+}$ & $\frac{\left(b_{4}-b_{1}\right) V_{c d}^{*}}{\sqrt{2}}$ \\
\hline$\Omega_{c b b}^{0} \rightarrow \Sigma_{b}^{-} \pi^{+}$ & $\left(b_{4}-b_{1}\right) V_{c d}^{*}$ & $\Omega_{c b b}^{0} \rightarrow \Omega_{b}^{-} K^{+}$ & $\left(b_{4}-b_{1}\right) V_{c s}^{*}$ \\
\hline$\Omega_{c b b}^{0} \rightarrow \Sigma_{b}^{-} \pi^{+} \bar{K}^{0}$ & $2 b_{7} V_{c s}^{*}$ & $\Omega_{c b b}^{0} \rightarrow \Sigma_{b}^{-} \bar{K}^{0} K^{+}$ & $\left(b_{6}-b_{2}\right) V_{c d}^{*}$ \\
\hline$\Omega_{c b b}^{0} \rightarrow \Sigma_{b}^{+} \pi^{0} K^{-}$ & $\frac{\left(b_{2}+b_{6}+2 b_{7}\right) V_{c s}^{*}}{\sqrt{2}}$ & $\Omega_{c b b}^{0} \rightarrow \Sigma_{b}^{-} \eta \pi^{+}$ & $\sqrt{\frac{2}{3}}\left(-b_{2}+b_{6}+b_{7}\right) V_{c d}^{*}$ \\
\hline$\Omega_{c b b}^{0} \rightarrow \Sigma_{b}^{+} \pi^{-} \pi^{0}$ & $\sqrt{2} b_{7} V_{c d}^{*}$ & $\Omega_{c b b}^{0} \rightarrow \Xi_{b}^{\prime 0} \pi^{+} \pi^{-}$ & $\frac{\left(-b_{2}+4 b_{5}+b_{6}\right) V_{c s}^{*}}{\sqrt{2}}$ \\
\hline$\Omega_{c b b}^{0} \rightarrow \Sigma_{b}^{+} \pi^{-} \bar{K}^{0}$ & $\left(b_{2}+b_{6}\right) V_{c s}^{*}$ & $\Omega_{c b b}^{0} \rightarrow \Xi_{b}^{\prime 0} \pi^{0} \pi^{0}$ & $\frac{\left(-b_{2}+4 b_{5}+b_{6}\right) V_{c s}^{*}}{\sqrt{2}}$ \\
\hline$\Omega_{c b b}^{0} \rightarrow \Sigma_{b}^{+} \pi^{-} \eta$ & $\sqrt{\frac{2}{3}}\left(b_{2}+b_{6}+b_{7}\right) V_{c d}^{*}$ & $\Omega_{c b b}^{0} \rightarrow \Xi_{b}^{\prime 0} \pi^{0} K^{0}$ & $-\frac{1}{2}\left(b_{2}+b_{6}-2 b_{7}\right) V_{c d}^{*}$ \\
\hline$\Omega_{c b b}^{0} \rightarrow \Sigma_{b}^{+} K^{0} K^{-}$ & $\left(b_{2}+b_{6}\right) V_{c d}^{*}$ & $\Omega_{c b b}^{0} \rightarrow \Xi_{b}^{\prime 0} \pi^{0} \eta$ & $\frac{\left(-b_{2}+b_{6}-2 b_{7}\right) V_{c s}^{*}}{\sqrt{6}}$ \\
\hline$\Omega_{c b b}^{0} \rightarrow \Sigma_{b}^{0} \eta \eta$ & $\frac{2\left(6 b_{5}+b_{6}+b_{7}\right) V_{c d}^{*}}{3 \sqrt{2}}$ & $\Omega_{c b b}^{0} \rightarrow \Xi_{b}^{\prime 0} K^{+} \pi^{-}$ & $\frac{\left(b_{2}+b_{6}+2 b_{7}\right) V_{c d}^{*}}{\sqrt{2}}$ \\
\hline$\Omega_{c b b}^{0} \rightarrow \Sigma_{b}^{+} K^{-} \eta$ & $-\frac{\left(b_{2}+b_{6}-2 b_{7}\right) V_{c s}^{*}}{\sqrt{6}}$ & $\Omega_{c b b}^{0} \rightarrow \Xi_{b}^{\prime 0} K^{+} K^{-}$ & $\sqrt{2}\left(2 b_{5}+b_{6}+b_{7}\right) V_{c s}^{*}$ \\
\hline$\Omega_{c b b}^{0} \rightarrow \Omega_{b}^{-} K^{+} \eta$ & $\frac{\left(b_{2}-b_{6}-4 b_{7}\right) V_{c s}^{*}}{\sqrt{6}}$ & $\Omega_{c b b}^{0} \rightarrow \Xi_{b}^{\prime 0} K^{0} \bar{K}^{0}$ & $\frac{\left(b_{2}+4 b_{5}+b_{6}\right) V_{c s}^{*}}{\sqrt{2}}$ \\
\hline$\Omega_{c b b}^{0} \rightarrow \Sigma_{b}^{0} \pi^{+} \pi^{-}$ & $\sqrt{2}\left(2 b_{5}+b_{6}+b_{7}\right) V_{c d}^{*}$ & $\Omega_{c b b}^{0} \rightarrow \Xi_{b}^{\prime 0} K^{0} \eta$ & $-\frac{\left(b_{2}+b_{6}-2 b_{7}\right) V_{c d}^{*}}{2 \sqrt{3}}$ \\
\hline$\Omega_{c b b}^{0} \rightarrow \Sigma_{b}^{0} \pi^{+} K^{-}$ & $\frac{\left(b_{2}+b_{6}+2 b_{7}\right) V_{c s}^{*}}{\sqrt{2}}$ & $\Omega_{c b b}^{0} \rightarrow \Omega_{b}^{-} K^{+} \pi^{0}$ & $\frac{\left(b_{6}-b_{2}\right) V_{c s}^{*}}{\sqrt{2}}$ \\
\hline$\Omega_{c b b}^{0} \rightarrow \Sigma_{b}^{0} \pi^{0} \pi^{0}$ & $\frac{2\left(2 b_{5}+b_{6}-b_{7}\right) V_{c d}^{*}}{\sqrt{2}}$ & $\Omega_{c b b}^{0} \rightarrow \Omega_{b}^{-} \pi^{+} K^{0}$ & $\left(b_{6}-b_{2}\right) V_{c s}^{*}$ \\
\hline$\Omega_{c b b}^{0} \rightarrow \Sigma_{b}^{0} \pi^{0} \bar{K}^{0}$ & $-\frac{1}{2}\left(b_{2}+b_{6}-2 b_{7}\right) V_{c s}^{*}$ & $\Omega_{c b b}^{0} \rightarrow \Xi_{b}^{\prime-} K^{+} \eta$ & $\frac{\left(b_{2}-b_{6}+2 b_{7}\right) V_{c d}^{*}}{2 \sqrt{3}}$ \\
\hline$\Omega_{c b b}^{0} \rightarrow \Sigma_{b}^{0} \pi^{0} \eta$ & $-\sqrt{\frac{2}{3}} b_{2} V_{c d}^{*}$ & $\Omega_{c b b}^{0} \rightarrow \Xi_{b}^{\prime-} K^{+} \bar{K}^{0}$ & $\frac{\left(-b_{2}+b_{6}+2 b_{7}\right) V_{c s}^{*}}{\sqrt{2}}$ \\
\hline$\Omega_{c b b}^{0} \rightarrow \Omega_{b}^{-} K^{+} K^{0}$ & $2 b_{7} V_{c d}^{*}$ & $\Omega_{c b b}^{0} \rightarrow \Xi_{b}^{\prime 0} \eta \eta$ & $\frac{\left(3 b_{2}+12 b_{5}+5 b_{6}-4 b_{7}\right) V_{c s}^{*}}{3 \sqrt{2}}$ \\
\hline$\Omega_{c b b}^{0} \rightarrow \Sigma_{b}^{0} K^{+} K^{-}$ & $\frac{\left(-b_{2}+4 b_{5}+b_{6}\right) V_{c d}^{*}}{\sqrt{2}}$ & $\Omega_{c b b}^{0} \rightarrow \Xi_{b}^{\prime-} \pi^{+} K^{0}$ & $\frac{\left(-b_{2}+b_{6}+2 b_{7}\right) V_{c d}^{*}}{\sqrt{2}}$ \\
\hline$\Omega_{c b b}^{0} \rightarrow \Sigma_{b}^{0} K^{0} \bar{K}^{0}$ & $\frac{\left(b_{2}+4 b_{5}+b_{6}\right) V_{c d}^{*}}{\sqrt{2}}$ & $\Omega_{c b b}^{0} \rightarrow \Xi_{b}^{\prime-} \pi^{+} \eta$ & $\frac{\left(-b_{2}+b_{6}-2 b_{7}\right) V_{c s}^{*}}{\sqrt{3}}$ \\
\hline$\Omega_{c b b}^{0} \rightarrow \Sigma_{b}^{-} \pi^{+} \pi^{0}$ & $-\sqrt{2} b_{7} V_{c d}^{*}$ & $\Omega_{c b b}^{0} \rightarrow \Xi_{b}^{\prime-} K^{+} \pi^{0}$ & $-\frac{1}{2}\left(b_{2}-b_{6}+2 b_{7}\right) V_{c d}^{*}$ \\
\hline$\Omega_{c b b}^{0} \rightarrow \Sigma_{b}^{0} \bar{K}^{0} \eta$ & $-\frac{\left(b_{2}+b_{6}-2 b_{7}\right) V_{c s}^{*}}{2 \sqrt{3}}$ & & \\
\hline
\end{tabular}

Decay amplitudes for different channels are obtained by expanding the above Hamiltonian and are collected in Table XXII. It is interesting to notice that the above amplitudes $d_{i}$ are not all independent. The combinations of $d_{i} \mathrm{~s}, d_{1}+d_{2}+d_{3}, d_{4}+d_{5}, d_{6}+d_{9}$, and $d_{7}+d_{8}+d_{9}$ always appear in the amplitudes; thus, we have removed the $d_{2}, d_{3}, d_{5}, d_{9}, d_{8}$ and $d_{10}$ in Table XXII.

\section{GOLDEN CHANNELS}

Based on the above analysis, we first give a collection of the CKM-allowed decay channels for the $\Omega_{c c c}^{++}$in
Table XXIII and for the $\Omega_{b b b}$ in Table XXIV. The ones for $\Omega_{c c b}$ and $\Omega_{c b b}$ can be obtained by the replacements as discussed in the above section:

(i) The light pseudoscalar meson in these two tables can be replaced by its vector counterpart. For instance, a $\bar{K}^{0}$ can be replaced by a $\bar{K}^{* 0}$ decaying into $K^{-} \pi^{+}$.

(ii) Branching fractions for semileptonic $\Omega_{c c c}$ decay channels in Table XXIII can reach a few percent, but there is a neutrino in the final state, reducing somewhat the experimental efficiency.

(iii) Nonleptonic $\Omega_{c c c}$ such as $\Omega_{c c c}^{++} \rightarrow \Xi_{c c}^{++} K^{-} \pi^{+}$might be used to search for $\Omega_{c c c}$ especially at the LHC, 
TABLE XXI. Amplitudes for $\Omega_{c b b}$ decays into a bottom meson and a light baryon (octet).

\begin{tabular}{|c|c|c|c|}
\hline Channel & Amplitude & Channel & Amplitude \\
\hline$\Omega_{c b b}^{0} \rightarrow B^{-} \Sigma^{+}$ & $\left(-2 c_{1}-c_{8}\right) V_{u b} V_{c s}^{*}$ & $\Omega_{c b b}^{0} \rightarrow \bar{B}_{s}^{0} \Lambda^{0}$ & $\sqrt{\frac{2}{3}}\left(-2 c_{1}\right) V_{u b} V_{c d}^{*}$ \\
\hline$\Omega_{c b b}^{0} \rightarrow \bar{B}^{0} \Lambda^{0}$ & $\frac{\left(-2 c_{1}+3 c_{8}\right) V_{u b} V_{c s}^{*}}{\sqrt{6}}$ & $\Omega_{c b b}^{0} \rightarrow \bar{B}_{s}^{0} \Sigma^{0}$ & $-\sqrt{2} c_{8} V_{u b} V_{c d}^{*}$ \\
\hline$\Omega_{c b b}^{0} \rightarrow \bar{B}^{0} \Sigma^{0}$ & $\frac{\left(2 c_{1}+c_{8}\right) V_{u b} V_{c s}^{*}}{\sqrt{2}}$ & $\Omega_{c b b}^{0} \rightarrow \bar{B}_{s}^{0} \Xi^{0}$ & $\left(-2 c_{1}+c_{8}\right) V_{u b} V_{c s}^{*}$ \\
\hline$\Omega_{c b b}^{0} \rightarrow \bar{B}^{0} n$ & $\left(2 c_{1}-c_{8}\right) V_{u b} V_{c d}^{*}$ & $\Omega_{c b b}^{0} \rightarrow B^{-} p$ & $\left(2 c_{1}+c_{8}\right) V_{u b} V_{c d}^{*}$ \\
\hline$\Omega_{c b b}^{0} \rightarrow B^{-} \Lambda^{0} \pi^{+}$ & $-\frac{\left(2 c_{3}+c_{4}-c_{5}+c_{6}-2 c_{7}-c_{9}+c_{10}+3 c_{11}+2 c_{12}\right) V_{u b} V_{c s}^{*}}{\sqrt{6}}$ & $\Omega_{c b b}^{0} \rightarrow \bar{B}^{0} n \bar{K}^{0}$ & $\left(c_{4}+c_{5}+c_{9}+c_{10}\right) V_{u b} V_{c s}^{*}$ \\
\hline$\Omega_{c b b}^{0} \rightarrow B^{-} \Lambda^{0} K^{+}$ & $-\frac{\left(4 c_{3}+2 c_{4}+c_{5}+2 c_{6}-c_{7}-2 c_{9}-c_{10}+c_{12}\right) V_{u b} V_{c d}^{*}}{\sqrt{6}}$ & $\Omega_{c b b}^{0} \rightarrow \bar{B}^{0} n \eta$ & $\frac{\left(2 c_{3}+c_{4}+2 c_{5}+c_{6}+c_{7}+c_{9}+c_{11}-c_{12}\right) V_{u b} V_{c d}^{*}}{\sqrt{6}}$ \\
\hline$\Omega_{c b b}^{0} \rightarrow B^{-} \Sigma^{+} \pi^{0}$ & $\frac{\left(-2 c_{3}-c_{4}-c_{5}-c_{6}+c_{9}+c_{10}+c_{11}\right) V_{u b} V_{c s}^{*}}{\sqrt{2}}$ & $\Omega_{c b b}^{0} \rightarrow \bar{B}^{0} \Xi^{-} K^{+}$ & $\left(c_{4}-c_{7}-c_{9}+c_{12}\right) V_{u b} V_{c s}^{*}$ \\
\hline$\Omega_{c b b}^{0} \rightarrow B^{-} \Sigma^{+} K^{0}$ & $-\left(c_{5}+c_{7}+c_{10}+c_{12}\right) V_{u b} V_{c d}^{*}$ & $\Omega_{c b b}^{0} \rightarrow \bar{B}^{0} \Xi^{0} K^{0}$ & $-\left(2 c_{3}+c_{6}+c_{11}\right) V_{u b} V_{c s}^{*}$ \\
\hline$\Omega_{c b b}^{0} \rightarrow B^{-} \Sigma^{+} \eta$ & $\frac{\left(-2 c_{3}-c_{4}+c_{5}-c_{6}+2 c_{7}+c_{9}+3 c_{10}+c_{11}+2 c_{12}\right) V_{u b} V_{c s}^{*}}{\sqrt{6}}$ & $\Omega_{c b b}^{0} \rightarrow \bar{B}_{s}^{0} \Lambda^{0} \pi^{0}$ & $\frac{\left(c_{9}+2 c_{10}+c_{12}\right) V_{u b} V_{c d}^{*}}{\sqrt{3}}$ \\
\hline$\Omega_{c b b}^{0} \rightarrow B^{-} \Sigma^{0} \pi^{+}$ & $\frac{\left(2 c_{3}+c_{4}+c_{5}+c_{6}-c_{9}-c_{10}-c_{11}\right) V_{u b} V_{c s}^{*}}{\sqrt{2}}$ & $\Omega_{c b b}^{0} \rightarrow \bar{B}_{s}^{0} \Lambda^{0} \bar{K}^{0}$ & $-\frac{\left(2 c_{3}+c_{4}+2 c_{5}+c_{6}+c_{7}+c_{9}+2 c_{10}+3 c_{11}+c_{12}\right) V_{u b} V_{c 4}^{*}}{\sqrt{6}}$ \\
\hline$\Omega_{c b b}^{0} \rightarrow B^{-} \Sigma^{0} K^{+}$ & $\frac{\left(-c_{5}-c_{7}+c_{10}+2 c_{11}+c_{12}\right) V_{u b} V_{c d}^{*}}{\sqrt{2}}$ & $\Omega_{c b b}^{0} \rightarrow \bar{B}_{s}^{0} \Lambda^{0} \eta$ & $\frac{1}{3}\left(4 c_{3}-c_{4}-2 c_{5}+2 c_{6}-c_{7}\right) V_{u b} V_{c d}^{*}$ \\
\hline$\Omega_{c b b}^{0} \rightarrow B^{-} n \pi^{+}$ & $\left(2 c_{3}+c_{4}+c_{6}-c_{7}-c_{9}+c_{11}+c_{12}\right) V_{u b} V_{c d}^{*}$ & $\Omega_{c b b}^{0} \rightarrow \bar{B}_{s}^{0} \Sigma^{+} \pi^{-}$ & $\left(-c_{4}+c_{7}-c_{9}+c_{12}\right) V_{u b} V_{c d}^{*}$ \\
\hline$\Omega_{c b b}^{0} \rightarrow B^{-} \Xi^{0} K^{+}$ & $-\left(2 c_{3}+c_{4}+c_{6}-c_{7}-c_{9}+c_{11}+c_{12}\right) V_{u b} V_{c s}^{*}$ & $\Omega_{c b b}^{0} \rightarrow \bar{B}_{s}^{0} \Sigma^{+} K^{-}$ & $\begin{array}{l}\left(-2 c_{3}-c_{4}-c_{6}+c_{7}-c_{9}\right. \\
\left.\quad+c_{11}+c_{12}\right) V_{u b} V_{c s}^{*}\end{array}$ \\
\hline$\Omega_{c b b}^{0} \rightarrow \bar{B}^{0} \Lambda^{0} \pi^{0}$ & $\frac{\left(2 c_{3}+c_{4}-c_{5}+c_{6}-2 c_{7}-c_{9}+c_{10}+3 c_{11}+2 c_{12}\right) V_{u b} V_{c s}^{*}}{2 \sqrt{3}}$ & $\Omega_{c b b}^{0} \rightarrow \bar{B}_{s}^{0} \Sigma^{0} \pi^{0}$ & $\left(c_{7}-c_{4}\right) V_{u b} V_{c d}^{*}$ \\
\hline$\Omega_{c b b}^{0} \rightarrow \bar{B}^{0} \Lambda^{0} K^{0}$ & $-\frac{\left(4 c_{3}+2 c_{4}+c_{5}+2 c_{6}-c_{7}+2 c_{9}+c_{10}-c_{12}\right) V_{u b} V_{c d}^{*}}{\sqrt{6}}$ & $\Omega_{c b b}^{0} \rightarrow \bar{B}_{s}^{0} \Sigma^{0} \bar{K}^{0}$ & $\frac{\left(2 c_{3}+c_{4}+c_{6}-c_{7}+c_{9}-c_{11}-c_{12}\right) V_{u b} V_{c s}^{*}}{\sqrt{2}}$ \\
\hline$\Omega_{c b b}^{0} \rightarrow \bar{B}^{0} \Lambda^{0} \eta$ & $\begin{array}{l}\frac{1}{6}\left(-2 c_{3}+5 c_{4}+c_{5}-c_{6}-4 c_{7}\right. \\
\left.\quad+3 c_{9}+3 c_{10}-3 c_{11}\right) V_{u b} V_{c s}^{*}\end{array}$ & $\Omega_{c b b}^{0} \rightarrow \bar{B}_{s}^{0} \Sigma^{0} \eta$ & $\frac{\left(c_{9}-2 c_{11}-c_{12}\right) V_{u b} V_{c d}^{*}}{\sqrt{3}}$ \\
\hline$\Omega_{c b b}^{0} \rightarrow \bar{B}^{0} \Sigma^{+} \pi^{-}$ & $\left(-2 c_{3}-c_{6}+c_{11}\right) V_{u b} V_{c s}^{*}$ & $\Omega_{c b b}^{0} \rightarrow \bar{B}_{s}^{0} \Sigma^{-} \pi^{+}$ & $\left(-c_{4}+c_{7}+c_{9}-c_{12}\right) V_{u b} V_{c d}^{*}$ \\
\hline$\Omega_{c b b}^{0} \rightarrow \bar{B}^{0} \Sigma^{0} \pi^{0}$ & $-\frac{1}{2}\left(2 c_{3}-c_{4}-c_{5}+c_{6}+c_{9}+c_{10}-c_{11}\right) V_{u b} V_{c s}^{*}$ & $\Omega_{c b b}^{0} \rightarrow \bar{B}_{s}^{0} n \bar{K}^{0}$ & $\left(2 c_{3}+c_{6}+c_{11}\right) V_{u b} V_{c d}^{*}$ \\
\hline$\Omega_{c b b}^{0} \rightarrow \bar{B}^{0} \Sigma^{0} K^{0}$ & $\frac{\left(c_{5}+c_{7}+c_{10}+2 c_{11}+c_{12}\right) V_{u b} V_{c d}^{*}}{\sqrt{2}}$ & $\Omega_{c b b}^{0} \rightarrow \bar{B}_{s}^{0} \Xi^{-} \pi^{+}$ & $\left(c_{5}+c_{7}-c_{10}-c_{12}\right) V_{u b} V_{c s}^{*}$ \\
\hline$\Omega_{c b b}^{0} \rightarrow \bar{B}^{0} \Sigma^{0} \eta$ & $\frac{\left(2 c_{3}+c_{4}-c_{5}+c_{6}-2 c_{7}-c_{9}-3 c_{10}-c_{11}-2 c_{12}\right) V_{u b} V_{c s}^{*}}{2 \sqrt{3}}$ & $\Omega_{c b b}^{0} \rightarrow \bar{B}_{s}^{0} \Xi^{-} K^{+}$ & $\left(-c_{4}-c_{5}+c_{9}+c_{10}\right) V_{u b} V_{c d}^{*}$ \\
\hline$\Omega_{c b b}^{0} \rightarrow \bar{B}^{0} \Sigma^{-} \pi^{+}$ & $\left(c_{4}+c_{5}-c_{9}-c_{10}\right) V_{u b} V_{c s}^{*}$ & $\Omega_{c b b}^{0} \rightarrow \bar{B}_{s}^{0} \Xi^{0} \pi^{0}$ & $\frac{\left(-c_{5}-c_{7}+c_{10}+c_{12}\right) V_{u b} V_{c s}^{*}}{\sqrt{2}}$ \\
\hline$\Omega_{c b b}^{0} \rightarrow \bar{B}^{0} \Sigma^{-} K^{+}$ & $\left(-c_{5}-c_{7}+c_{10}+c_{12}\right) V_{u b} V_{c d}^{*}$ & $\Omega_{c b b}^{0} \rightarrow \bar{B}_{s}^{0} \Xi^{0} K^{0}$ & $-\left(c_{4}+c_{5}+c_{9}+c_{10}\right) V_{u b} V_{c d}^{*}$ \\
\hline$\Omega_{c b b}^{0} \rightarrow \bar{B}^{0} n \pi^{0}$ & $-\frac{\left(2 c_{3}+c_{4}+c_{6}-c_{7}+c_{9}+2 c_{10}+c_{11}+c_{12}\right) V_{u b} V_{c d}^{*}}{\sqrt{2}}$ & $\Omega_{c b b}^{0} \rightarrow \bar{B}_{s}^{0} \Xi^{0} \eta$ & $\frac{\left(4 c_{3}+2 c_{4}+c_{5}+2 c_{6}-c_{7}+2 c_{9}+3 c_{10}+2 c_{11}+c_{12}\right) V_{u b} V_{c s}^{*}}{\sqrt{6}}$ \\
\hline$\Omega_{c b b}^{0} \rightarrow B^{-} p \pi^{0}$ & $\frac{\left(2 c_{3}+c_{4}+c_{6}-c_{7}-c_{9}-2 c_{10}-c_{11}-c_{12}\right) V_{u b} V_{c d}^{*}}{\sqrt{2}}$ & $\Omega_{c b b}^{0} \rightarrow B^{-} p \bar{K}^{0}$ & $\left(c_{5}+c_{7}+c_{10}+c_{12}\right) V_{u b} V_{\mathrm{cs}}^{*}$ \\
\hline$\Omega_{c b b}^{0} \rightarrow B^{-} p \eta$ & $\frac{\left(2 c_{3}+c_{4}+2 c_{5}+c_{6}+c_{7}-c_{9}-c_{11}+c_{12}\right) V_{u b} V_{\mathrm{cd}}^{*}}{\sqrt{6}}$ & $\Omega_{c b b}^{0} \rightarrow \bar{B}^{0} p \pi^{-}$ & $\begin{array}{l}\left(2 c_{3}+c_{4}+c_{6}-c_{7}+c_{9}\right. \\
\left.-c_{11}-c_{12}\right) V_{u b} V_{\mathrm{cd}}^{*}\end{array}$ \\
\hline$\Omega_{c b b}^{0} \rightarrow \bar{B}^{0} p K^{-}$ & $\left(c_{4}-c_{7}+c_{9}-c_{12}\right) V_{u b} V_{\mathrm{cs}}^{*}$ & $\Omega_{c b b}^{0} \rightarrow \bar{B}_{s}^{0} p K^{-}$ & $\left(2 c_{3}+c_{6}-c_{11}\right) V_{u b} V_{\mathrm{cd}}^{*}$ \\
\hline
\end{tabular}

since their branching fractions are sizable, and the final state can be easy to identify. This will make use of the doubly heavy baryon $\Xi_{c c}^{++}$, which has just been discovered by LHCb.

(iv) For nonleptonic decays of $\Omega_{b b b}^{-}$, the largest branching fraction might reach $10^{-3}$. Taking into account its daughter decays, we expect that the branching fraction for $\Omega_{b b b}^{-}$decaying into charmless final state is at most $10^{-9}$. Thus, the triply bottom baryon can be only observed with a large amount of data in the future, such as the high luminosity LHC.

\section{DISCUSSIONS ON SU(3) SYMMETRY- BREAKING EFFECTS}

Our analysis of weak decays of triply heavy baryons heavily relies on the flavor $\mathrm{SU}(3)$ symmetry for light $u, d$, and $s$ quarks. At this stage, the $\mathrm{SU}(3)$ symmetry is helpful in figuring out the most promising decay channels toward the discovery. After the establishment of these states on the experimental side, it is valuable to use future experimental data to explore the flavor symmetry and the symmetrybreaking effects. 
TABLE XXII. Amplitudes for $\Omega_{c b b}$ decays into a bottom meson and a light baryon (decuplet).

\begin{tabular}{|c|c|c|c|}
\hline Channel & Amplitude & Channel & Amplitude \\
\hline$\Omega_{c b b}^{0} \rightarrow B^{-} \Delta^{+}$ & $\frac{2 d_{4} V_{u b} V_{c d}^{*}}{\sqrt{3}}$ & $\Omega_{c b b}^{0} \rightarrow \bar{B}^{0} \Sigma^{\prime 0}$ & $\sqrt{\frac{2}{3}} d_{4} V_{u b} V_{c s}^{*}$ \\
\hline$\Omega_{c b b}^{0} \rightarrow B^{-} \Sigma^{\prime+}$ & $\frac{2 d_{4} V_{u b} V_{c s}^{*}}{\sqrt{3}}$ & $\Omega_{c b b}^{0} \rightarrow \bar{B}_{s}^{0} \Sigma^{\prime 0}$ & $\sqrt{\frac{2}{3}} d_{4} V_{u b} V_{c d}^{*}$ \\
\hline$\Omega_{c b b}^{0} \rightarrow \bar{B}^{0} \Delta^{0}$ & $\frac{2 d_{4} V_{u b} V_{c d}^{*}}{\sqrt{3}}$ & $\Omega_{c b b}^{0} \rightarrow \bar{B}_{s}^{0} \Xi^{\prime 0}$ & $\frac{2 d_{4} V_{u b} V_{c s}^{*}}{\sqrt{3}}$ \\
\hline$\Omega_{c b b}^{0} \rightarrow B^{-} \Delta^{++} \pi^{-}$ & $\left(d_{1}+d_{7}\right) V_{u b} V_{c d}^{*}$ & $\Omega_{c b b}^{0} \rightarrow \bar{B}^{0} \Sigma^{\prime 0} K^{0}$ & $\frac{\left(d_{1}+2 d_{6}+d_{7}\right) V_{u b} V_{c d}^{*}}{\sqrt{6}}$ \\
\hline$\Omega_{c b b}^{0} \rightarrow B^{-} \Delta^{++} K^{-}$ & $\left(d_{1}+d_{7}\right) V_{u b} V_{c s}^{*}$ & $\Omega_{c b b}^{0} \rightarrow \bar{B}^{0} \Sigma^{\prime 0} \eta$ & $-\frac{1}{6}\left(3 d_{1}-2 d_{6}+d_{7}\right) V_{u b} V_{c s}^{*}$ \\
\hline$\Omega_{c b b}^{0} \rightarrow B^{-} \Delta^{+} \pi^{0}$ & $-\sqrt{\frac{2}{3}}\left(d_{1}-d_{6}\right) V_{u b} V_{c d}^{*}$ & $\Omega_{c b b}^{0} \rightarrow \bar{B}^{0} \Sigma^{\prime-} \pi^{+}$ & $\frac{\left(-d_{1}+d_{7}\right) V_{u b} V_{c s}^{*}}{\sqrt{3}}$ \\
\hline$\Omega_{c b b}^{0} \rightarrow B^{-} \Delta^{+} \bar{K}^{0}$ & $\frac{\left(d_{1}+d_{7}\right) V_{u b} V_{c s}^{*}}{\sqrt{3}}$ & $\Omega_{c b b}^{0} \rightarrow \bar{B}^{0} \Sigma^{\prime-} K^{+}$ & $\frac{\left(-d_{1}+d_{7}\right) V_{u b} V_{c d}^{*}}{\sqrt{3}}$ \\
\hline$\Omega_{c b b}^{0} \rightarrow B^{-} \Delta^{+} \eta$ & $\frac{1}{3} \sqrt{2}\left(d_{6}+d_{7}\right) V_{u b} V_{c d}^{*}$ & $\Omega_{c b b}^{0} \rightarrow \bar{B}^{0} \Xi^{\prime 0} K^{0}$ & $\frac{2 d_{6} V_{u b} V_{c s}^{*}}{\sqrt{3}}$ \\
\hline$\Omega_{c b b}^{0} \rightarrow B^{-} \Delta^{0} \pi^{+}$ & $\frac{\left(-d_{1}+2 d_{6}+d_{7}\right) V_{u b} V_{c d}^{*}}{\sqrt{3}}$ & $\Omega_{c b b}^{0} \rightarrow \bar{B}^{0} \Xi^{\prime-} K^{+}$ & $\frac{\left(-d_{1}+d_{7}\right) V_{u b} V_{c s}^{*}}{\sqrt{3}}$ \\
\hline$\Omega_{c b b}^{0} \rightarrow B^{-} \Sigma^{\prime+} \pi^{0}$ & $\frac{\left(-d_{1}+2 d_{6}+d_{7}\right) V_{u b} V_{c s}^{*}}{\sqrt{6}}$ & $\Omega_{c b b}^{0} \rightarrow \bar{B}_{s}^{0} \Delta^{+} K^{-}$ & $\frac{2 d_{6} V_{u b} V_{c d}^{*}}{\sqrt{3}}$ \\
\hline$\Omega_{c b b}^{0} \rightarrow B^{-} \Sigma^{\prime+} K^{0}$ & $\frac{\left(d_{1}+d_{7}\right) V_{u b} V_{c d}^{*}}{\sqrt{3}}$ & $\Omega_{c b b}^{0} \rightarrow \bar{B}_{s}^{0} \Delta^{0} \bar{K}^{0}$ & $\frac{2 d_{6} V_{u b} V_{c d}^{*}}{\sqrt{3}}$ \\
\hline$\Omega_{c b b}^{0} \rightarrow B^{-} \Sigma^{\prime+} \eta$ & $-\frac{\left(3 d_{1}-2 d_{6}+d_{7}\right) V_{u b} V_{c s}^{*}}{3 \sqrt{2}}$ & $\Omega_{c b b}^{0} \rightarrow \bar{B}_{s}^{0} \Sigma^{\prime+} \pi^{-}$ & $\frac{\left(d_{1}+d_{7}\right) V_{u b} V_{c d}^{*}}{\sqrt{3}}$ \\
\hline$\Omega_{c b b}^{0} \rightarrow B^{-} \Sigma^{\prime 0} \pi^{+}$ & $\frac{\left(-d_{1}+2 d_{6}+d_{7}\right) V_{u b} V_{c s}^{*}}{\sqrt{6}}$ & $\Omega_{c b b}^{0} \rightarrow \bar{B}_{s}^{0} \Sigma^{\prime+} K^{-}$ & $\frac{\left(d_{1}+2 d_{6}+d_{7}\right) V_{u b} V_{c s}^{*}}{\sqrt{3}}$ \\
\hline$\Omega_{c b b}^{0} \rightarrow B^{-} \Sigma^{\prime 0} K^{+}$ & $\frac{\left(-d_{1}+2 d_{6}+d_{7}\right) V_{u b} V_{c d}^{*}}{\sqrt{6}}$ & $\Omega_{c b b}^{0} \rightarrow \bar{B}_{s}^{0} \Sigma^{\prime 0} \pi^{0}$ & $-\frac{d_{1} V_{u b} V_{c d}^{*}}{\sqrt{3}}$ \\
\hline$\Omega_{c b b}^{0} \rightarrow B^{-} \Xi^{\prime 0} K^{+}$ & $\frac{\left(-d_{1}+2 d_{6}+d_{7}\right) V_{u b} V_{c s}^{*}}{\sqrt{3}}$ & $\Omega_{c b b}^{0} \rightarrow \bar{B}_{s}^{0} \Sigma^{\prime 0} \bar{K}^{0}$ & $\frac{\left(d_{1}+2 d_{6}+d_{7}\right) V_{u b} V_{c s}^{*}}{\sqrt{6}}$ \\
\hline$\Omega_{c b b}^{0} \rightarrow \bar{B}^{0} \Delta^{+} \pi^{-}$ & $\frac{\left(d_{1}+2 d_{6}+d_{7}\right) V_{u b} V_{c d}^{*}}{\sqrt{3}}$ & $\Omega_{c b b}^{0} \rightarrow \bar{B}_{s}^{0} \Sigma^{\prime 0} \eta$ & $\frac{1}{3}\left(-2 d_{6}+d_{7}\right) V_{u b} V_{c d}^{*}$ \\
\hline$\Omega_{c b b}^{0} \rightarrow \bar{B}^{0} \Delta^{+} K^{-}$ & $\frac{\left(d_{1}+d_{7}\right) V_{u b} V_{c s}^{*}}{\sqrt{3}}$ & $\Omega_{c b b}^{0} \rightarrow \bar{B}_{s}^{0} \Sigma^{\prime-} \pi^{+}$ & $\frac{\left(-d_{1}+d_{7}\right) V_{u b} V_{c d}^{*}}{\sqrt{3}}$ \\
\hline$\Omega_{c b b}^{0} \rightarrow \bar{B}^{0} \Delta^{0} \pi^{0}$ & $-\sqrt{\frac{2}{3}}\left(d_{1}+d_{6}\right) V_{u b} V_{c d}^{*}$ & $\Omega_{c b b}^{0} \rightarrow \bar{B}_{s}^{0} \Xi^{\prime 0} \pi^{0}$ & $\frac{\left(-d_{1}+d_{7}\right) V_{u b} V_{c s}^{*}}{\sqrt{6}}$ \\
\hline$\Omega_{c b b}^{0} \rightarrow \bar{B}^{0} \Delta^{0} \bar{K}^{0}$ & $\frac{\left(d_{1}+d_{7}\right) V_{u b} V_{c s}^{*}}{\sqrt{3}}$ & $\Omega_{c b b}^{0} \rightarrow \bar{B}_{s}^{0} \Xi^{\prime 0} K^{0}$ & $\frac{\left(d_{1}+d_{7}\right) V_{u b} V_{c d}^{*}}{\sqrt{3}}$ \\
\hline$\Omega_{c b b}^{0} \rightarrow \bar{B}^{0} \Delta^{0} \eta$ & $\frac{1}{3} \sqrt{2}\left(d_{6}+d_{7}\right) V_{u b} V_{c d}^{*}$ & $\Omega_{c b b}^{0} \rightarrow \bar{B}_{s}^{0} \Xi^{\prime 0} \eta$ & $-\frac{\left(3 d_{1}+4 d_{6}+d_{7}\right) V_{u b} V_{c s}^{*}}{3 \sqrt{2}}$ \\
\hline$\Omega_{c b b}^{0} \rightarrow \bar{B}^{0} \Delta^{-} \pi^{+}$ & $\left(-d_{1}+d_{7}\right) V_{u b} V_{c d}^{*}$ & $\Omega_{c b b}^{0} \rightarrow \bar{B}_{s}^{0} \Xi^{\prime-} \pi^{+}$ & $\frac{\left(-d_{1}+d_{7}\right) V_{u b} V_{c s}^{*}}{\sqrt{3}}$ \\
\hline$\Omega_{c b b}^{0} \rightarrow \bar{B}^{0} \Sigma^{\prime+} \pi^{-}$ & $\frac{2 d_{6} V_{u b} V_{c s}^{*}}{\sqrt{3}}$ & $\Omega_{c b b}^{0} \rightarrow \bar{B}_{s}^{0} \Xi^{\prime-} K^{+}$ & $\frac{\left(-d_{1}+d_{7}\right) V_{u b} V_{c d}^{*}}{\sqrt{3}}$ \\
\hline$\Omega_{c b b}^{0} \rightarrow \bar{B}^{0} \Sigma^{\prime 0} \pi^{0}$ & $-\frac{\left(d_{1}+2 d_{6}-d_{7}\right) V_{u b} V_{c s}^{*}}{2 \sqrt{3}}$ & $\Omega_{c b b}^{0} \rightarrow \bar{B}_{s}^{0} \Omega^{-} K^{+}$ & $\left(-d_{1}+d_{7}\right) V_{u b} V_{c s}^{*}$ \\
\hline
\end{tabular}

TABLE XXIII. Cabibbo-allowed decays of $\Omega_{c c c}$ with typical branching fractions at a few percent level. A light meson $\bar{K}^{0}$ can be replaced by a $\bar{K}^{* 0}$.

\begin{tabular}{lccc}
\hline \hline Channel & Channel & Channel & Channel \\
\hline$\Omega_{c c c}^{++} \rightarrow \Omega_{c c}^{+} \ell^{+} \nu_{\ell}$ & $\Omega_{c c c}^{++} \rightarrow \Xi_{c}^{\prime+} D^{0} \ell^{+} \nu_{\ell}$ & $\Omega_{c c c}^{++} \rightarrow \Xi_{c}^{0} D^{+} \ell^{+} \nu_{\ell}$ & $\Omega_{c c c}^{++} \rightarrow \Xi_{c c}^{+} \bar{K}^{0} \ell^{+} \nu_{\ell}$ \\
$\Omega_{c c c}^{++} \rightarrow \Xi_{c}^{+} D^{0} \ell^{+} \nu_{\ell}$ & $\Omega_{c c c}^{++} \rightarrow \Xi_{c}^{\prime 0} D^{+} \ell^{+} \nu_{\ell}$ & $\Omega_{c c c}^{++} \rightarrow \Xi_{c c}^{++} K^{-} \ell^{+} \nu_{\ell}$ & $\Omega_{c c c}^{++} \rightarrow \Omega_{c}^{0} D_{s}^{+} \ell^{+} \nu_{\ell}$ \\
$\Omega_{c c c}^{++} \rightarrow \Xi_{c c}^{++} \bar{K}^{0}$ & $\Omega_{c c c}^{++} \rightarrow \Omega_{c c}^{+} \pi^{+}$ & & \\
$\Omega_{c c c}^{++} \rightarrow \Xi_{c c}^{++} \bar{K}^{0} \pi^{0}$ & $\Omega_{c c c}^{++} \rightarrow \Xi_{c c}^{+} \pi^{+} \bar{K}^{0}$ & $\Omega_{c c c}^{++} \rightarrow \Xi_{c c}^{++} K^{-} \pi^{+}$ & $\Omega_{c c c}^{++} \rightarrow \Omega_{c c}^{+} K^{+} \bar{K}^{0}$ \\
$\Omega_{c c c}^{++} \rightarrow \Xi_{c}^{+} D^{+}$ & $\Omega_{c c c}^{++} \rightarrow \Xi_{c}^{\prime+} D^{+}$ & & \\
$\Omega_{c c c}^{++} \rightarrow \Lambda_{c}^{+} D^{+} \bar{K}^{0}$ & $\Omega_{c c c}^{++} \rightarrow \Xi_{c}^{+} D_{s}^{+} \bar{K}^{0}$ & $\Omega_{c c c}^{++} \rightarrow \Xi_{c}^{+} D^{+} \pi^{0}$ & $\Omega_{c c c}^{++} \rightarrow \Xi_{c}^{0} D^{+} \pi^{+}$ \\
$\Omega_{c c c}^{++} \rightarrow \Xi_{c}^{+} D^{0} \pi^{+}$ & & & \\
$\Omega_{c c c}^{++} \rightarrow \Sigma_{c}^{++} D^{0} \bar{K}^{0}$ & $\Omega_{c c c}^{++} \rightarrow \Xi_{c}^{\prime 0} D^{+} \pi^{+}$ & $\Omega_{c c c}^{++} \rightarrow \Xi_{c}^{\prime+} D^{0} \pi^{+}$ & $\Omega_{c c c}^{++} \rightarrow \Omega_{c}^{0} D^{+} K^{+}$ \\
$\Omega_{c c c}^{++} \rightarrow \Sigma_{c}^{++} D^{+} K^{-}$ & $\Omega_{c c c}^{++} \rightarrow \Xi_{c}^{\prime+} D_{s}^{+} \bar{K}^{0}$ & $\Omega_{c c c}^{++} \rightarrow \Xi_{c}^{++} D^{+} \pi^{0}$ & $\Omega_{c c c}^{++} \rightarrow \Omega_{c}^{0} D_{s}^{+} \pi^{+}$ \\
$\Omega_{c c c}^{++} \rightarrow \Sigma_{c}^{+} D^{+} \bar{K}^{0}$ & & & \\
\hline \hline
\end{tabular}


TABLE XXIV. CKM-allowed decay channels of $\Omega_{b b b}^{-}$.

\begin{tabular}{lccc}
\hline \hline Channel & Channel & Channel & Channel \\
\hline$\Omega_{b b b}^{-} \rightarrow \Xi_{b b}^{0} D^{0} \ell^{-} \bar{\nu}_{\ell}$ & $\Omega_{b b b}^{-} \rightarrow \Xi_{b c}^{+} B^{-} \ell^{-} \bar{\nu}_{\ell}$ & $\Omega_{b b b}^{-} \rightarrow \Omega_{b b}^{-} D_{s}^{+} \ell^{-} \bar{\nu}_{\ell}$ & $\Omega_{b b b}^{-} \rightarrow \Omega_{b c}^{0} \bar{B}_{s}^{0} \ell^{-} \bar{\nu}_{\ell}$ \\
$\Omega_{b b b}^{-} \rightarrow \Xi_{b b}^{-} D^{+} \ell^{-} \bar{\nu}_{\ell}$ & $\Omega_{b b b}^{-} \rightarrow \Xi_{b c}^{0} \bar{B}^{0} \ell^{-} \bar{\nu}_{\ell}$ & & \\
$\Omega_{b b b}^{-} \rightarrow \Omega_{b b}^{-} J / \psi$ & $\Omega_{b b b}^{-} \rightarrow \Xi_{b}^{\prime 0} B^{-} J / \psi$ & $\Omega_{b b b}^{-} \rightarrow \Xi_{b b}^{0} K^{-} J / \psi$ & $\Omega_{b b b}^{-} \rightarrow \Omega_{b}^{-} \bar{B}_{s}^{0} J / \psi$ \\
$\Omega_{b b b}^{-} \rightarrow \Xi_{b}^{0} B^{-} J / \psi$ & $\Omega_{b b b}^{-} \rightarrow \Xi_{b}^{\prime} \bar{B}^{0} J / \psi$ & $\Omega_{b b b}^{-} \rightarrow \Xi_{b b}^{-} \bar{K}^{0} J / \psi$ & $\Omega_{b b b}^{-} \rightarrow \Xi_{b}^{-} \bar{B}^{0} J / \psi$ \\
$\Omega_{b b b}^{-} \rightarrow \Omega_{b b c}^{0} D_{s}^{-}$ & $\Omega_{b b b}^{-} \rightarrow \Omega_{b b c}^{0} D^{-} \bar{K}^{0}$ & $\Omega_{b b b}^{-} \rightarrow \Omega_{b b c}^{0} \bar{D}^{0} K^{-}$ & \\
$\Omega_{b b b}^{-} \rightarrow \Omega_{b b c}^{0} \pi^{-}$ & $\Omega_{b b b}^{-} \rightarrow \Omega_{b b c}^{0} K^{0} K^{-}$ & & \\
$\Omega_{b b b}^{-} \rightarrow \Xi_{b b}^{-} D^{0}$ & $\Omega_{b b b}^{-} \rightarrow \Xi_{b b}^{-} D^{+} \pi^{-}$ & $\Omega_{b b b}^{-} \rightarrow \Xi_{b b}^{-} D^{0} \pi^{0}$ & $\Omega_{b b b}^{-} \rightarrow \Omega_{b b}^{-} D^{0} K^{0}$ \\
$\Omega_{b b b}^{-} \rightarrow \Lambda_{b}^{0} B^{-} D^{0}$ & $\Omega_{b b b}^{-} \rightarrow \Sigma_{b}^{-} B^{-} D^{+}$ & $\Omega_{b b b}^{-} \rightarrow \Xi_{b}^{-} \bar{B}_{s}^{0} D^{0}$ & $\Omega_{b b b}^{-} \rightarrow \Xi_{b}^{\prime} B^{-} D_{s}^{+}$ \\
$\Omega_{b b b}^{-} \rightarrow \Xi_{b b}^{0} D^{0} \pi^{-}$ & $\Omega_{b b b}^{-} \rightarrow \Sigma_{b}^{-} \bar{B}^{0} D^{0}$ & $\Omega_{b b b}^{-} \rightarrow \Omega_{b b}^{-} D_{s}^{+} \pi^{-}$ & $\Omega_{b b b}^{-} \rightarrow \Xi_{b}^{\prime-} \bar{B}_{s}^{0} D^{0}$ \\
$\Omega_{b b b}^{-} \rightarrow \Xi_{b}^{-} B^{-} D_{s}^{+}$ & $\Omega_{b b b}^{-} \rightarrow \Xi_{b b}^{-} D_{s}^{+} K^{-}$ & $\Omega_{b b b}^{-} \rightarrow \Sigma_{b}^{0} B^{-} D^{0}$ & \\
\hline \hline
\end{tabular}

In the Standard Model, symmetry-breaking effects arise from the unequal quark masses, $m_{s} \gg m_{u} \sim m_{d}$. In heavy bottom-quark decays, we expect the heavy-quark expansion might fairly work, and thus the symmetry-breaking effects emerging as power corrections $m_{u, d, s} / m_{b}$ might not be severe. Current studies on $B$ decays indicate the flavor symmetry-breaking effects are typically smaller than $20 \%$.

It is widely believed that the symmetry breaking in charm-quark decays could be sizable. In a recent analysis [61], the authors explored the breaking effects of the SU(3) flavor symmetry in singly Cabibbo-suppressed decays of charmed baryons, namely, $\Lambda_{c}$ and $\Xi_{c}$. We quote their results for the three amplitudes due to the $\left(H_{\overline{6}}\right)$ in the SU(3) limit,

$$
\begin{aligned}
& \left|a_{1}\right|=(0.252 \pm 0.005) \mathrm{GeV}^{3}, \\
& \left|a_{2}\right|=(0.127 \pm 0.009) \mathrm{GeV}^{3}, \\
& \left|a_{3}\right|=(0.091 \pm 0.015) \mathrm{GeV}^{3},
\end{aligned}
$$

while the $H_{15}$ contributions have been neglected. Instead, three amplitudes due to the symmetry breaking are incorporated and have been fitted as [61]

$$
\begin{aligned}
& \left|v_{1}\right|=(0.090 \pm 0.032) \mathrm{GeV}^{3}, \\
& \left|v_{2}\right|=(0.037 \pm 0.013) \mathrm{GeV}^{3}, \\
& \left|v_{3}\right|=(0.025 \pm 0.012) \mathrm{GeV}^{3}
\end{aligned}
$$

Compared to the three SU(3) amplitudes in Eq. (92), these symmetry-breaking terms are sizable but still smaller in magnitude. Thus, we expect that the relations derived in our analysis will not be completely ruined, and many of them can be precisely examined on the experimental side in the future.

\section{CONCLUSIONS}

Up to date, the quark model is a most successful theoretical tool to describe the hadron spectrum, especially the lowest-lying hadrons. Since the charm and bottom quarks are much heavier than the lighter ones, hadrons with a different number of heavy quarks will have distinct dynamics. On the experimental side, light hadrons with no heavy quark, singly heavy baryons, and doubly heavy baryons have been established, but triply heavy baryons are still missing. Thus, it deserves more theoretical and experimental effort to study various properties of triply heavy baryons from both the theoretical and experimental sides.

In this work, we have systematically analyzed weak decays of triply heavy baryons for the first time in the literature. Decay amplitudes for various transitions have been parametrized in terms of the SU(3)-independent amplitudes. Using these results, we find a number of relations for the partial decay widths. We also give a list of decay channels with sizable branching fractions. We suggest our experimental colleagues perform a search at hadron colliders and the electron and positron collisions in the future.

\section{ACKNOWLEDGMENTS}

The authors are grateful to Professor Ji-Bo He for useful discussions and valuable comments. We thank Zhen-Xing Zhao for checking the formulas of decay widths. W. W. is thankful for the hospitality from Professor C. Q. Geng and Professor Xiao-Gang $\mathrm{He}$ at National Center for Theoretical Sciences, Hsinchu, where this work was finalized. This work is supported in part by National Natural Science Foundation of China under Grants No. 11575110, No. 11655002, and No. 11735010 and Natural Science Foundation of Shanghai under Grant No. 15DZ2272100. 
[1] H. X. Chen, W. Chen, X. Liu, and S. L. Zhu, Phys. Rep. 639, 1 (2016).

[2] H. X. Chen, W. Chen, X. Liu, Y. R. Liu, and S. L. Zhu, Rep. Prog. Phys. 80, 076201 (2017).

[3] A. Ali, J. S. Lange, and S. Stone, Prog. Part. Nucl. Phys. 97, 123 (2017).

[4] F. K. Guo, C. Hanhart, U. G. Meiner, Q. Wang, Q. Zhao, and B. S. Zou, Rev. Mod. Phys. 90, 015004 (2018).

[5] R. Aaij et al. (LHCb Collaboration), Phys. Rev. Lett. 119, 112001 (2017).

[6] C. Patrignani et al. (Particle Data Group Collaboration), Chin. Phys. C 40, 100001 (2016).

[7] Y. Jia, J. High Energy Phys. 10 (2006) 073.

[8] A. P. Martynenko, Phys. Lett. B 663, 317 (2008).

[9] B. Patel, A. Majethiya, and P. C. Vinodkumar, Pramana 72, 679 (2009).

[10] J. R. Zhang and M. Q. Huang, Phys. Lett. B 674, 28 (2009).

[11] S. Meinel, Phys. Rev. D 82, 114514 (2010).

[12] F. J. Llanes-Estrada, O. I. Pavlova, and R. Williams, Eur. Phys. J. C 72, 2019 (2012).

[13] Z. G. Wang, Commun. Theor. Phys. 58, 723 (2012).

[14] S. Meinel, Phys. Rev. D 85, 114510 (2012).

[15] T. M. Aliev, K. Azizi, and M. Savci, J. High Energy Phys. 04 (2013) 042.

[16] M. Padmanath, R. G. Edwards, N. Mathur, and M. Peardon, Phys. Rev. D 90, 074504 (2014).

[17] Z. S. Brown, W. Detmold, S. Meinel, and K. Orginos, Phys. Rev. D 90, 094507 (2014).

[18] K. W. Wei, B. Chen, and X. H. Guo, Phys. Rev. D 92, 076008 (2015).

[19] K. W. Wei, B. Chen, N. Liu, Q. Q. Wang, and X. H. Guo, Phys. Rev. D 95, 116005 (2017).

[20] K. Thakkar, A. Majethiya, and P. C. Vinodkumar, Eur. Phys. J. Plus 131, 339 (2016).

[21] Y. Q. Chen and S. Z. Wu, J. High Energy Phys. 08 (2011) 144; 09 (2011) 89.

[22] M. A. Gomshi Nobary and R. Sepahvand, Phys. Rev. D 71, 034024 (2005).

[23] J. M. Flynn, E. Hernandez, and J. Nieves, Phys. Rev. D 85, 014012 (2012).

[24] C. Q. Geng, Y. K. Hsiao, C. W. Liu, and T. H. Tsai, J. High Energy Phys. 11 (2017) 147.

[25] D. Zeppenfeld, Z. Phys. C 8, 77 (1981).

[26] M. J. Savage and M. B. Wise, Phys. Rev. D 39, 3346 (1989); 40, 3127(E) (1989).

[27] M. Gronau, O. F. Hernandez, D. London, and J. L. Rosner, Phys. Rev. D 50, 4529 (1994).

[28] B. Grinstein and R.F. Lebed, Phys. Rev. D 53, 6344 (1996).

[29] X. G. He, Eur. Phys. J. C 9, 443 (1999).

[30] N. G. Deshpande, X. G. He, and J. Q. Shi, Phys. Rev. D 62 , 034018 (2000).

[31] X. G. He, J. Y. Leou, and C. Y. Wu, Phys. Rev. D 62, 114015 (2000).
[32] N. G. Deshpande and X. G. He, Phys. Rev. Lett. 75, 1703 (1995).

[33] X. G. He, Y. K. Hsiao, J. Q. Shi, Y. L. Wu, and Y. F. Zhou, Phys. Rev. D 64, 034002 (2001).

[34] H. K. Fu, X. G. He, Y. K. Hsiao, and J. Q. Shi, Chin. J. Phys. (Taipei) 41, 601 (2003).

[35] C. W. Chiang, M. Gronau, Z. Luo, J. L. Rosner, and D. A. Suprun, Phys. Rev. D 69, 034001 (2004).

[36] C. W. Chiang, M. Gronau, J. L. Rosner, and D. A. Suprun, Phys. Rev. D 70, 034020 (2004).

[37] C. W. Chiang and Y. F. Zhou, J. High Energy Phys. 12 (2006) 027.

[38] Y. Li, C. D. Lu, and W. Wang, Phys. Rev. D 77, 054001 (2008).

[39] W. Wang, Y. M. Wang, D. S. Yang, and C. D. Lu, Phys. Rev. D 78, 034011 (2008).

[40] C. W. Chiang and Y.F. Zhou, J. High Energy Phys. 03 (2009) 055.

[41] H. Y. Cheng, C. W. Chiang, and A. L. Kuo, Phys. Rev. D 91, 014011 (2015).

[42] X. G. He, G. N. Li, and D. Xu, Phys. Rev. D 91, 014029 (2015).

[43] X. G. He and G. N. Li, Phys. Lett. B 750, 82 (2015).

[44] Y. K. Hsiao, C. F. Chang, and X. G. He, Phys. Rev. D 93, 114002 (2016).

[45] X. G. He, W. Wang, and R. L. Zhu, J. Phys. G 44, 014003 (2017).

[46] X. G. He, S. F. Li, B. Ren, and X. B. Yuan, Phys. Rev. D 96, 053004 (2017).

[47] C.-D. Lü, W. Wang, and F. S. Yu, Phys. Rev. D 93, 056008 (2016).

[48] H. Y. Cheng, C. W. Chiang, and A. L. Kuo, Phys. Rev. D 93, 114010 (2016).

[49] H. Y. Cheng and C. W. Chiang, Phys. Rev. D 86, 014014 (2012).

[50] H. Y. Cheng and S. Oh, J. High Energy Phys. 09 (2011) 024.

[51] W. Wang, Z. P. Xing, and J. Xu, Eur. Phys. J. C 77, 800 (2017).

[52] Y. J. Shi, W. Wang, Y. Xing, and J. Xu, Eur. Phys. J. C 78, 56 (2018).

[53] C. Q. Geng, Y. K. Hsiao, Y. H. Lin, and L. L. Liu, Phys. Lett. B 776, 265 (2018).

[54] C. Q. Geng, Y. K. Hsiao, C. W. Liu, and T.H. Tsai, arXiv:1801.03276.

[55] X. G. He and W. Wang, arXiv:1803.04227.

[56] A. Lenz, Int. J. Mod. Phys. A 30, 1543005 (2015).

[57] F. Krinner, A. Lenz, and T. Rauh, Nucl. Phys. B876, 31 (2013).

[58] W. Wang, Y. L. Shen, and C. D. Lu, Phys. Rev. D 79, 054012 (2009).

[59] Z. G. Wang, Eur. Phys. J. A 45, 267 (2010).

[60] M. Gronau, Phys. Lett. B 492, 297 (2000).

[61] C. Q. Geng, Y. K. Hsiao, C. W. Liu, and T.H. Tsai, arXiv:1804.01666. 